This item was submitted to Loughborough's Research Repository by the author.

Items in Figshare are protected by copyright, with all rights reserved, unless otherwise indicated.

\title{
Local normal forms for c-projectively equivalent metrics and proof of the Yano-Obata conjecture in arbitrary signature. Proof of the projective Lichnerowicz conjecture for Lorentzian metrics
}

\section{PLEASE CITE THE PUBLISHED VERSION}

http://arxiv.org/abs/1510.00275

\section{PUBLISHER}

arXiv.org

VERSION

SMUR (Submitted Manuscript Under Review)

\section{PUBLISHER STATEMENT}

This work is made available according to the conditions of the Creative Commons Attribution-NonCommercialNoDerivatives 4.0 International (CC BY-NC-ND 4.0) licence. Full details of this licence are available at: https://creativecommons.org/licenses/by-nc-nd/4.0/

\section{LICENCE}

CC BY-NC-ND 4.0

\section{REPOSITORY RECORD}

Bolsinov, Alexey V., Vladimir S. Matveev, and Steffan Rosemann. 2019. "Local Normal Forms for Cprojectively Equivalent Metrics and Proof of the Yano-obata Conjecture in Arbitrary Signature. Proof of the Projective Lichnerowicz Conjecture for Lorentzian Metrics". figshare. https://hdl.handle.net/2134/19554. 


\title{
LOCAL NORMAL FORMS FOR C-PROJECTIVELY EQUIVALENT METRICS AND PROOF OF THE YANO-OBATA CONJECTURE IN ARBITRARY SIGNATURE. PROOF OF THE PROJECTIVE LICHNEROWICZ CONJECTURE FOR LORENTZIAN METRICS
}

\author{
ALEXEY V. BOLSINOV, VLADIMIR S. MATVEEV, AND STEFAN ROSEMANN
}

\begin{abstract}
Two Kähler metrics on a complex manifold are called c-projectively equivalent if their $J$-planar curves coincide. These curves are defined by the property that the acceleration is complex proportional to the velocity. We give an explicit local description of all pairs of c-projectively equivalent Kähler metrics of arbitrary signature and use this description to prove the classical Yano-Obata conjecture: we show that on a closed connected Kähler manifold of arbitrary signature, any c-projective vector field is an affine vector field unless the manifold is $\mathbb{C} P^{n}$ with (a multiple of) the Fubini-Study metric. As a by-product, we prove the projective Lichnerowicz conjecture for metrics of Lorentzian signature: we show that on a closed connected Lorentzian manifold, any projective vector field is an affine vector field.
\end{abstract}

\section{CONTEnTs}

1. Introduction 2

1.1. Definitions and description of results 2

1.2. Main idea $\quad 4$

1.3. Local description of c-projectively equivalent metrics 5

1.4. Structure of the paper 11

2. Canonical Killing vector fields for c-projectively equivalent metrics 12

3. Reduction to the real projective setting $\quad 16$

3.1. The Kähler quotient w.r.t. a local isometric hamiltonian $\mathbb{R}^{\ell}$-action 16

3.2. Reduction from the c-projective to the projective setting 19

4. Local description of c-projectively equivalent metrics $r$

4.1. Local description of the quotients of c-projectively equivalent metrics and lifting 19

4.2. Realisation 22

4.3. Explicit formulas 26

5. Proof of the Yano-Obata conjecture (Theorem 1.1) 28

5.1. Conventions and degree of mobility 29

5.2. Scheme of the proof $\quad 29$

5.3. C-projectively invariant form of equation (1.4) and special features of $D(g, J)=2 \quad 30$

5.4. Properties of the eigenvalues of $A \quad 31$

5.5. Explicit formulas for the non-constant block 33

5.6. There is only one non-constant eigenvalue $\quad 37$

5.7. Proof of Theorem 1.1 when there is only one non-constant eigenvalue 41

6. Final step of the proof of Theorem 1.2: the case of a non-constant Jordan block 45

Appendix A. Eigenvalues of the curvature operator $\quad 52$

$\begin{array}{ll}\text { References } & 55\end{array}$ 


\section{INTRODUCTION}

1.1. Definitions and description of results. Let $(M, g, J)$ be a Kähler manifold of arbitrary signature of real dimension $2 n \geq 4$. We denote by $\nabla$ the Levi-Civita connection of $g$ and let $\omega=g(J \cdot, \cdot)$ denote the Kähler form. All objects under consideration are assumed to be sufficiently smooth.

A regular curve $\gamma: \mathbb{R} \supseteq I \rightarrow M$ is called $J$-planar if there exist functions $\alpha, \beta: I \rightarrow \mathbb{R}$ such that

$$
\nabla_{\dot{\gamma}(t)} \dot{\gamma}(t)=\alpha \dot{\gamma}(t)+\beta J(\dot{\gamma}(t)) \text { for all } t \in I,
$$

where $\dot{\gamma}=\frac{\mathrm{d}}{\mathrm{d} t} \gamma$.

From the definition we see immediately that the property of $J$-planarity is independent of the parameterisation of the curve, and that geodesics are $J$-planar curves. We also see that $J$-planar curves form a much bigger family than the family of geodesics; at every point and in every direction there exist infinitely many geometrically different $J$-planar curves.

Two metrics $g$ and $\hat{g}$ of arbitrary signature that are Kähler w.r.t the same complex structure $J$ are c-projectively equivalent if any $J$-planar curve of $g$ is a $J$-planar curve of $\hat{g}$. Actually, the condition that the metrics are Kähler with respect to the same complex structure is not essential; it is an easy exercise to show that if any $J$-planar curve of a Kähler structure $(g, J)$ is a $\hat{J}$-planar curve of another Kähler structure $(\hat{g}, \hat{J})$, then $\hat{J}= \pm J$.

C-projective equivalence was introduced (under the name "h-projective equivalence") by Otsuki and Tashiro in $[35,40]$. Their motivation was to generalise the notion of projective equivalence to the Kähler situation. Since the notion of projective equivalence plays an essential role in our paper let us recall it. Two metrics $g$ and $\hat{g}$ of arbitrary signature are projectively equivalent, if each $g$-geodesic is, up to an appropriate reparameterisation, a $\hat{g}$-geodesic.

Otsuki and Tashiro have shown that projective equivalence is not interesting in the Kähler situation, since only simple examples are possible, and suggested c-projective equivalence as an interesting object of study instead. This suggestion appeared to be very fruitful and between the 1960s and the 1970s, the theory of c-projectively equivalent metrics and c-projective transformations was one of the main research topics in Japanese and Soviet (mostly Odessa and Kazan) differential geometry schools. For a collection of results of these times, see for example the survey [32] with more than 150 references. Moreover, two classical books [39, 43] contain chapters on c-projectively equivalent metrics and connections.

Relatively recently c-projective equivalence was re-introduced, under different names and because of different motivation. In fact, c-projectively equivalent metrics are essentially the same as hamiltonian 2-forms, defined and investigated in Apostolov et al. [1, 2, 3, 4]. Though the definition of hamiltonian 2-forms is visually different from that of c-projectively equivalent metrics, the defining equation [1, equation (12)] of a hamiltonian 2-form is algebraically equivalent to a reformulation (see (1.4) below) of the condition " $\hat{g}$ is c-projectively equivalent to $g$ " into the language of PDE. The motivation of Apostolov et al. to study hamiltonian 2-forms is different from that of Otsuki and Tashiro. Roughly speaking, in [1,2] Apostolov et al. observe that many interesting problems in Kähler geometry lead to hamiltonian 2-forms and suggest studying them. The motivation is justified in $[3,4]$, where the authors indeed construct interesting and useful examples of Kähler manifolds. In dimension $\geq 6$, c-projectively equivalent metrics are also essentially the same as hermitian conformal Killing (or twistor) $(1,1)$-forms studied in [33, 37, 38], see $[1$, Appendix A] or $[30, \S 1.3]$ for details. Finally, such metrics are closely related to the so-called Kähler-Liouville integrable systems of type $A$ introduced by Kiyohara et al. in [23].

Most recent interest in c-projectively equivalent metrics is due to the observation that one can study them with the help of so-called parabolic geometry. We refer to [14] for details. Certain non-trivial applications of the methods of parabolic geometry in the theory of c-projective transformations are in [28].

Our paper contains three main results. The first result is a local description (near a generic point) of c-projectively equivalent Kähler metrics of arbitrary signature. If $g$ is positive definite, such a description follows from the local description of hamiltonian 2-forms due to Apostolov 
et al. [1]. Although the precise statements are slightly lengthy, we indeed provide an explicit description of the components of the metrics and of the Kähler form $\omega=g(J \cdot, \cdot)$. The parameters in this description are almost arbitrary numbers and functions of one variable and, in certain cases, almost arbitrary affinely equivalent Kähler metrics of smaller dimension (note that the description of affinely equivalent Kähler metrics was recently obtained by Boubel in [13]).

It is hard to overestimate the future role of a local description in the local and global theory of c-projectively equivalent metrics. Almost all known local results can easily be proved using it. Roughly speaking, using the local description, one can reduce any problem that can be stated using geometric PDEs (for example, any problem involving the curvature) to the analysis of a system of ODEs. As we mentioned above, in the positive definite case, the description of c-projectively equivalent metrics in the language of hamiltonian 2-forms is due to Apostolov et al. [1], and with the help of such a description they did a lot. In particular they described possible topologies of closed manifolds admitting c-projectively equivalent metrics and constructed new examples of Einstein and extremal metrics on closed manifolds, see $[2,3,4]$. We expect similar applications of our description as well and, in particular, plan to study the topology of c-projectively equivalent closed Kähler manifolds of arbitrary signature in further papers.

A demonstration of the importance of the local description is our second main result, which is a proof of the natural generalisation of the Yano-Obata conjecture for Kähler manifolds of arbitrary signature. A vector field on a Kähler manifold is called c-projective if its local flow sends $J$-planar curves to $J$-planar curves, and affine if its local flow preserves the Levi-Civita connection.

Theorem 1.1 (Yano-Obata conjecture). Let $(M, g, J)$ be a closed connected Kähler manifold of arbitrary signature and of real dimension $2 n \geq 4$ such that it admits a c-projective vector field that is not an affine vector field. Then the manifold is isometric to $\left(\mathbb{C} P^{n}, c \cdot g_{F S}, J_{\text {standard }}\right)$ for some non-zero constant $c$, where $g_{F S}$ is the Fubini-Study metric.

For positive definite metrics, Theorem 1.1 was proved in [31], where also a history including a list of previously proven special cases can be found. The 4-dimensional version of Theorem 1.1 was recently proved in [10].

We see that a closed Kähler manifold with a non-affine c-projective vector field has definite signature. This phenomenon is, of course, essentially global since locally we can construct counterexamples in any signature. In dimension 4, such examples are described in [10], and in Proposition 5.7 we explicitly construct Kähler metrics of any dimension and any signature admitting non-affine cprojective vector fields. Let us also mention (see, e.g. [31, Example 2]) that $\left(\mathbb{C} P^{n}, c \cdot g_{F S}, J_{\text {standard }}\right.$ ) admits many non-affine c-projective vector fields.

As a by-product of our proof of the Yano-Obata conjecture (we explain in the next section why it is a by-product), we prove the possibly more popular projective Lichnerowicz conjecture for metrics of Lorentzian signature. Recall that a vector field is projective with respect to a (arbitrary, not necessarily Kähler) metric $g$, if its local flow sends geodesics viewed as unparameterised curves to geodesics.

Theorem 1.2 (Projective Lichnerowicz conjecture for metrics of Lorentzian signature). Let $(M, g)$ be a closed connected Lorentzian manifold of dimension $n \geq 2$. Then any projective vector field on $M$ is an affine vector field.

For Riemannian metrics, the analogue of Theorem 1.2 was proved in [25] (dimension 2) and [26] (dimension greater that 2 - this paper also contains a historical overview and a list of previously known special cases), see also [45]. In Japanese mathematics, this statement, at least in the Riemannian setting, is also known as projective Obata conjecture and was published many times as an important conjecture. For 2-dimensional Lorentzian manifolds, Theorem 1.2 was proved in [29].

We would like to emphasize here that our proofs of the Yano-Obata and Lichnerowicz conjectures are not generalisations of the proofs from [25, 26, 29, 31, 45], and are based on a different circle of ideas. In general, it is difficult to extend global statements about Riemannian metrics to the pseudo-Riemannian setting, since many "global" methods require definiteness of the metrics. This is also the case in our situation; the main ingredients of the proofs of $[26,25,31,45]$ are 
the global ordering of the eigenvalues of the endomorphisms $A$ and $L$ (given by (1.2) and (1.6) these endomorphisms play an important role in our paper), and an investigation of the behaviour of the sectional and holomorphic sectional curvature along the integral curves of the projective and c-projective vector fields (in $[26,31]$ it is shown that these curvatures are either constant or unbounded). Both ingredients do not exist in the case of indefinite signature; examples show that the eigenvalues of $A$ (resp. $L$ ) are not globally ordered anymore, and the unboundedness of the sectional curvature does not lead to any contradiction, since for metrics of indefinite signature the sectional or holomorphic sectional curvature is either constant or unbounded. Moreover, as follows from our calculations in $\S 5.6$, in the indefinite case, all curvature invariants along the integral curves of the projective and c-projective vector fields can be bounded. Other proofs of special cases of the Yano-Obata and Lichnerowicz conjecture (see e.g. [42, 44]) are based on the Bochner technique, which also requires that the metric is definite.

1.2. Main idea. The local description of c-projectively equivalent metrics will be given in Theorem 1.6 in $\S 1.3$ (which does not require this paragraph so a hurried reader can directly go there). The goal of this section is to explain the main idea of our solution. We hope that this allows the reader to see the geometry behind the formulas and also may be used in many other problems related to c-projectively equivalent metrics.

Experts always expected that projectively equivalent metrics must have a close relation with c-projectively equivalent metrics. The expectation is based on the following informal observation: most mathematicians that studied c-projectively equivalent metrics and c-projective vector fields studied projectively equivalent metrics and projective vector fields before. It appears that many ideas and many results in the theory of projectively equivalent metrics have their counterparts in the c-projective setting, though most of the proofs in the c-projective setting are longer and are more involved than their projective analogues. ${ }^{1}$

We suggest an explanation why the theories are closely related, which is simultaneously the main idea of our description. The following observation, which we formalise (and give a selfcontained proof) in $\S 2$, is crucial: c-projectively, but not affinely equivalent metrics $g$ and $\hat{g}$ allow us to construct vector fields $K_{1}, \ldots, K_{\ell}$ which preserve the complex structure and which are Killing with respect to both metrics. For hamiltonian 2-forms (at least for a positive definite metric), the existence of these Killing vector fields was shown by Apostolov et al. [1], and in the framework of Kähler-Liouville manifolds (under certain non-degeneracy assumptions) their existence was observed by Kiyohara and Topalov [23].

We consider the local action of these vector fields and the local quotient $Q$ of $M$ with respect to this action (it will be shown that such a quotient is well-defined near a generic point). Let us denote the quotient metrics by $g_{Q}$ and $\hat{g}_{Q}$. Notice that $Q$ is not a Kähler quotient and the metrics $g_{Q}$ and $\hat{g}_{Q}$ are in general not Kähler.

Main Observation. The following statements hold:

(1) $g_{Q}$ and $\hat{g}_{Q}$ are projectively equivalent;

(2) the metrics $g$ and $\hat{g}$ can be reconstructed from $g_{Q}$ and $\hat{g}_{Q}$ in a relatively straightforward way.

Recently, projectively equivalent metrics have been explicitly locally described in [9]. We obtain our description of c-projectively equivalent metrics by taking the formulas from [9] for the quotient metrics $g_{Q}$ and $\hat{g}_{Q}$ and then "reconstructing" $g$ and $\hat{g}$.

However, not every pair of projectively equivalent metrics $g_{Q}, \hat{g}_{Q}$ as considered in [9] can be obtained from a pair $g, \hat{g}$ of c-projectively equivalent metrics: we will describe the conditions that $g_{Q}$ and $\hat{g}_{Q}$ have to satisfy in order to arise as quotients from c-projectively equivalent metrics. These additional conditions actually simplify the formulas for the metrics $g_{Q}, \hat{g}_{Q}$ as compared to the formulas from [9] for the general case. Moreover, we show, assuming these conditions are

\footnotetext{
${ }^{1}$ This analogy between c-projective and projective geometry fails on the level of affine connections (note that the definition of c-projective equivalence makes also sense for affine connections which are not necessarily Levi-Civita connections): though both affine projective and affine c-projective geometries are parabolic geometries, there are essential differences between these theories if only connections are involved, see e.g. [14].
} 
satisfied, how to effectively reconstruct the initial metrics $g$ and $\hat{g}$. This yields our description of c-projectively equivalent Kähler metrics.

The relation between projectively and c-projectively equivalent metrics plays also an important role in the proof of the Yano-Obata conjecture. We will see that under the additional assumption that the degree of mobility is 2 (which means that the "space of c-projectively equivalent metrics" is two-dimensional - the formal definition is in $\S 5.1$ where it is also explained why it is the most non-trivial case in the proof of the Yano-Obata conjecture), a c-projective vector field on the initial manifold reduces to a projective vector field on the quotient.

We expect further applications of this observation which suggests, in the metric setting, an almost algorithmic way to produce results in c-projective geometry from results in projective geometry and the latter is much better developed.

Unfortunately, this almost algorithmic way does not automatically work in the other (c-projective $\rightarrow$ projective) direction. The reason is that the quotient metrics $g_{Q}$ and $\hat{g}_{Q}$, as already noticed, satisfy certain additional conditions. The most important of them is as follows: for the metrics $h=g_{Q}$ and $\hat{h}=\hat{g}_{Q}$ the endomorphism $L$ given by (1.6) below has no Jordan blocks with nonconstant eigevalues. For general projectively equivalent metrics, $L$ may have non-trivial Jordan blocks with nonconstant eigevalues. This is the only reason why we can not modify the proof of the Yano-Obata conjecture to obtain the proof of the projective Lichnerowicz conjectures for metrics of all signatures. For the metrics of Lorentzian signature, at most one non-trivial Jordan block may occur and after some additional work in $\S 6$ we exclude this case in the proof of the projective Lichnerowicz conjecture. The rest of the proof of the projective Lichnerowicz conjecture is a straightforward modification (actually, a simplification) of the proof of the Yano-Obata conjecture and when proving the projective Lichnerowicz conjecture in the "no-Jordan-blocks" case (Theorem 5.1), we confine ourselves with a series of remarks explaining necessary amendments.

1.3. Local description of c-projectively equivalent metrics. Let $(M, g, J)$ be a Kähler manifold of real dimension $2 n \geq 4$ and let $\nabla$ and $\omega=g(J \cdot, \cdot)$ denote the Levi-Civita connection and Kähler form respectively. We do not require that $g$ or any other Kähler metric that appears has positive signature.

Instead of the pair $(g, \hat{g})$ of c-projectively equivalent metrics it is appropriate to consider the pair $(g, A)$, where $A: T M \rightarrow T M$ is a hermitian (i.e. $g$-selfadjoint and $J$-commuting) endomorphism constructed from $g$ and $\hat{g}$ by

$$
A=A(g, \hat{g})=\left(\frac{\operatorname{det} \hat{g}}{\operatorname{det} g}\right)^{\frac{1}{2(n+1)}} \hat{g}^{-1} g .
$$

In this formula, we view $g, \hat{g}: T M \rightarrow T^{*} M$ as bundle isomorphisms. In tensor notation (with summation convention in force),

$$
A_{j}^{i}=\left(\frac{\operatorname{det} \hat{g}}{\operatorname{det} g}\right)^{\frac{1}{2(n+1)}} \hat{g}^{i k} g_{k j},
$$

where $\hat{g}^{i j}$ denotes the inverse to $\hat{g}_{i j}$, i.e. $\hat{g}^{i k} \hat{g}_{k j}=\delta_{j}^{i}$.

Clearly, one can reconstruct $\hat{g}$ from the pair $(g, A)$ and obtains

$$
\hat{g}=(\operatorname{det} A)^{-\frac{1}{2}} g\left(A^{-1} \cdot, \cdot\right) .
$$

The endomorphism $A$, introduced in [17], plays an important role in the theory of c-projectively equivalent metrics. One of the reasons for this is that the condition that $g$ and $\hat{g}$ are c-projectively equivalent amounts to the fact that the tensor $A$ satisfies the linear partial differential equation

$$
\nabla_{X} A=X^{b} \otimes \Lambda+\Lambda^{b} \otimes X+(J X)^{b} \otimes J \Lambda+(J \Lambda)^{b} \otimes J X,
$$

for all $X \in T M$, where $\Lambda=\frac{1}{4} \operatorname{grad}(\operatorname{tr} A)$ and $X^{b}=g(X, \cdot)$. We say that $g$ and $A$ are compatible in the c-projective sense or just c-compatible if $A$ is a hermitian endomorphism solving (1.4). In particular, any hermitian endomorphism $A$ with nowhere vanishing determinant and c-compatible with $g$ gives us a c-projectively equivalent metric $\hat{g}$ by (1.3), this metric is automatically Kähler with respect to $J$. 
Another reason for working with $A$ instead of $\hat{g}$ is that in our local description, the formulas for $(g, A)$ are much simpler than those for $(g, \hat{g})$.

We describe locally all Kähler structures $(g, J, \omega)$ admitting solutions $A$ to (1.4) in Theorem 1.6 below. Since the description is relatively complicated, we first consider two special cases corresponding to the "weakest" (Theorem 1.3) and "strongest" (Theorem 1.4) case of c-projective equivalence.

Note that any parallel hermitian endomorphism $A$ (i.e., satisfying $\nabla A=0$ ), in particular the identity Id : $T M \rightarrow T M$, is a solution to (1.4). Such solutions correspond to Kähler metrics $\hat{g}$ which are affinely equivalent to $g$, i.e., which have the same Levi-Civita connection as $g$.

Theorem 1.3 (Well-known special case of Theorem 1.6). Let $(M, g, J)$ be a Kähler manifold of arbitrary signature and $A: T M \rightarrow T M$ a parallel hermitian endomorphism. Then locally $(M, g, J)$ is a direct product of Kähler manifolds $\left(M_{\gamma}, g_{\gamma}, J_{\gamma}\right), \gamma=1, \ldots, N$, and $A$ decomposes as $A=A_{1}+\cdots+A_{N}$, where $A_{\gamma}: T M_{\gamma} \rightarrow T M_{\gamma}$ is a parallel hermitian endomorphism on $\left(M_{\gamma}, g_{\gamma}, J_{\gamma}\right)$ having either a single real eigenvalue $c_{\gamma}$ or a pair of complex-conjugate eigenvalues $c_{\gamma}, \bar{c}_{\gamma}$.

The above theorem is just the de Rham-Wu decomposition [15, 41] of the Kähler manifold into components corresponding to the parallel distributions given by the generalised eigenspaces of $A$. This is not a complete description of pairs $((g, J), A)$, where $(g, J)$ is Kähler and $A$ is a parallel hermitian endomorphism: what is left is an explicit description of the blocks $\left(g_{\gamma}, J_{\gamma}\right)$ and $A_{\gamma}$. In the positive definite case, the description of these blocks is trivial since in this case $A_{\gamma}$ is a constant multiple of Id : $T M_{\gamma} \rightarrow T M_{\gamma}$. If the signature of $g_{\gamma}$ is arbitrary, the local description of $\left(g_{\gamma}, J_{\gamma}\right)$ and $A_{\gamma}$ has recently been obtained by C. Boubel in [13]. Boubel's description of $\left(g_{\gamma}, J_{\gamma}, A_{\gamma}\right)$ is quite complicated, we will not repeat it here and refer to [13] for more details.

Remark 1.1. Let us reformulate the statement from Theorem 1.3 in matrix notation: we can find local coordinates such that the matrices of $g, J$ and $A$ in these coordinates are block-diagonal with the same structure of blocks:

$$
g=\left(\begin{array}{ccc}
g_{1} & & \\
& \ddots & \\
& & g_{N}
\end{array}\right), \quad J=\left(\begin{array}{ccc}
J_{1} & & \\
& \ddots & \\
& & J_{N}
\end{array}\right), \quad A=\left(\begin{array}{ccc}
A_{1} & & \\
& \ddots & \\
& & A_{N}
\end{array}\right) .
$$

In all matrices, the components of each block only depend on the corresponding coordinates and for each $\gamma=1, \ldots, N$ the endomorphism $A_{\gamma}$ is hermitian and parallel w.r.t. the Kähler structure $\left(g_{\gamma}, J_{\gamma}\right)$.

The "main idea" and "main observation" described in $\S 1.2$ become vacuous in the setting of Theorem 1.3: the number of "canonical" Killing vector fields $K_{1}, \ldots, K_{\ell}$ is zero, hence, the quotient of the manifold is the manifold itself. The "main observation" remains, of course, formally true but in this case projective equivalence is affine equivalence.

A special feature of the situation described in Theorem 1.3 is that the eigenvalues of $A$ are constant, and may have high multiplicities. Let us now consider the "strongest" special case of c-projective equivalence: all eigenvalues of $A$ are non-constant (when considered as functions on $M)$, and their multiplicity is minimal possible.

Consider two projectively equivalent pseudo-Riemannian metrics $h$ and $\hat{h}$ (i.e., metrics having the same unparametrised geodesics) and define the endomorphism $L$ by

$$
L=L(h, \hat{h})=\left|\frac{\operatorname{det} \hat{h}}{\operatorname{det} h}\right|^{\frac{1}{n+1}} \hat{h}^{-1} h .
$$

It is well known that $L$ satisfies the equation

$$
\nabla_{X} L=X^{b} \otimes \Lambda+\Lambda^{b} \otimes X, \quad \text { for all } X \in T M,
$$

where $\Lambda=\frac{1}{2} \operatorname{grad}(\operatorname{tr} L), X^{\mathrm{b}}=h(X, \cdot)$ and $\nabla$ denotes the Levi-Civita connection of $h$. Moreover, if $L$ is $h$-selfadjoint and non-degenerate, then (1.7) is equivalent to the projective equivalence of $h$ 
and $\hat{h}$ given by

$$
\hat{h}=|\operatorname{det} L|^{-1} h\left(L^{-1} \cdot, \cdot\right)
$$

see [39] and e.g. [8]. To emphasize both the difference and similarity with c-compatibility introduced above, we will say that $h$ and an $h$-selfadjoint endomorphism $L$ satisfying (1.7) are compatible in the projective sense or just compatible.

Example 1. Assume that on a certain domain $U \subset \mathbb{R}^{\ell}$ we have a compatible pair $h$ and $L$ for which the following conditions hold:

A1. The eigenvalues $\rho_{1}, \ldots, \rho_{\ell}$ of $L$ are all distinct at each point of $U$ (complex conjugate pairs $\rho, \bar{\rho}$ with $\operatorname{Im} \rho \neq 0$ are allowed too), which allows us to view them as smooth functions on $U$

A2. $\mathrm{d} \rho_{i} \neq 0$ at each point of $U, i=1, \ldots, \ell$.

We now explain how, starting from such a compatible pair

$$
h=\sum_{i, j=1}^{\ell} B_{i j}(x) \mathrm{d} x_{i} \mathrm{~d} x_{j} \quad \text { and } \quad L=\sum_{i, j=1}^{\ell} L_{j}^{i} \mathrm{~d} x_{j} \otimes \frac{\partial}{\partial x_{i}},
$$

one can naturally construct a c-compatible pair, i.e., a Kähler structure $(g, J, \omega)$ and a hermitian endomorphism $A$ satisfying (1.4). By $\mu_{1}, \ldots, \mu_{\ell}$ we denote the elementary symmetric polynomials in $\rho_{1}, \ldots, \rho_{\ell}$ (i.e., $\left(\tau+\rho_{1}\right) \ldots\left(\tau+\rho_{\ell}\right)=\tau^{\ell}+\mu_{1} \tau^{\ell-1}+\ldots+\mu^{\ell}$.) Notice that under the assumption that $\rho_{i}$ are all distinct and $\mathrm{d} \rho_{i} \neq 0$, both systems of functions $\rho_{i}$ 's and $\mu_{i}$ 's can be considered as local coordinates on $U$.

Consider a domain $V \subset \mathbb{R}^{\ell}$ with local coordinates $t_{1}, \ldots, t_{\ell}$ and define $g, \omega$ on $V \times U$ in the following way

$$
\begin{aligned}
g & =\sum_{\alpha, \beta=1}^{\ell} H_{\alpha \beta}(x) \mathrm{d} t_{\alpha} \mathrm{d} t_{\beta}+\sum_{i, j=1}^{\ell} B_{i j}(x) \mathrm{d} x_{i} \mathrm{~d} x_{j}, \\
\omega & =\sum_{\alpha=1}^{\ell} \mathrm{d} \mu_{\alpha} \wedge \mathrm{d} t_{\alpha},
\end{aligned}
$$

where $H_{\alpha \beta}=\sum_{i j} B^{i j}(x) \frac{\partial \mu_{\alpha}}{\partial x_{i}} \frac{\partial \mu_{\beta}}{\partial x_{j}}$ and $B^{i j}$ are the components of the matrix inverse to $B_{i j}$, i.e., $\sum_{k} B_{i k} B^{k j}=\delta_{i}^{j}$. We also set

$$
A=\sum_{\alpha, \beta=1}^{\ell} M_{\alpha}^{\beta}(x) \mathrm{d} t_{\beta} \otimes \frac{\partial}{\partial t_{\alpha}}+\sum_{i, j=1}^{\ell} L_{j}^{i}(x) \mathrm{d} x_{j} \otimes \frac{\partial}{\partial x_{i}},
$$

where $M_{\alpha}^{\beta}=\sum_{i, j} L_{j}^{i} \frac{\partial \mu_{\beta}}{\partial x_{i}} \frac{\partial x_{j}}{\partial \mu_{\alpha}}$.

Equivalently, in matrix form w.r.t. the coordinates $t_{1}, \ldots, t_{\ell}, x_{1}, \ldots, x_{\ell}$, the above expressions take the form

$$
g=\left(\begin{array}{cc}
P h^{-1} P^{\top} & 0 \\
0 & h
\end{array}\right), \quad \omega=\left(\begin{array}{cc}
0 & -P \\
P^{\top} & 0
\end{array}\right), \quad A=\left(\begin{array}{cc}
\left(P L P^{-1}\right)^{\top} & 0 \\
0 & L
\end{array}\right)
$$

where $P=\left(\frac{\partial \mu_{\alpha}}{\partial x_{i}}\right)$ is the Jacobi matrix of the system of functions $\mu_{1}, \ldots, \mu_{\ell}$ (w.r.t. the local coordinates $\left.x_{1}, \ldots, x_{\ell}\right)$.

The following theorem, which describes c-projectively equivalent metrics under the assumption that $A(g, \hat{g})$ has the maximal number of non-constant eigenvalues, shows that in this case the relation between projective equivalence and c-projective equivalence is rather straightforward.

Theorem 1.4. Let $(h, L)$ be a compatible pair on $U$ satisfying $\mathrm{A} 1$ and $\mathrm{A} 2$. Then the above formulas (1.10) and (1.11) (or equivalently (1.12) in matrix form) define a Kähler structure $(g, \omega)$ and a hermitian endomorphism A which are c-compatible, i.e., satisfy (1.4). Conversely, if a 
Kähler structure $(g, \omega)$ and a hermitian endomorphism A are c-compatible and the eigenvalues of A (as a complex endomorphism) satisfy A1 and A2 in a neighbourhood of some point, then locally, in a neighbourhood of this point, $g, \omega$ and $A$ can be written in the form (1.10) and (1.11), where $h=\sum_{i, j} B_{i j}(x) \mathrm{d} x_{i} \mathrm{~d} x_{j}$ and $L=\sum_{i, j} L_{j}^{i} \mathrm{~d} x_{j} \otimes \partial_{x_{i}}$ are compatible.

Example 2. The simplest example of the situation described in Theorem 1.4 is obtained by starting with a 2-dimensional compatible pair $(h, L)$ such that $L$ has two real non-constant eigenvalues $\rho, \sigma$ satisfying A1 and A2. The description of such a pair is due to Dini [16], see also [9]: locally, we find coordinates $x, y$ such that $\rho=\rho(x)$ and $\sigma=\sigma(y)$ and

$$
h=(\rho-\sigma)\left(\mathrm{d} x^{2} \pm \mathrm{d} y^{2}\right), \quad L=\rho \mathrm{d} x \otimes \frac{\partial}{\partial x}+\sigma \mathrm{d} y \otimes \frac{\partial}{\partial y} .
$$

Applying Theorem 1.4 to these formulas, we obtain the formulas for the Kähler structure $(g, \omega)$ and the c-compatible endomorphism $A$. These formulas can be found in $[10,(3.1)$ and $(3.2)]$.

We see that in the situation of Theorem 1.4, the entries of $g, \omega$ and $A$ do not depend on the coordinates $t_{1}, \ldots, t_{\ell}$. This implies that $\frac{\partial}{\partial t_{1}}, \ldots, \frac{\partial}{\partial t_{\ell}}$ are $J$-preserving Killing vector fields, and they are precisely the Killing vector fields $K_{1}, \ldots, K_{\ell}$ which we mentioned in $\S 1.2$. The quotient with respect to the local action of these vector fields is $n$-dimensional with local coordinates $x_{1}, \ldots, x_{\ell}$, and the metric $g$ descends to the metric $g_{Q}=h$ on the quotient. As claimed in the "main observation" of $\S 1.2, g_{Q}$ admits a projectively equivalent metric $\hat{g}_{Q}$ defined by the endomorphism $L$ which also can be treated as the quotient $L=A_{Q}$ of the hermitian endomorphism $A$.

In the next example and theorem, we present the most general local expression which a Kähler structure $(g, \omega)$ together with a solution $A$ to equation (1.4) can take. The construction below combines the previous two cases from Theorem 1.3 and Example 1.

Example 3. We start with two ingredients:

- a compatible pair $h$ and $L$ defined on a domain $U \subset \mathbb{R}^{\ell}$ and satisfying the conditions A1 and A2 as in Example 1, see (1.9);

- a Kähler structure $\left(g_{\mathrm{c}}, \omega_{\mathrm{c}}\right)$ defined on some domain $S$ with a parallel hermitian endomorphism $A_{\mathrm{c}}$ (notice that the eigenvalues of $A_{\mathrm{c}}$ are constant).

In addition, we assume that the eigenvalues of $L$ at each point $p \in U$ are all different from those of $A_{\mathrm{c}}$.

Consider the direct product $V \times U \times S$, where $V \subset \mathbb{R}^{\ell}$ is a certain domain of the same dimension $\ell$ as $U$, and denote local coordinates on $V, U$ and $S$ by $\left(t_{1}, \ldots, t_{\ell}\right),\left(x_{1}, \ldots, x_{\ell}\right)$ and $\left(y_{1}, \ldots, y_{2 k}\right)$ respectively. On this product $V \times U \times S$, we now define a pseudo-Riemannian metric $g$ and a 2 -form $\omega$ :

$$
\begin{aligned}
g & =\sum_{\alpha, \beta=1}^{\ell} H_{\alpha \beta}(x) \theta_{\alpha} \theta_{\beta}+\sum_{i, j=1}^{\ell} B_{i j}(x) \mathrm{d} x_{i} \mathrm{~d} x_{j}+g_{\mathrm{c}}\left(\chi_{L}\left(A_{\mathrm{c}}\right) \cdot, \cdot\right), \\
\omega & =\sum_{\alpha=1}^{\ell} \mathrm{d} \mu_{\alpha} \wedge \theta_{\alpha}+\omega_{\mathrm{c}}\left(\chi_{L}\left(A_{\mathrm{c}}\right) \cdot, \cdot\right),
\end{aligned}
$$

where $\chi_{L}(t)=\operatorname{det}(t \cdot \operatorname{Id}-L)$ is the characteristic polynomial of $L, \theta_{i}=\mathrm{d} t_{i}+\alpha_{i}$ and the 1-forms $\alpha_{i}$ on $S$ are chosen in such a way that $\mathrm{d} \alpha_{i}=(-1)^{i} \omega_{\mathrm{c}}\left(A_{\mathrm{c}}^{\ell-i} \cdot, \cdot\right)$ (which is possible since $\omega_{\mathrm{c}}\left(A_{\mathrm{c}}^{\ell-i} \cdot, \cdot\right)$ is a parallel 2-form on $S$ ). The other ingredients, $H_{\alpha \beta}$ and $\mu_{i}$, are defined as above in Example 1 and in addition we set $\mu_{0}=1$.

Further we define the endomorphism

$$
A=\sum_{\alpha, \beta=1}^{\ell} M_{\alpha}^{\beta}(x) \theta_{\beta} \otimes \frac{\partial}{\partial t_{\alpha}}+\sum_{i, j=1}^{\ell} L_{j}^{i}(x) \mathrm{d} x_{j} \otimes \frac{\partial}{\partial x_{i}}+\sum_{p, q=1}^{2 k}\left(A_{\mathrm{c}}\right)_{p}^{q} \mathrm{~d} y_{p} \otimes\left(\frac{\partial}{\partial y_{q}}-\sum_{i=1}^{\ell} \alpha_{i q} \frac{\partial}{\partial t_{i}}\right)
$$

where $M_{\alpha}^{\beta}=\sum_{i, j} L_{j}^{i} \frac{\partial \mu_{\beta}}{\partial x_{i}} \frac{\partial x_{j}}{\partial \mu_{\alpha}}$ and $\alpha_{i q}$ resp. $\left(A_{\mathrm{c}}\right)_{p}^{q}$ denote the components of $\alpha_{i}$ resp. $A_{\mathrm{c}}$ w.r.t. the coordinates $y_{1}, \ldots, y_{2 k}$, i.e., $\alpha_{i}=\sum_{q} \alpha_{i q} \mathrm{~d} y_{q}$ and $A_{\mathrm{c}}=\sum_{p, q}\left(A_{\mathrm{c}}\right)_{p}^{q} \mathrm{~d} y_{p} \otimes \partial_{y_{q}}$. 
Equivalently, in matrix form (w.r.t. the basis $\theta_{1}, \ldots, \theta_{\ell}, \mathrm{d} x_{1}, \ldots, \mathrm{d} x_{\ell}, \mathrm{d} y_{1}, \ldots, \mathrm{d} y_{2 k}$ ), the above formulas take the form:

$g=\left(\begin{array}{ccc}P h^{-1} P^{\top} & 0 & 0 \\ 0 & h & 0 \\ 0 & 0 & g_{\mathrm{c}} \cdot \chi_{L}\left(A_{\mathrm{c}}\right)\end{array}\right), \quad \omega=\left(\begin{array}{ccc}0 & -P & 0 \\ P^{\top} & 0 & 0 \\ 0 & 0 & \omega_{\mathrm{c}} \cdot \chi_{L}\left(A_{\mathrm{c}}\right)\end{array}\right), \quad A=\left(\begin{array}{ccc}\left(P L P^{-1}\right)^{\top} & 0 & 0 \\ 0 & L & 0 \\ 0 & 0 & A_{\mathrm{c}}\end{array}\right)$

where $P=\left(\frac{\partial \mu_{\alpha}}{\partial x_{i}}\right)$ is the Jacobi matrix of the system of functions $\mu_{1}, \ldots, \mu_{\ell}$ (w.r.t. the local coordinates $\left.x_{1}, \ldots, x_{\ell}\right)$.

Remark 1.2. Each of the 1-forms $\alpha_{i}$ on $S$ is determined by $\left(\omega_{\mathrm{c}}, A_{\mathrm{c}}\right)$ up to adding the differential of a function. However, replacing $\theta_{i}$ by the 1-forms $\tilde{\theta}_{i}=\theta_{i}+\mathrm{d} f_{i}$ in the formulas of Example 3 for functions $f_{i}$ on $S$, it is easy to construct a local transformation $f: M \rightarrow M$ identifying the formulas in Example 3 written down w.r.t. $\theta_{i}$ and $\tilde{\theta}_{i}$ respectively, see also the discussion after Proposition 4.3 below.

We will call a point $p \in M$ regular with respect to a solution $A$ of (1.4), if in a neighbourhood of this point the number of different eigenvalues of $A$ is constant (which implies that the eigenvalues are smooth functions in some neighbourhood of $p$ ), and for each eigenvalue $\rho$ either $\mathrm{d} \rho \neq 0$, or $\rho$ is constant in a neighbourhood of $p$. Clearly, the set $M^{0}$ of regular points is open and dense in $M$. Further (see Lemma 2.2 (4) below) we will see that the number of non-constant eigenvalues of $A$ is the same near every regular point. The following theorem generalises Theorems 1.3 and 1.4:

Theorem 1.5. The metric $g$ and 2-form $\omega$ defined by (1.13) are a Kähler structure and $A$ defined by (1.14) is a hermitian solution of (1.4).

Conversely, let $(M, g, \omega)$ be a Kähler manifold of arbitrary signature and $A$ be a hermitian solution of (1.4). Then in a neighbourhood of a regular point, the Kähler structure $(g, \omega)$ and the endomorphism $A$ can be written in the form (1.13) and (1.14) from Example 3.

Example 4. The simplest example of the situation described in Theorem 1.5 is obtained by starting with a 1-dimensional compatible pair $h=\mathrm{d} x^{2}, L=\rho \mathrm{d} x \otimes \partial_{x}$ for a function $\rho=\rho(x)$ satisfying $\mathrm{d} \rho \neq 0$ and a 2-dimensional Kähler structure $\left(g_{\mathrm{c}}, \omega_{\mathrm{c}}\right)$ with parallel hermitian endomorphism $A_{\mathrm{c}}=c \cdot \mathrm{Id}$ for a constant $c$. Applying Theorem 1.4 to these formulas, we obtain the formulas for the Kähler structure $(g, \omega)$ and the c-compatible endomorphism $A$ given by [10, formulas (3.5) and (3.6)] (up to a slight change of notation).

Theorem 1.5 gives us a description of a c-compatible pair $(g, \omega)$ and $A$ (at a generic point) provided we know a description of compatible pairs $(h, L)$ and also of Kähler structures $\left(g_{\mathrm{c}}, \omega_{\mathrm{c}}\right)$ admitting a parallel hermitian endomorphism $A_{\mathrm{c}}$. As we already mentioned above, the latter have been described in [13]. The local normal forms for compatible pairs $(h, L)$ have been obtained in [9] and this combined with Theorem 1.5 implies the local normal forms for a c-compatible pair $(g, \omega)$ and $A$, see Example 5 and Theorem 1.6 below. We also refer to [10, Theorem 3.1] for the formulas in the 4-dimensional case.

Example 5 (Main example). Let $2 n \geq 4$ and consider an open subset $W$ of $\mathbb{R}^{2 n}$ of the form $W=V \times U \times S_{1} \times \cdots \times S_{N}$ for open subsets $V, U \subseteq \mathbb{R}^{\ell}$ and $S_{\gamma} \subseteq \mathbb{R}^{2 m_{\gamma}}$. Let $t_{1}, \ldots, t_{\ell}$ denote the coordinates on $V$ and let the coordinates on $U$ be separated into $r$ complex coordinates $z_{1}, \ldots, z_{r}$ and $q=\ell-2 r$ real coordinates $x_{r+1}, \ldots, x_{r+q}$.

Suppose the following data is given on these open subsets:

- Kähler structures $\left(g_{\gamma}, J_{\gamma}, \omega_{\gamma}\right)$ on $S_{\gamma}$ for $\gamma=1, \ldots, N$.

- For each $\gamma=1, \ldots, N$, a parallel hermitian endomorphism $A_{\gamma}: T S_{\gamma} \rightarrow T S_{\gamma}$ for $\left(g_{\gamma}, J_{\gamma}\right)$ having a pair of complex conjugate eigenvalues $c_{\gamma}, \bar{c}_{\gamma} \in \mathbb{C} \backslash \mathbb{R}$ for $\gamma=1, \ldots, R$ and a single real eigenvalue $c_{\gamma} \in \mathbb{R}$ for $\gamma=R+1, \ldots, N$ such that the algebraic multiplicity of $c_{\gamma}$ equals $m_{\gamma} / 2$ for $\gamma=1, \ldots, R$ and $m_{\gamma}$ for $\gamma=R+1, \ldots, N$.

- Holomorphic functions $\rho_{j}\left(z_{j}\right)$ of $z_{j}$ for $1 \leq j \leq r$ and smooth functions $\rho_{j}\left(x_{j}\right)$ for $r+1 \leq$ $j \leq r+q$. 
Moreover, we choose 1 -forms $\alpha_{1}, \ldots, \alpha_{\ell}$ on $S=S_{1} \times \cdots \times S_{N}$ which satisfy

$$
\mathrm{d} \alpha_{i}=(-1)^{i} \sum_{\gamma=1}^{N} \omega_{\gamma}\left(A_{\gamma}^{\ell-i} \cdot, \cdot\right) .
$$

We introduce some notation to be used throughout the paper. The function $\Delta_{i}$ for $1 \leq i \leq r+q$ is given by $\Delta_{i}=\prod_{\rho \in E_{\mathrm{nc}} \backslash\left\{\rho_{i}\right\}}\left(\rho_{i}-\rho\right)$, where $E_{\mathrm{nc}}=\left\{\rho_{1}, \bar{\rho}_{1}, \ldots, \rho_{r}, \bar{\rho}_{r}, \rho_{r+1}, \ldots, \rho_{r+q}\right\}$. The 1 -forms $\theta_{1}, \ldots, \theta_{\ell}$ on $W$ are defined by $\theta_{i}=\mathrm{d} t_{i}+\alpha_{i}$. The function $\mu_{i}$ denotes the $i$ th elementary symmetric polynomial in the $\ell$ variables $E_{\mathrm{nc}}, \mu_{i}\left(\hat{\rho_{s}}\right)$ denotes the $i$ th elementary symmetric polynomial in the $\ell-1$ variables $E_{\mathrm{nc}} \backslash\left\{\rho_{s}\right\}$ and the notation "c.c" refers to the conjugate complex of the preceding term.

Suppose that at every point of $W$ the values of the functions $\rho_{1}, \bar{\rho}_{1}, \ldots, \rho_{r+q}$ are mutually different and different from the constants $c_{1}, \bar{c}_{1}, \ldots, c_{N}$ and their differentials are non-zero. Then $(g, \omega, J)$ given by the formulas

$$
\begin{gathered}
g=-\frac{1}{4} \sum_{i=1}^{r}\left(\Delta_{i} \mathrm{~d} z_{i}^{2}+c . c .\right)+\sum_{i=r+1}^{r+q} \varepsilon_{i} \Delta_{i} \mathrm{~d} x_{i}^{2}+\sum_{i=0}^{\ell}(-1)^{i} \mu_{i} \sum_{\gamma=1}^{N} g_{\gamma}\left(A_{\gamma}^{\ell-i} \cdot, \cdot\right) \\
+\sum_{i, j=1}^{\ell}\left[-4 \sum_{s=1}^{r}\left(\frac{\mu_{i-1}\left(\hat{\rho_{s}}\right) \mu_{j-1}\left(\hat{\rho_{s}}\right)}{\Delta_{s}}\left(\frac{\partial \rho_{s}}{\partial z_{s}}\right)^{2}+c . c .\right)+\sum_{s=r+1}^{r+q} \varepsilon_{s} \frac{\mu_{i-1}\left(\hat{\rho}_{s}\right) \mu_{j-1}\left(\hat{\rho}_{s}\right)}{\Delta_{s}}\left(\frac{\partial \rho_{s}}{\partial x_{s}}\right)^{2}\right] \theta_{i} \theta_{j}, \\
\omega=\sum_{i=1}^{\ell} \mathrm{d} \mu_{i} \wedge \theta_{i}+\sum_{i=0}^{\ell}(-1)^{i} \mu_{i} \sum_{\gamma=1}^{N} \omega_{\gamma}\left(A_{\gamma}^{\ell-i} \cdot, \cdot\right), \\
\mathrm{d} z_{i} \circ J=4 \frac{1}{\Delta_{i}} \frac{\partial \rho_{i}}{\partial z_{i}} \sum_{j=1}^{\ell} \mu_{j-1}\left(\hat{\rho}_{i}\right) \theta_{j}, \quad \mathrm{~d} x_{i} \circ J=-\frac{\varepsilon_{i}}{\Delta_{i}} \frac{\partial \rho_{i}}{\partial x_{i}} \sum_{j=1}^{\ell} \mu_{j-1}\left(\hat{\rho}_{i}\right) \theta_{j}, \\
\theta_{i} \circ J=\frac{(-1)^{i}}{4} \sum_{j=1}^{r} \rho_{j}^{\ell-i}\left(\frac{\partial \rho_{j}}{\partial z_{j}}\right)^{-1} \mathrm{~d} z_{j}+c . c+(-1)^{i-1} \sum_{j=r+1}^{r+q} \varepsilon_{j} \rho_{j}^{\ell-i}\left(\frac{\partial \rho_{j}}{\partial x_{j}}\right)^{-1} \mathrm{~d} x_{j}
\end{gathered}
$$

is Kähler, where $\varepsilon_{i}= \pm 1$ depending on the signature of $g$. Moreover, writing $\alpha_{i}=\sum_{q} \alpha_{i q} \mathrm{~d} y_{q}$ and $A_{\gamma}=\sum_{p, q}\left(A_{\gamma}\right)_{p}^{q} \mathrm{~d} y_{p} \otimes \partial_{y_{q}}$ w.r.t. local coordinates $y_{1}, \ldots, y_{2 k}$ on $S=\prod_{\gamma} S_{\gamma}$, we have that the endomorphism $A$ given by

$$
\begin{gathered}
A=\sum_{i, j=1}^{\ell}\left(\mu_{i} \delta_{1 j}-\delta_{i(j-1)}\right) \theta_{i} \otimes \frac{\partial}{\partial t_{j}}+\sum_{s=1}^{r}\left(\rho_{s} \mathrm{~d} z_{s} \otimes \frac{\partial}{\partial z_{s}}+c . c .\right)+\sum_{s=r+1}^{r+q} \rho_{s} \mathrm{~d} x_{s} \otimes \frac{\partial}{\partial x_{s}} \\
+\sum_{\gamma=1}^{N} \sum_{p, q=1}^{2 k}\left(A_{\gamma}\right)_{p}^{q} \mathrm{~d} y_{p} \otimes\left(\frac{\partial}{\partial y_{q}}-\sum_{i=1}^{\ell} \alpha_{i q} \frac{\partial}{\partial t_{i}}\right)
\end{gathered}
$$

is a hermitian solution to (1.4).

Example 5 is an explicit construction of a Kähler metric (1.16) and a solution (1.17) of (1.4). This fact can be verified by a straightforward, though non-trivial computation. Another proof will be given in Sections 4.2 and 4.3. The next theorem shows that in a neighbourhood of a generic point, a Kähler metric $g$ (of any signature) and a solution $A$ of (1.4) are, in a certain coordinate system, as in Example 5.

Theorem 1.6 (Local description of c-projectively equivalent metrics). Suppose $(M, g, J)$ is a Kähler manifold of arbitrary signature and $A$ is a hermitian solution of (1.4). Assume that in a small neighbourhood $W \subseteq M^{0}$ of a regular point, $A$ has

- $\ell=2 r+q$ non-constant eigenvalues on $W$ which separate into $r$ pairs of complex-conjugate eigenvalues $\rho_{1}, \bar{\rho}_{1}, \ldots, \rho_{r}, \bar{\rho}_{r}: W \rightarrow \mathbb{C}$ and $q$ real eigenvalues $\rho_{r+1}, \ldots, \rho_{r+q}: W \rightarrow \mathbb{R}$,

- $N+R$ constant eigenvalues which separate into $R$ pairs of complex conjugate eigenvalues $c_{1}, \bar{c}_{1}, \ldots, c_{R}, \bar{c}_{R}$ and $N-R$ real eigenvalues $c_{R+1}, \ldots, c_{N}$. 
Then the Kähler structure $(g, J, \omega)$ and $A$ are given on $W$ by the formulas (1.16) and (1.17) from Example 5.

Remark 1.3. As stated above, the corresponding local description of a positive definite Kähler structure $(g, J, \omega)$ admitting a hermitian solution $A$ of (1.4) has been obtained in [1] in the language of hamiltonian 2-forms. To obtain a better understanding of the relation between the formulas in [1] and the formulas (1.16) and (1.17) above, note that if $g$ is positive definite, all the eigenvalues of the hermitian endomorphism $A$, both constant and non-constant, are real. Thus, for a positive definite metric $g$, the pairs of conjugate complex non-constant eigenvalues $\rho_{1}, \bar{\rho}_{1}, \ldots, \rho_{r}, \bar{\rho}_{r}$ disappear from the formulas (1.16) and (1.17) and the endomorphisms $A_{\gamma}: T S_{\gamma} \rightarrow T S_{\gamma}$ are just a constant multiple of the identity Id : $T S_{\gamma} \rightarrow T S_{\gamma}$, that is, $A_{\gamma}=c_{\gamma} \cdot$ Id. Moreover, in [1], the non-constant eigenvalues $\rho_{1}, \ldots, \rho_{\ell}$ of $A$ have been used as part of the coordinate system instead of $x_{1}, \ldots, x_{\ell}$ used in (1.16) and (1.17). The change of coordinates $x_{i} \longmapsto \rho_{i}$ for $i=1, \ldots, \ell$ yields

$$
\mathrm{d} x_{i}^{2}=\frac{\mathrm{d} \rho_{i}^{2}}{\Theta_{i}\left(\rho_{i}\right)},
$$

where $\Theta_{i}\left(\rho_{i}\right)=\left(\frac{\partial \rho_{i}}{\partial x_{i}}\left(x_{i}\left(\rho_{i}\right)\right)\right)^{2}$. The functions $F_{i}(t)$ appearing in the local classification of [1] relate to the $\Theta_{i}$ 's by

$$
F_{i}(t)=\Theta_{i}(t) \prod_{\gamma=1}^{N}\left(t-c_{\gamma}\right)^{m_{\gamma}}
$$

The formula for the hamiltonian 2-form $\phi$ (corresponding to $A$ ) is obtained by inserting (1.16) and (1.17) into $\phi=\omega(A \cdot, \cdot)$. Up to changing notations (for instance $\rho_{i} \longmapsto \xi_{i}, \mu_{i} \longmapsto \sigma_{i}$ ), this relates (1.16) and (1.17) in the case when $g$ is positive definite to the description obtained in [1].

Remark 1.4. As mentioned above, Theorem 1.6 yields an "almost" explicit description of a Kähler metric $g$ admitting a c-projectively equivalent metric. What is not described explicitly are the Kähler structures $\left(g_{\gamma}, \omega_{\gamma}\right)$ that admit parallel hermitian endomorphisms $A_{\gamma}$. The formulas for such a triple $\left(g_{\gamma}, \omega_{\gamma}, A_{\gamma}\right)$ in local coordinates can be found in [13].

1.4. Structure of the paper. In $\S 2$, we recall that the existence of a c-projectively equivalent metric $\hat{g}$ for a Kähler metric $g$ implies the existence of a family of independent commuting hamiltonian (w.r.t. the Kähler form $\omega$ ) Killing vector fields $K_{1}, \ldots, K_{\ell}$. These vector fields are also Killing w.r.t. $\hat{g}$.

We can form the quotient of $M$ w.r.t. the local $\mathbb{R}^{\ell}$-action induced by these vector fields and obtain a bundle structure $M \rightarrow Q$ with fibers being the leaves of the foliation generated by the vector fields $K_{i}$. Since $g, \hat{g}$ are invariant w.r.t. the action of the vector fields $K_{i}$ and the orthogonal complements to the fibers w.r.t. $g$ and $\hat{g}$ coincide, they descend to metrics $g_{Q}, \hat{g}_{Q}$ on the quotient. This reduction will be explained in detail in $\S 3$. As we already mentioned, in $\S 1.2$, the crucial observation is that the metrics on the quotient are projectively equivalent. We prove this property in §3.2. More precisely, as explained in Example 1, instead of $\hat{g}_{Q}$ we consider the endomorphism $A_{Q}$ obtained from $g_{Q}$ and $\hat{g}_{Q}$ by (1.6) and check the compatibility condition for the pair $g_{Q}, A_{Q}$.

The local classification of pseudo-Riemannian projectively equivalent metrics, or equivalently, compatible pairs $g_{Q}$ and $A_{Q}$ has been derived in [9]. We apply these results in $\S 4.1$ to obtain the normal forms for $g_{Q}, A_{Q}$ on the quotient. These normal forms are, in fact, simpler than the generic ones for projectively equivalent metrics: the tensor $A=A(g, \hat{g})$ from Theorem 1.6 has nonconstant eigenvalues of (complex) algebraic multiplicity equal to one such that the corresponding tensor $A_{Q}$ on the quotient has no non-trivial Jordan blocks corresponding to the non-constant eigenvalues. This makes the formulas from [9] much easier.

The requirement that $g$ is Kähler and $A$ is hermitian implies that they are completely determined by the reduced objects $g_{Q}$ and $A_{Q}$ on the quotient. In $\S 4.1$ we derive the formulas for $(g, \omega)$ and $A$ which are in essence equivalent to (1.13) and (1.14) (Proposition 4.3). The next step is to show that there are no further restrictions on $g, \omega$ and $A$ so that the formulas from Proposition 4.3 give us a desired local description, see $\S 4.2$. 
Finally in $\S 4.3$, we complete the proof of Theorem 1.6 by deriving the explicit formulas (1.16) and (1.17) from Example 5.

The second part of the article contains the proof of Theorems 1.1 and 1.2. As we already pointed out, there is a close relationship between c-projective and projective equivalence. This makes the proofs of these theorems rather similar. In Section 5 we focus on the proof of the Yano-Obata conjecture (Theorem 1.1) and explain in a series of remarks how this proof can be adapted for the Lichnerowicz conjecture (Theorem 1.2). This is done under one additional algebraic condition: the endomorphism $A$ compatible with the metric $g$ and induced by the projective vector field $v$ has no Jordan blocks with non-constant eigenvalues ${ }^{2}$. The latter case when $A$ admits a "non-constant" Jordan block is treated in Section 6 .

\section{CANonical Killing Vector fields for C-PROJeCtively EqUivalent Metrics}

Let $(M, g, J)$ be a connected Kähler manifold of real dimension $2 n \geq 4$. Since by definition any $A$ which is c-compatible with $(g, J)$ commutes with $J$, we can consider $A$ as an endomorphism of the $n$-dimensional complex vector space $T_{p} M$ (with complex multiplication given by $(a+i b) X=$ $a X+b J X)$. The determinant of $A$ considered as complex endomorphism will be denoted by $\operatorname{det}_{\mathbb{C}} A$. It is a smooth function on $M$ and since $A$ is hermitian, it is real valued. Up to a sign $\operatorname{det}_{\mathbb{C}} A$ equals $\sqrt{\operatorname{det} A}$, though the latter is always positive and smoothness may fail at the points where it vanishes.

Recall that a vector field is called a Killing vector field w.r.t. the metric $g$, if its local flow preserves $g$. Similarly, a vector field is called holomorphic if its local flow preserves the complex structure $J$.

Since the local flow of a holomorphic Killing vector field $K$ preserves the symplectic form $\omega=g(J \cdot, \cdot)$, the vector field $K$ is hamiltonian in a neighbourhood of any point or, more generally, on every simply connected open subset. Recall that a vector field $K$ is called hamiltonian if there exists a function $f$ such that

$$
i_{K} \omega=-\mathrm{d} f \text { or, equivalently, } K=J \operatorname{grad} f .
$$

Such a function $f$ is called a hamiltonian for $K$ and it is only unique up to adding a constant. Conversely, since every hamiltonian vector field preserves $\omega$, it is Killing if and only if it is holomorphic. Recall also that holomorphic vector fields are characterised by the property that their covariant differential is complex-linear (when considered as endomorphism of $T_{p} M$ ) and therefore a hamiltonian vector field $K=J \operatorname{grad} f$ is holomorphic if and only if the hessian $\nabla^{2} f$ is hermitian.

Lemma 2.1. For any $A$ which is c-compatible with $(g, J)$ the function $\operatorname{det}_{\mathbb{C}} A$ is a hamiltonian for a Killing vector field.

We do not pretend that Lemma 2.1 is new: for positive definite metrics it is equivalent to [1, Proposition 3] and this proof can be generalised to all signatures. We give a different and shorter proof, which is based on the same observation as the proof given in [14] but does not require introducing c-projectively invariant objects.

Proof. Since the statement is local, w.l.o.g. we may assume that $\operatorname{det} A \neq 0$, otherwise we can locally replace $A$ by $A+$ const $\cdot$ Id. Then, as explained in $\S 1.3$, the metric $\hat{g}$ given by (1.3) is c-projectively equivalent to $g$. We denote by $\nabla$ and $\hat{\nabla}$ the Levi-Civita connections of $g$ and $\hat{g}$. It is well known (see for example the survey [32]), and follows directly from the definition of c-projective equivalence, that the connections $\nabla$ and $\hat{\nabla}$ are related by the equation

$$
\hat{\nabla}_{X} Y-\nabla_{X} Y=\Phi(X) Y+\Phi(Y) X-\Phi(J X) J Y-\Phi(J Y) J X
$$

where $\Phi$ is an exact 1 -form equal to the differential of the function

$$
\phi=\frac{1}{4(n+1)} \ln \left(\frac{\operatorname{det} \hat{g}}{\operatorname{det} g}\right) .
$$

\footnotetext{
${ }^{2}$ According to the local description given by Theorem 1.6, in the c-projective setting such blocks do not occur.
} 
Combining (1.3) and (2.2), we see that

$$
\exp (-2 \phi)=\left|\operatorname{det}_{\mathbb{C}} A\right|
$$

Now, it follows from straightforward calculations using (2.1) (see e.g. [32]), that the Ricci tensors Ric and $\widehat{\mathrm{Ric}}$ of the metrics $g$ and $\hat{g}$ are related by

$$
\widehat{\mathrm{Ric}}-\mathrm{Ric}=-2(n+1)\left(\nabla \Phi-\Phi^{2}+(\Phi \circ J)^{2}\right) .
$$

Note that $\nabla \Phi$ is a symmetric $(0,2)$-tensor. For a Kähler metric, the Ricci tensor is hermitian w.r.t. the complex structure. Then the above equation implies that $\nabla \Phi-\Phi^{2}+(\Phi \circ J)^{2}$ is hermitian. Hence,

$$
\begin{aligned}
\nabla^{2}\left|\operatorname{det}_{\mathbb{C}} A\right| & =\nabla^{2} \exp (-2 \phi)=2 \exp (-2 \phi)\left(-\nabla \Phi+2 \Phi^{2}\right) \\
& =2 \exp (-2 \phi)\left(-\left(\nabla \Phi-\Phi^{2}+(\Phi \circ J)^{2}\right)+\Phi^{2}+(\Phi \circ J)^{2}\right)
\end{aligned}
$$

is hermitian as well. This implies that $J \operatorname{grad}\left|\operatorname{det}_{\mathbb{C}} A\right|$ is Killing.

For each $A$ which is c-compatible with $(g, J)$, Lemma 2.1 gives us a hamiltonian Killing vector field with the hamiltonian function $\operatorname{det}_{\mathbb{C}} A$. If $A$ is not parallel, this function is non-constant and therefore the Killing vector field is non-trivial.

Since equation (1.4) is linear in $A$ and admits Id : $T M \rightarrow T M$ as a solution, we actually have a whole family $A(t)=t \cdot \operatorname{Id}-A$ of endomorphisms c-compatible with $(g, J)$. For any fixed $t$, the function $\operatorname{det}_{\mathbb{C}} A(t)$ is a hamiltonian for a Killing vector field which we denote by $K(t)$. We will call these vector fields, and also all their linear combinations with constant coefficients, canonical Killing vector fields corresponding to the solution $A$ of (1.4) (or to the c-projectively equivalent metric $\hat{g}$ ), or simply canonical Killing vector fields.

Lemma 2.2. The following statements hold for any endomorphism A which is c-compatible with $(g, J)$ :

(1) Suppose for a smooth function $\rho$ on an open subset $U \subseteq M$ and for any point $p \in U$ the number $\rho(p)$ is an eigenvalue of $A$ at $p$ of algebraic multiplicity $\geq 4$. Then this function $\rho$ is a constant on $U$. Moreover, for any point of the manifold the constant $\rho$ is an eigenvalue of $A$.

(2) The vectors $\operatorname{grad} \rho$ and J $\operatorname{grad} \rho$ are eigenvectors of $A$ with eigenvalue $\rho$ at the points where the eigenvalue $\rho$ is a smooth function.

(3) At a generic point, the number of linearly independent canonical Killing vector fields coincides with the number of non-constant eigenvalues of $A$.

(4) At each regular point the number of eigenvalues $\rho$ with $\mathrm{d} \rho \neq 0$ is the same.

(5) At regular points, the restriction of $g$ to the distribution spanned by the canonical Killing vector fields is non-degenerate.

(6) The canonical Killing vector fields $K(t)$, and also the vector fields $J K(t)$ commute: for any $t_{1}, t_{2} \in \mathbb{R}$ we have

$\left[K\left(t_{1}\right), K\left(t_{2}\right)\right]=\left[K\left(t_{1}\right), J K\left(t_{2}\right)\right]=\left[J K\left(t_{1}\right), J K\left(t_{2}\right)\right]=0 \quad$ and $\quad \omega\left(K\left(t_{1}\right), K\left(t_{2}\right)\right)=0$.

(7) The local flow of every canonical Killing vector field preserves $A$.

(8) For any two canonical Killing vector fields $K\left(t_{1}\right), K\left(t_{2}\right)$ the vector $J \nabla_{K\left(t_{1}\right)} K\left(t_{2}\right)$ at any point is contained in the span of the vector fields $K(t), t \in \mathbb{R}$.

Most statements of the lemma can be found in [14]. The proofs in the present paper are different from those in [14], are shorter and do not require introducing c-projectively invariant objects. For $g$ positive definite, the statements of the lemma have been obtained in the language of hamiltonian 2 -forms in [1]. It is not possible (that is, we did not find an easy way to do it) to directly generalise the proof from [14] to metrics of all signatures.

Proof. Let $\lambda_{1}(x), \ldots, \lambda_{k}(x)$ be the eigenvalues of $A$ at a point $x \in M$. In the proof of the 1 st statement of Lemma 2.2 we will work in a neighbourhood of a generic point, which implies that we may assume w.l.o.g. that the algebraic multiplicities of the eigenvalues are $2 m_{1}, \ldots, 2 m_{k}$, they do not change in this neighbourhood and all $\lambda_{i}$ are smooth, possibly complex-valued functions. 
Now, evidently $f(t)=\operatorname{det}_{\mathbb{C}}(t \cdot \operatorname{Id}-A)=\left(t-\lambda_{1}\right)^{m_{1}} \ldots\left(t-\lambda_{k}\right)^{m_{k}}$. Note that the formula for $f(t)$ makes sense also if $t \in \mathbb{C} \backslash \mathbb{R}$. Indeed, because of linearity of the Killing equation, for a hamiltonian function $f(t)$ with $t \in \mathbb{C} \backslash \mathbb{R}$ the hamiltonian vector field, which is now complex-valued, is still a holomorphic Killing vector field in the sense that its real and imaginary parts are holomorphic Killing vector fields. To see this, note that $f(t)$ is a polynomial in $t$ so all of its coefficients are hamiltonians for holomorphic Killing vector fields. Thus, for complex-valued $t$ the real and imaginary parts of $f(t)$ are still linear combinations of the coefficients.

Consider now the family $\mathrm{d} f(t)$ of differentials of hamiltonians of the canonical Killing vector fields. It is given by

$$
\begin{array}{r}
m_{1}\left(t-\lambda_{1}\right)^{m_{1}-1}\left(t-\lambda_{2}\right)^{m_{2}} \ldots\left(t-\lambda_{k}\right)^{m_{k}} \mathrm{~d} \lambda_{1}+m_{2}\left(t-\lambda_{1}\right)^{m_{1}}\left(t-\lambda_{2}\right)^{m_{2}-1} \ldots\left(t-\lambda_{k}\right)^{m_{k}} \mathrm{~d} \lambda_{2}+ \\
\ldots+m_{k}\left(t-\lambda_{1}\right)^{m_{1}}\left(t-\lambda_{2}\right)^{m_{2}} \ldots\left(t-\lambda_{k}\right)^{m_{k}-1} \mathrm{~d} \lambda_{k} .
\end{array}
$$

Suppose now that a (possibly complex-valued) eigenvalue $\lambda_{i}$ has algebraic multiplicity $2 m_{i} \geq 4$. W.l.o.g. we may think that $i=1$. We take an arbitrary point $p$, set $\tilde{\lambda}=\lambda_{1}(p)$ and consider the hamiltonian $f(t)$ with $t=\tilde{\lambda}$. Since $m_{1} \geq 2$, we see that $\mathrm{d} f(\tilde{\lambda})=0$ at $p$. Then the components of the matrix of the hessian $\nabla^{2} f(\tilde{\lambda})$ at $p$ in any coordinate system $x_{i}$ are simply given by the components $\partial_{i} \partial_{j} f(\tilde{\lambda})$ of the usual hessian at $p$ and, hence,

$$
\nabla^{2} f(\tilde{\lambda})(p)=m_{1}\left(m_{1}-1\right)\left(\tilde{\lambda}-\lambda_{1}\right)^{m_{1}-2}\left(\tilde{\lambda}-\lambda_{2}\right)^{m_{2}} \ldots\left(\tilde{\lambda}-\lambda_{k}\right)^{m_{k}} \mathrm{~d} \lambda_{1}^{2} .
$$

We see that if $\lambda_{1}$ is actually real-valued, the hessian $\nabla^{2} f(\tilde{\lambda})$ at the point $p$ vanishes or has rank 1. But it cannot have rank 1 because it is hermitian. Thus, $\nabla^{2} f(\tilde{\lambda})$ has to vanish.

Suppose now $\lambda_{1}=\alpha+i \beta$, where $\alpha$ and $\beta$ are real-valued functions. Then,

$$
\mathrm{d} \lambda_{1}^{2}=\mathrm{d} \alpha^{2}-\mathrm{d} \beta^{2}+2 i \mathrm{~d} \alpha \mathrm{d} \beta .
$$

If $\mathrm{d} \alpha$ and $\mathrm{d} \beta$ are linearly dependent, $\mathrm{d} \alpha^{2}-\mathrm{d} \beta^{2}$ and $\mathrm{d} \alpha \mathrm{d} \beta$ have rank 1 or 0 . Since rank 1 is impossible they vanish. The case when $\mathrm{d} \alpha$ and $\mathrm{d} \beta$ are linearly independent cannot occur because in this case the bilinear forms $\mathrm{d} \alpha^{2}-\mathrm{d} \beta^{2}$ and $\mathrm{d} \alpha \mathrm{d} \beta$ have signature $(1,1,2 n-2)$, which contradicts that they are hermitian. Finally, $\nabla^{2} f(\tilde{\lambda})=0$ at $p$.

It is well known that the first jet (i.e, the vector field and its first covariant derivative) of a Killing vector field at a point determines the Killing vector field on the whole manifold. As we just proved, the first jet of the Killing vector field corresponding to the hamiltonian $f(\tilde{\lambda})$ vanishes at $p$. Then it vanishes on the whole manifold which implies that the function $f(\tilde{\lambda})$ is a constant. It is clearly zero at the point $p$ so it is identically zero and $\tilde{\lambda}$ is an eigenvalue at every point of the manifold. The 1 st statement of Lemma 2.2 is proved.

Let us now prove the 2nd statement. Denote by $X$ a vector field of eigenvectors corresponding to a non-constant eigenvalue $\rho$ (viewed as a function on the manifold). First observe that for any vector $Y$ we have

$$
(A-\rho \cdot \operatorname{Id}) \nabla_{Y} X=\mathrm{d} \rho(Y) X-g(X, Y) \Lambda-g(X, \Lambda) Y-g(X, J Y) J \Lambda+g(X, J \Lambda) J Y .
$$

To obtain (2.4), take the covariant derivative in the direction of $Y$ of the equation $(A-\rho \cdot \operatorname{Id}) X=0$, substitute (1.4) and rearrange the terms.

Taking $Y$ orthogonal to $X$ and to $J X$, we see that the right hand side of (2.4) is a linear combination of the linearly independent vectors $X, Y$ and $J Y$. Since the left hand side $(A-$ $\rho \cdot \mathrm{Id}) \nabla_{Y} X$ is orthogonal to the kernel of $(A-\rho \cdot \mathrm{Id})$, the coefficient of $X$, which is $\mathrm{d} \rho(Y)$, is zero. Thus, the function $\rho$ is constant in any direction orthogonal to $X$ and to $J X$. By the 1 st statement of Lemma 2.2, the algebraic multiplicity of $\rho$ is 2 and it follows that $\operatorname{grad} \rho \operatorname{and} J \operatorname{grad} \rho$ are eigenvectors of $A$ corresponding to the eigenvalue $\rho$.

To prove the 3rd statement, consider the non-constant eigenvalues of $A$ and denote them by $\rho_{1}, \ldots, \rho_{\ell}$. We will work near a generic point so we may assume that $\rho_{1}, \ldots, \rho_{\ell}$ are smooth functions with non-zero differentials. Observe that for any $t$ the function $\left(t-\lambda_{i}\right)^{m_{i}}$ is a constant if the eigenvalue $\lambda_{i}$ is a constant and, in view of the proved 1st statement, if $m_{i} \geq 2$. Then each $f(t)$ is proportional with a constant coefficient to $\left(t-\rho_{1}\right) \ldots\left(t-\rho_{\ell}\right)$. The function $\tilde{f}(t)=\left(t-\rho_{1}\right) \ldots\left(t-\rho_{\ell}\right)$ 
is a polynomial of degree $\ell$ with leading coefficient equal to 1 and has at most $\ell$ non-constant coefficients. Thus, the number of linearly independent canonical Killing vector fields is at most $\ell$.

Since the gradients grad $\rho_{i}$ belong to different eigenspaces, they are linearly independent and in view of $(2.3)$, the differentials of $\tilde{f}\left(t_{1}\right)$ and $\tilde{f}\left(t_{2}\right)$ are linearly independent for $t_{1} \neq t_{2}$ so the number of linearly independent canonical Killing vector fields is precisely $\ell$.

To prove the 4th statement, recall that a Killing vector field which vanishes on an open set vanishes everywhere. Then, by the 3rd statement of the lemma, the number of non-constant eigenvalues is the same on every open subset. Then the number of constant eigenvalues is also the same on every open subset and the claim follows.

In order to proof the 5 th statement, observe that the distribution spanned by the canonical Killing vector fields, at regular points, coincides with the distribution spanned by the hamiltonian vector fields generated by the non-constant eigenvalues. By the 2nd statement, such hamiltonian vector fields have non-zero length at regular points and are mutually orthogonal, and the claim follows.

Let us prove the 6th statement. By the 2nd statement, we have

$$
\omega\left(K\left(t_{1}\right), K\left(t_{2}\right)\right)=g\left(J K\left(t_{1}\right), K\left(t_{2}\right)\right)=0
$$

for any real numbers $t_{1}, t_{2}$. By definition of a Poisson bracket, this equation is equivalent to say that the hamiltonian functions $f\left(t_{1}\right), f\left(t_{2}\right)$ corresponding to $K\left(t_{1}\right), K\left(t_{2}\right)$ Poisson commute, $\left\{f\left(t_{1}\right), f\left(t_{2}\right)\right\}=0$. On the other hand, recall that $\left[K\left(t_{1}\right), K\left(t_{2}\right)\right]$ is the hamiltonian vector field corresponding to the hamiltonian $\left\{f\left(t_{1}\right), f\left(t_{2}\right)\right\}$. We obtain $\left[K\left(t_{1}\right), K\left(t_{2}\right)\right]=0$. The remaining equations follow from the fact that the vector field $K(t)$ is holomorphic and therefore also $J K(t)$ is holomorphic.

To prove the 7th statement, assume w. l. o. g. that $A$ is non-degenerate. Then we can consider the metric $\hat{g}$ from (1.3) c-projectively equivalent to $g$. It is sufficient to show that the canonical Killing vector fields for $g$ are also canonical Killing vector fields for $\hat{g}$. W.l.o.g. we may work in a neighbourhood of a regular point. Let $\rho_{1}, \ldots, \rho_{\ell}$ denote the non-constant eigenvalues of $A$. If we swap the metrics $g$ and $\hat{g}$ in the definition (1.2), the tensor constructed by the pair of metrics $\hat{g}$, $g$ is clearly the inverse of the initial $A$, therefore its non-constant eigenvalues are $\frac{1}{\rho_{1}}, \ldots, \frac{1}{\rho_{\ell}}$.

We will show that the canonical Killing vector field $K(t)$ for $g$, whose hamiltonian is $\operatorname{det}_{\mathbb{C}}(t$. Id $-A$ ), is proportional with a non-zero constant coefficient to the canonical Killing vector field $\hat{K}\left(\frac{1}{t}\right)$ for $\hat{g}$, whose hamiltonian is $\operatorname{det}_{\mathbb{C}}\left(\frac{1}{t} \cdot \operatorname{Id}-A^{-1}\right)$.

Since the multiplicity of the non-constant eigenvalues of $A$ is two, up to multiplication by a constant, for any $t$, the differential of $\operatorname{det}_{\mathbb{C}}(t \cdot \operatorname{Id}-A)$ coincides with the differential of $(t-$ $\left.\rho_{1}\right) \ldots\left(t-\rho_{\ell}\right)$ which is

$$
\left(t-\rho_{2}\right) \ldots\left(t-\rho_{\ell}\right) \mathrm{d} \rho_{1}+\left(t-\rho_{1}\right)\left(t-\rho_{3}\right) \ldots\left(t-\rho_{\ell}\right) \mathrm{d} \rho_{2}+\cdots+\left(t-\rho_{1}\right) \ldots\left(t-\rho_{\ell-1}\right) \mathrm{d} \rho_{\ell} .
$$

Similarly, for any $t \neq 0$, the differential of $\operatorname{det}_{\mathbb{C}}\left(\frac{1}{t} \cdot \operatorname{Id}-A^{-1}\right)$ is proportional with a constant coefficient to the differential of $\left(\frac{1}{t}-\frac{1}{\rho_{1}}\right) \ldots\left(\frac{1}{t}-\frac{1}{\rho_{\ell}}\right)$ which is, up to multiplication by a non-zero constant, given by

$\frac{1}{\operatorname{det}_{\mathbb{C}}(A)}\left(\left(\rho_{2}-t\right) \ldots\left(\rho_{\ell}-t\right) \frac{1}{\rho_{1}} \mathrm{~d} \rho_{1}+\left(\rho_{1}-t\right)\left(\rho_{3}-t\right) \ldots\left(\rho_{\ell}-t\right) \frac{1}{\rho_{2}} \mathrm{~d} \rho_{2}+\cdots+\left(\rho_{1}-t\right) \ldots\left(\rho_{\ell-1}-t\right) \frac{1}{\rho_{\ell}} \mathrm{d} \rho_{\ell}\right)$.

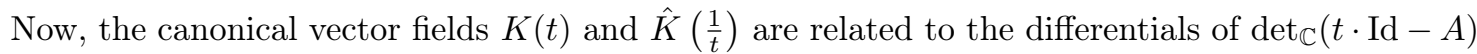
and $\operatorname{det}_{\mathbb{C}}\left(\frac{1}{t} \cdot \mathrm{Id}-A^{-1}\right)$ by

$$
K(t)=\operatorname{Jgrad}_{g} \operatorname{det}_{\mathbb{C}}(t \cdot \operatorname{Id}-A) \text { and } \hat{K}\left(\frac{1}{t}\right)=\operatorname{Jgrad}_{\hat{g}} \operatorname{det}_{\mathbb{C}}\left(\frac{1}{t} \cdot \operatorname{Id}-A^{-1}\right) .
$$

Combining this with (1.3) and the 2 nd statement, we conclude that $K(t)$ is proportional to $\hat{K}\left(\frac{1}{t}\right)$ with a constant factor.

Let us now prove the 8th statement. It is sufficient to prove it on the dense and open subset $M^{0}$ of regular points. As usual, by $\rho_{1}, \ldots, \rho_{\ell}$ we denote the non-constant eigenvalues of $A$. From the definition, it follows that the integrable distribution $\mathcal{V}$ spanned by the canonical Killing vector fields $K(t), t \in \mathbb{R}$, coincides with the distribution spanned by the vector fields $J \operatorname{grad} \rho_{i}, i=1, \ldots, \ell$. Consider the distribution $\mathcal{F}=\mathcal{V} \oplus J \mathcal{V}$. It is spanned by the family of vector fields $K(t), J K(t), t \in$ 
$\mathbb{R}$, is integrable by the 6 th statement and coincides with the span of $\operatorname{grad} \rho_{i}, J \operatorname{grad} \rho_{i}, i=1, \ldots, \ell$. From formula (2.4) combined with the 2nd statement, it follows immediately that the distribution $\mathcal{F}$ is totally geodesic. By the 5th statement, the restriction $\left.g\right|_{\mathcal{L}}$ of $g$ to an integral leaf $\mathcal{L} \subseteq M^{0}$ of $\mathcal{F}$ is nondegenerate. Then it follows that the integral leafs $\tilde{\mathcal{L}} \subseteq \mathcal{L}$ of the integrable subdistribution $J \mathcal{V} \subset \mathcal{F}$ are totally geodesic since they are orthogonal in $\left(\mathcal{L},\left.g\right|_{\mathcal{L}}\right)$ to a distribution spanned by Killing vector fields. This implies that $\nabla_{J K\left(t_{1}\right)} J K\left(t_{2}\right)$ is tangent to $J \mathcal{V}$, or equivalently (since $J$ is parallel and $K(t)$ is holomorphic), that $J \nabla_{K\left(t_{1}\right)} K\left(t_{1}\right)$ is tangent to $\mathcal{V}$ as we claimed. This completes the proof of Lemma 2.2 .

Let $\mu_{i}$ denote the $i$ th elementary symmetric polynomial in $\rho_{1}, \ldots, \rho_{\ell}$, i.e., in the non-constant eigenvalues of $A$ c-compatible with $(g, J)$. Note that although the $\rho_{i}$ may fail to be smooth at certain points, the $\mu_{i}$ are globally defined smooth functions on $M$ : clearly we have $\operatorname{det}_{\mathbb{C}}(t \cdot \operatorname{Id}-A)=$ $P(t) \sum_{i=0}^{\ell}(-1)^{i} \mu_{i} t^{\ell-i}$, where $P(t)$ is a polynomial of degree $n-\ell$ with constant coefficients and we put $\mu_{0}=1$. In what follows we will mainly work with a special set of canonical Killing vector fields $K_{1}, \ldots, K_{\ell}$ corresponding to $A$, where $K_{i}$ is defined to be the hamiltonian vector field with $\mu_{i}$ as a hamiltonian function, i.e.,

$$
K_{i}=J \operatorname{grad} \mu_{i} .
$$

These Killing vector fields have been considered in [1]. By Lemma 2.2, the span of these vector fields at each point coincides with the span of the vector fields $K(t), t \in \mathbb{R}$, and therefore, they share all the properties that have been proven for the vector fields $K(t)$ in Lemma 2.2. For instance, $\omega\left(K_{i}, K_{j}\right)=0$, hence, $\left[K_{i}, K_{j}\right]=\left[J K_{i}, K_{j}\right]=\left[J K_{i}, J K_{j}\right]=0$ and $K_{1} \wedge \cdots \wedge K_{\ell} \neq 0$ at each point of $M^{0}$.

\section{Reduction to the REAL PROJECtive SETting}

We recall the description of a Kähler manifold with a local isometric hamiltonian $\mathbb{R}^{\ell}$-action in §3.1. In the setting of c-projectively equivalent Kähler metrics, this action is given by the commuting Killing vector fields $K_{1}, \ldots, K_{\ell}$ from (2.5) induced by a hermitian solution $A$ of (1.4). As stated in $\S 1.2$, the quotient of the Kähler manifold $(M, g, J)$ w.r.t. to this action yields a manifold $\left(Q, g_{Q}\right)$ and $g_{Q}$ admits a projectively equivalent metric. This will be described in detail in $\S 3.2$.

3.1. The Kähler quotient w.r.t. a local isometric hamiltonian $\mathbb{R}^{\ell}$-action. Recall from $[1, \S 3.1]$ that a local isometric hamiltonian $\mathbb{R}^{\ell}$-action on a Kähler manifold $(M, g, J)$ is given by holomorphic Killing vector fields $K_{1}, \ldots, K_{\ell}$ satisfying

$$
\omega\left(K_{i}, K_{j}\right)=0
$$

and $K_{1} \wedge \cdots \wedge K_{\ell} \neq 0$ on a dense and open subset $M^{0} \subset M$ called the set of regular points.

Note that in [1], the name " $\ell$-torus action" was used instead of " $\mathbb{R}^{\ell}$-action". The point is that the metrics in $[1,2]$ are positive definite so that under the additional assumption of compactness, the isometric $\mathbb{R}^{\ell}$-action described above generates a commutative subgroup of the compact group of isometries, its closure being a torus.

The conditions above imply that the vector fields $K_{1}, \ldots, K_{\ell}$ commute with each other and that they are locally hamiltonian, i.e. we have $K_{i}=J \operatorname{grad} \mu_{i}$ for certain local functions $\mu_{i}, i=1, \ldots, \ell$.

By Lemma 2.2, the canonical Killing vector fields (2.5) coming from a solution $A$ of (1.4) generate a local isometric hamiltonian $\mathbb{R}^{\ell}$-action, where $\ell$ is the number of non-constant eigenvalues of $A$ at a regular point. The notion of regular points as introduced above coincides with the notion of regular points introduced in $\S 1.3$. However, for the time being, we will forget about c-projective geometry and will first restrict to the general setting of a local isometric hamiltonian $\mathbb{R}^{\ell}$-action.

Since we are dealing with metrics of arbitrary signature, we have to take care of the nondegeneracy of orbits of an $\mathbb{R}^{\ell}$-action. A local isometric hamiltonian $\mathbb{R}^{\ell}$-action given by Killing vector fields $K_{1}, \ldots, K_{\ell}$ is called non-degenerate if the restriction of the metric $g$ to the (regular) distribution

$$
\mathcal{V}=\operatorname{span}\left\{K_{1}, \ldots, K_{\ell}\right\}
$$


on the regular set $M^{0}$ is non-degenerate.

As shown in Lemma $2.2(5)$, the $\mathbb{R}^{\ell}$-action coming from the canonical Killing vector fields corresponding to a solution $A$ of (1.4) is non-degenerate in the above sense.

Given a local isometric non-degenerate hamiltonian $\mathbb{R}^{\ell}$-action, we will now reduce the setting by considering the quotient of $M$ w.r.t. the action of the Killing vector fields. The procedure of this reduction and the local description of Kähler metrics admitting a local isometric hamiltonian $\mathbb{R}^{\ell}$-action can be found in $[1, \S 3.1]$ and [36]. For the sake of completeness, we will recall these results. The only difference in our case is that the metric $g$ is allowed to have arbitrary signature but assuming non-degeneracy, there is actually no difference to the procedure described in [1].

Consider a non-degenerate local isometric hamiltonian $\mathbb{R}^{\ell}$-action on $(M, g, J)$ by holomorphic Killing vector fields $K_{1}, \ldots, K_{\ell}$. Let us restrict our attention to the regular set $M^{0}$ and let $G$ denote the commutative (pseudo-)group generated by the local flows of $K_{1}, \ldots, K_{\ell}$. Consider the (local) quotient $Q=M / G$ of $M$ w.r.t. the $G$-action and the (local) fiber bundle

$$
\pi: M \rightarrow Q=M / G \text {. }
$$

The vertical distribution of this bundle coincides with the distribution $\mathcal{V}$ and we define a $(G$ invariant) horizontal distribution $\mathcal{Q}=\mathcal{V}^{\perp}$. Let $\theta=\left(\theta_{1}, \ldots, \theta_{\ell}\right): T M \rightarrow \mathbb{R}^{\ell}$ be the corresponding connection 1-form on $M$, where the components $\theta_{i}$ have been chosen to be dual to the Killing vector fields $K_{i}$, that is, the 1 -forms $\theta_{i}$ are defined by

$$
\theta_{i}\left(K_{j}\right)=\delta_{i j} \text { and } \theta_{i}(\mathcal{Q})=0 .
$$

As above, the local generators for the vector fields $K_{i}$ will be denoted by $\mu_{i}$ (so that $K_{i}=$ $\left.J \operatorname{grad} \mu_{i}\right)$ and we can gather these functions into a (locally defined) moment map $\mu=\left(\mu_{1}, \ldots, \mu_{\ell}\right)$ : $M \rightarrow\left(\mathbb{R}^{\ell}\right)^{*}$ for the hamiltonian action of $G$. Lemma $2.2(6)$ implies that the moment map $\mu$ is $G$-invariant, thus it descends to a mapping $\mu: Q \rightarrow\left(\mathbb{R}^{\ell}\right)^{*}$ on the quotient. The level sets $S_{\mu}$ in $Q$ of this mapping are the Kähler quotients of $(M, g, J)$ w.r.t. the isometric hamiltonian action of $G$. We refer the reader to $[20, \S 3]$ for some background on symplectic reduction and Kähler quotients.

On the other hand, we can also take the (local) quotient of $M$ w.r.t. the action of the commutative (pseudo-)group $G^{\mathbb{C}}$ generated by the local flows of the commuting vector fields $K_{1}, \ldots, K_{\ell}, J K_{1}, \ldots, J K_{\ell}$. The result is a manifold $S=M / G^{\mathbb{C}}$ and since the tangent spaces of the fibers of the bundle $M \rightarrow S$ are $J$-invariant and the action of $G^{\mathbb{C}}$ is by holomorphic transformations, $S$ inherits a canonical complex structure $J_{S}$. As a complex manifold, $S$ can canonically be identified with the Kähler quotients $S_{\mu}$. In view of this, $S$ carries a family of Kähler structures $\left(g_{\mu}, \omega_{\mu}\right)$ which are compatible with the complex structure $J_{S}$. The quotient $Q$ may locally be written in the form $Q=S \times U$, where the open subset $U \subseteq\left(\mathbb{R}^{\ell}\right)^{*}$ parametrises the level sets of $\mu$. In this picture, the subset $U$ can be viewed as the parameter space for the family of compatible Kähler structures $\left(g_{\mu}, \omega_{\mu}\right)$ on $S$.

Since the forms $\theta_{i} \circ J$ and $\mathrm{d} \mu_{i}$ span the same subspace of $T^{*} M$, we can define a point-wise non-degenerate matrix of functions $G_{i j}$ and its inverse with components $H_{i j}$ by

$$
\theta_{i} \circ J=\sum_{j=1}^{\ell} G_{i j} \mathrm{~d} \mu_{j} \text { and } \mathrm{d} \mu_{i} \circ J=-\sum_{j=1}^{\ell} H_{i j} \theta_{j} .
$$

Note that it follows from (3.1) that

$$
H_{i j}=g\left(K_{i}, K_{j}\right)
$$

in particular, $H_{i j}$ and $G_{i j}$ are symmetric in $i, j$.

The Kähler structure can now be written in the form

$$
\begin{gathered}
g=g_{\mu}+\sum_{i, j=1}^{\ell} H_{i j} \theta_{i} \theta_{j}+\sum_{i, j=1}^{\ell} G_{i j} \mathrm{~d} \mu_{i} \mathrm{~d} \mu_{j}, \\
\omega=\omega_{\mu}+\sum_{i=1}^{\ell} \mathrm{d} \mu_{i} \wedge \theta_{i} .
\end{gathered}
$$

In our case, the $\mathbb{R}^{\ell}$-action induced by a solution $A$ of (1.4) satisfies one additional property called rigidity that essentially simplifies the above local formulas for $g$ and $\omega$. 
Recall from $[1, \S 3.2]$ that a local hamiltonian $\mathbb{R}^{\ell}$-action on a Kähler manifold $(M, g, J)$ given by holomorphic Killing vector fields $K_{1}, \ldots, K_{\ell}$ is called rigid if the leaves of the distribution

$$
\mathcal{F}=\mathcal{V} \oplus J \mathcal{V}
$$

are totally geodesic (where $\mathcal{V}$ is the vertical distribution of $M \rightarrow Q$ ).

There are a number of equivalent conditions to this rigidity property, see [1, Proposition 8]. We recall this result for convenience of the reader. Note that, although it has been proven in [1] for positive signature, the proof still works in arbitrary signature assuming non-degeneracy of the $\mathbb{R}^{\ell}$-action.

Proposition 3.1. [1] Consider a local isometric hamiltonian $\mathbb{R}^{\ell}$-action given by holomorphic Killing vector fields $K_{1}, \ldots, K_{\ell}$. The following assumptions are equivalent:

(1) The action is rigid.

(2) The functions $H_{i j}=g\left(K_{i}, K_{j}\right)$ are constant on the level surfaces of the moment map $\mu: M \rightarrow\left(\mathbb{R}^{\ell}\right)^{*}$.

(3) $\nabla_{K_{i}} K_{j} \in \mathcal{F}$ for all $i, j=1, \ldots, \ell$.

(4) The Kähler quotient forms $\omega_{\mu}$ depend affinely on the components $\mu_{i}$ of the moment map $\mu: Q \rightarrow\left(\mathbb{R}^{\ell}\right)^{*}$ and their linear part pulls back to the curvature of $\left(\theta_{1}, \ldots, \theta_{\ell}\right)$.

Remark 3.1. Condition (3) of the proposition can be replaced by " $\nabla_{K_{i}} K_{j} \in J \mathcal{V}$ for all $i, j=$ $1, \ldots, \ell$ ". Indeed, if this holds, $\mathcal{F}$ is obviously totally geodesic, hence, the action is rigid. The converse direction follows from the same line of arguments that has been used in the proof of Lemma $2.2(8)$. We see that " $J \mathcal{V}$ is totally geodesic" is another condition equivalent to rigidity of the action.

Proposition 3.1 gives rise to some simplifications in (3.2) and we come to the following local description:

Proposition 3.2. Let $(M, g, J, \omega)$ be a Kähler $2 n$-manifold together with a rigid non-degenerate (local) isometric hamiltonian $\mathbb{R}^{\ell}$-action generated by Hamiltonian Killing vector fields $K_{i}=J \operatorname{grad} \mu_{i}$, $i=1, \ldots, \ell$. Then locally $M$ can be presented as direct product

$$
V\left(t_{1}, \ldots, t_{\ell}\right) \times U\left(\mu_{1}, \ldots, \mu_{\ell}\right) \times S\left(y_{1}, \ldots, y_{2 k}\right),
$$

and $g, \omega$ and $J$ take the following form:

$$
\begin{gathered}
g=\sum_{i, j=1}^{\ell} H_{i j}(\mu) \theta_{i} \theta_{j}+\sum_{i, j=1}^{\ell} G_{i j}(\mu) \mathrm{d} \mu_{i} \mathrm{~d} \mu_{j}+\sum_{i=1}^{\ell} \mu_{i} g_{i}+g_{0}, \sum_{j=1}^{\ell} H_{i j} G_{j k}=\delta_{i k} \\
\omega=\sum_{i=1}^{\ell} d \mu_{i} \wedge \theta_{i}+\sum_{i=1}^{\ell} \mu_{i} \omega_{i}+\omega_{0}
\end{gathered}
$$

and

$$
\theta_{i} \circ J=\sum_{j=1}^{\ell} G_{i j} \mathrm{~d} \mu_{j}, \quad \mathrm{~d} \mu_{i} \circ J=-\sum_{j=1}^{\ell} H_{i j} \theta_{j}, \quad \mathrm{~d} y_{i} \circ J=\mathrm{d} y_{i} \circ J_{S} .
$$

where the ingredients in these formulas are as follows:

(1) $\theta_{i}=\mathrm{d} t_{i}+\alpha_{i}$ with $\mathrm{d} \alpha_{i}=\omega_{i}$;

(2) $\left(g_{\mu}=\sum_{i} \mu_{i} g_{i}+g_{0}, \omega_{\mu}=\sum_{i} \mu_{i} \omega_{i}+\omega_{0}, J_{S}\right)$, is a Kähler structure on $S$ for any $\mu \in U$ (compatible with the same complex structure $J_{S}$ independent of $\mu$ );

(3) $\partial_{\mu_{i}} G_{j k}=\partial_{\mu_{j}} G_{i k}$.

Conversely, if on $M=V \times U \times S$ we consider $g, \omega, J$ as above, then $(g, \omega)$ is a Kähler structure on $M$ and the generators $\mu_{1}, \ldots, \mu_{\ell}$ define a rigid non-degenerate (local) isometric hamiltonian $\mathbb{R}^{\ell}$-action. In particular, the vector fields $K_{i}=J \operatorname{grad} \mu_{i}$ are holomorphic Killing vector fields. 
3.2. Reduction from the c-projective to the projective setting. We continue to use the notation introduced in the preceding section but assume that the nondegenerate local isometric hamiltonian $\mathbb{R}^{\ell}$-action is given by the Killing vector fields $K_{1}, \ldots, K_{\ell}$ from $(2.5)$ that come from a certain solution $A$ of (1.4). Let $g_{Q}$ denote the metric on the quotient $Q=M / G$ obtained from $g$. In the notation of Proposition 3.2, $Q$ can locally be identified with $U \times S$ and $g_{Q}$ can be obtained from (3.3) by removing the first term, i.e.

$$
g_{Q}=\sum_{i, j=1}^{\ell} G_{i j}(\mu) \mathrm{d} \mu_{i} \mathrm{~d} \mu_{j}+\sum_{i=1}^{\ell} \mu_{i} g_{i}+g_{0} .
$$

Recall that the vertical distribution $\mathcal{V}=\operatorname{span}\left\{K_{1}, \ldots, K_{\ell}\right\}$ coincides with the span of the vector fields $J \operatorname{grad} \rho_{1}, \ldots, J \operatorname{grad} \rho_{\ell}$, where $\rho_{1}, \ldots, \rho_{\ell}$ are the non-constant eigenvalues of $A$. Since by Lemma 2.2 , the vector fields $J \operatorname{grad} \rho_{i}$ take values in the eigenspaces of $A$, the distribution $\mathcal{V}$ is $A$-invariant and consequently, $A$ preserves also $\mathcal{Q}=\mathcal{V}^{\perp}$. On the other hand, according to Lemma $2.2(7), A$ is preserved by the Killing vector fields $K_{i}$ and it follows that $A$ descends to a $g_{Q}$-selfadjoint endomorphism $A_{Q}: T Q \rightarrow T Q$.

Recall the O'Neill formula [34] for a Riemannian submersion relating the Levi-Civita connection $\nabla^{Q}$ of the quotient metric $g_{Q}$ to the Levi-Civita connection $\nabla$ of $g$ by

$$
\nabla_{X}^{Q} Y=\operatorname{pr}_{\mathcal{Q}}\left(\nabla_{X} Y\right)
$$

where $\operatorname{pr}_{\mathcal{Q}}: T M \rightarrow \mathcal{Q}$ is the projection onto the horizontal distribution $\mathcal{Q}$ and we adopted the convention to denote vector fields on $Q$ and their horizontal lifts to $\mathcal{Q}$ by the same symbol. Note that the vector field $\Lambda$ in (1.4) is tangent to the horizontal distribution $\mathcal{Q}$ and it is invariant w.r.t. the action of the $K_{i}$ 's. Thus, $\Lambda$ is (the horizontal lift of) a vector field on $Q$.

Lemma 3.3. The endomorphism $A_{Q}: T Q \rightarrow T Q$ obtained from $A$ by reduction satisfies the equation

$$
\nabla_{X}^{Q} A_{Q}=X^{b} \otimes \Lambda+\Lambda^{b} \otimes X
$$

for all $X \in T Q$, where $X^{b}=g_{Q}(X, \cdot)$. In other words, $g_{Q}$ and $A_{Q}$ are compatible in the projective sense.

Proof. From the O'Neill formula (3.7), the definition of $A_{Q}$ and commutativity of $A$ with $\mathrm{pr}_{\mathcal{Q}}$, it follows that

$$
\left(\nabla_{X}^{Q} A_{Q}\right) Y=\nabla_{X}^{Q}\left(A_{Q} Y\right)-A_{Q}\left(\nabla_{X}^{Q} Y\right)=\operatorname{pr}_{\mathcal{Q}}\left(\left(\nabla_{X} A\right) Y\right)
$$

Inserting (1.4) into this equation and using the fact that $J \Lambda$ is tangent to $\mathcal{V}=\mathcal{Q}^{\perp}$, we obtain

$$
\left(\nabla_{X}^{Q} A_{Q}\right) Y=g(X, Y) \Lambda+g(\Lambda, Y) X=g_{Q}(X, Y) \Lambda+g_{Q}(\Lambda, Y) X
$$

as we claimed.

The local description of compatible pairs $\left(g_{Q}, A_{Q}\right)$ has been recently obtained in [9] (see also [8]). These results, after some adaptation, will lead us to the local description of pairs $(g, A)$.

\section{LoCAL DESCRIPTION OF C-PROJECTIVELY EQUIVALENT METRICS}

In this section we prove Theorems 1.5 and 1.6 .

4.1. Local description of the quotients of c-projectively equivalent metrics and lifting. We have shown above that by taking the quotient of $M$ w.r.t. the action of the Killing vector fields $K_{1}, \ldots, K_{\ell}$, the local description of a Kähler manifold $(M, g, J)$ of arbitrary signature admitting a hermitian solution $A$ of (1.4) is reduced to the classification of pseudo-Riemannian manifolds $\left(Q, g_{Q}\right)$ admitting a $g_{Q}$-selfadjoint solution $A_{Q}$ to (3.8). In other words, a description of c-compatible pairs $g, A$ is reduced to a similar problem for compatible pairs $g_{Q}, A_{Q}$ on the quotient $Q=M / G$ which has been solved in [9] and we apply this result to our situation.

Before deriving the local description for the pair $\left(g_{Q}, A_{Q}\right)$ in our specific situation, we briefly recall the "splitting and gluing constructions" from [8] appropriately reformulated for our purposes, we refer to $[9, \S 1.2]$ for a more detailed summary. 
Let $\left(Q, g_{Q}\right)$ be a pseudo-Riemannian manifold and $A_{Q}: T Q \rightarrow T Q$ be a $g_{Q}$-selfadjoint endomorphism compatible with $g_{Q}$ in the projective sense, i.e., satisfying (3.8).

In a neighbourhood of a generic point, the eigenvalues of $A_{Q}$ are smooth (possibly complex valued functions). Some of them, say $c_{1}, \ldots, c_{n}$, are constant. Then the characteristic polynomial $\chi(t)=\operatorname{det}\left(t \cdot \operatorname{Id}-A_{Q}\right)$ of $A_{Q}$ can be written as

$$
\chi(t)=\chi_{\mathrm{nc}}(t) \cdot \chi_{\mathrm{c}}(t)
$$

where the roots of $\chi_{\mathrm{c}}$ are the constant eigenvalues of $A_{Q}$ (with multiplicities), whereas the roots of $\chi_{\mathrm{nc}}$ are the non-constant eigenvalues. Assume that these polynomials $\chi_{\mathrm{nc}}(t)$ and $\chi_{\mathrm{c}}(t)$ are relatively prime, i.e., the non-constant eigenvalues cannot take the values $c_{1}, \ldots, c_{n}$. In other words, we divide the spectrum of $A_{Q}(p), p \in Q$ into the "constant" and "non-constant" parts, and assume that these parts are disjoint for any $p \in Q$.

Proposition 4.1. [8] Locally $Q$ can be presented as $U\left(x_{1}, \ldots, x_{\ell}\right) \times S\left(y_{1}, \ldots, y_{s}\right)$ so that the endomorphism $A_{Q}$ and the metric $g_{Q}$ take the following block-diagonal form

$$
A_{Q}(x, y)=\left(\begin{array}{cc}
L(x) & 0 \\
0 & A_{\mathrm{c}}(y)
\end{array}\right) \quad \text { and } \quad g_{Q}(x, y)=\left(\begin{array}{cc}
h(x) & 0 \\
0 & g_{\mathrm{c}}(y) \cdot \chi_{\mathrm{nc}}\left(A_{\mathrm{c}}(y)\right)
\end{array}\right),
$$

where $L$ and $h$ are compatible (that is, satisfy (1.7)) on $U$, and $A_{\mathrm{c}}$ is parallel on $S$ w.r.t. $g_{\mathrm{c}}$.

Conversely, $A_{Q}(x, y)$ and $g_{Q}(x, y)$ defined by (4.1) are compatible in the projective sense, i.e., satisfy (3.8) on $U \times S$, whenever $h$ and $L$ are compatible, $A_{\mathrm{c}}$ is parallel w.r.t. $g_{\mathrm{c}}$ and the spectra of $L$ and $A_{\mathrm{c}}$ are disjoint.

Notice that the formula for the metric $g_{Q}$ can be equivalently rewritten as follows

$$
g_{Q}=\sum_{i, j=1}^{\ell} B_{i j}(x) \mathrm{d} x_{i} \mathrm{~d} x_{j}+\sum_{i=1}^{\ell} \mu_{i}(x) g_{i}+g_{0}, \quad g_{i}=(-1)^{i} g_{\mathrm{c}}\left(A_{\mathrm{c}}^{\ell-i} \cdot, \cdot\right)
$$

which completely agrees with the formula (3.6) for the reduced metric $g_{Q}$. Here the first term corresponds to the metric $h$ and the remaining terms represent the other block, i.e., the metric $g_{\mathrm{c}}(y) \cdot \chi_{\mathrm{nc}}\left(A_{\mathrm{c}}(y)\right)$ which can be understood as a family $g_{\mu}=\sum \mu_{i} g_{i}+g_{0}$ of metrics on $S$ parametrised by the coefficients $\mu_{1}, \ldots, \mu_{\ell}$ of the characteristic polynomial $\chi_{\mathrm{nc}}=\chi_{L}$ of the "nonconstant" block $L$. Notice that the splitting of $Q$ into the direct product $U \times S$ in both cases is determined by the decomposition of $T_{p} Q$ into two $A_{Q}$-invariant subspaces corresponding to the partition of the spectrum of $A_{Q}$ into two parts, "constant" and "non-constant". Also notice that in the both cases $\mu_{i}$ are the same: these are the elementary symmetric polynomials of non-constant eigenvalues of $L$ (or, which is the same, of $A$ ).

Formula (4.2) describes, however, a more general situation than (3.3). In particular, in Proposition 4.1, the non-constant eigenvalues may have arbitrary multiplicities and the "constant" block $\left(S, g_{\mathrm{c}}, A_{\mathrm{c}}\right)$ carries no Kähler structure. Thus, some additional specific properties of $g_{Q}$ and $A_{Q}$ should be taken into account. In particular, we need local formulas for the metric which simultaneously satisfies (4.2) and (3.3).

As we know from Lemma $2.2(1)$, the multiplicities of the non-constant eigenvalues $\rho_{1}, \ldots, \rho_{\ell}$ of $A_{Q}$ equal one and moreover $\mathrm{d} \rho_{i} \neq 0$ on $Q$. This condition guaranties that both the eigenvalues $\rho_{1}, \ldots, \rho_{\ell}$ and the symmetric polynomials $\mu_{1}, \ldots, \mu_{\ell}$ can be taken as local coordinates on $U$. Also we know from (3.3) that $S$ is endowed with a natural complex structure $J_{S}$ and for each $\mu \in U$, the metric

$$
g_{\mu}=\sum_{i=1}^{\ell} \mu_{i} g_{i}+g_{0}
$$

on $S$ is Kähler and $A_{\mathrm{c}}$ on $S$ is hermitian w.r.t. $\left(g_{\mu}, J_{S}\right)$. In addition $A_{\mathrm{c}}$ is parallel w.r.t. $g_{\mathrm{c}}=$ $g_{\mu}\left(\chi_{\mathrm{nc}}\left(A_{\mathrm{c}}\right)^{-1} \cdot, \cdot\right)$ by Proposition 4.1. This obviously implies that the metrics $g_{\mathrm{c}}$ and $g_{\mu}$ are affinely equivalent for each $\mu$, i.e., their Levi-Civita connections coincide. Hence, if we introduce $\omega_{\mathrm{c}}=$ $g_{\mathrm{c}}\left(J_{S} \cdot, \cdot\right)=\omega_{\mu}\left(\left(\chi_{\mathrm{nc}}\left(A_{\mathrm{c}}\right)\right)^{-1} \cdot, \cdot\right)$, then $\omega_{\mathrm{c}}$ is parallel and therefore $\left(g_{\mathrm{c}}, \omega_{\mathrm{c}}, J_{S}\right)$ is a Kähler structure on $S$ admitting a parallel hermitian endomorphism $A_{\mathrm{c}}$ (in other words the conclusion about the constant block in Proposition 4.1 now holds in the Kähler setting). 
Summarizing, we see that the pair $\left(g_{Q}, A_{Q}\right)$ admits the following local description.

Proposition 4.2. Using the natural decomposition $Q=U\left(x_{1}, \ldots, x_{\ell}\right) \times S\left(y_{1}, \ldots, y_{2 k}\right)$, we can write $g_{Q}$ and $A_{Q}$ as follows

$$
A_{Q}(x, y)=\left(\begin{array}{cc}
L(x) & 0 \\
0 & A_{\mathrm{c}}(y)
\end{array}\right) \quad \text { and } \quad g_{Q}(x, y)=\left(\begin{array}{cc}
h(x) & 0 \\
0 & g_{\mathrm{c}}(y) \cdot \chi_{L}\left(A_{\mathrm{c}}\right)
\end{array}\right)
$$

where

- $(L, h)$ is a compatible pair on $U$ (in the projective sense) such that the eigenvalues $\rho_{1}, \ldots, \rho_{\ell}$ of $L$ are all distinct and $\mathrm{d} \rho_{i} \neq 0$. Moreover, $\chi_{L}(t)=\operatorname{det}(t \cdot \operatorname{Id}-L)$ denotes the characteristic polynomial of $L$;

- $\left(S, g_{\mathrm{c}}, J_{S}\right)$ is a Kähler manifold and $A_{\mathrm{c}}$ is a parallel hermitian endomorphism on $S$.

The metric $h=\sum_{i, j} B_{i j} \mathrm{~d} x_{i} \mathrm{~d} x_{j}$ can be rewritten in coordinates $\mu_{1}, \ldots, \mu_{\ell}$

$$
h=\sum_{i, j=1}^{\ell} B_{i j} \mathrm{~d} x_{i} \mathrm{~d} x_{j}=\sum_{\alpha, \beta=1}^{\ell} G_{\alpha \beta} \mathrm{d} \mu_{\alpha} \mathrm{d} \mu_{\beta}, \quad B_{i j}=\sum_{\alpha, \beta=1}^{\ell} G_{\alpha \beta} \frac{\partial \mu_{\alpha}}{\partial x_{i}} \frac{\partial \mu_{\beta}}{\partial x_{j}}
$$

as in Proposition 3.2. As we know from this proposition, the components $G_{i j}$ must satisfy one additional condition, namely $\frac{\partial G_{i j}}{\partial \mu_{k}}=\frac{\partial G_{k j}}{\partial \mu_{i}}$. It turns out (see Proposition 4.4 and Lemma 4.5 below) that this property follows automatically from the compatibility of $h$ and $L$. This means that we have no more restrictions onto the reduced pair $g_{Q}$ and $A_{Q}$, and can now summarize the above discussion as follows.

Proposition 4.3. Let $g, \omega$ and $A$ be as above. Then in a neighbourhood of a regular point $p \in M^{0}$ we can introduce a local coordinate system

$$
V\left(t_{1}, \ldots, t_{\ell}\right) \times U\left(x_{1}, \ldots, x_{\ell}\right) \times S\left(y_{1}, \ldots, y_{2 k}\right)
$$

in which $g, \omega$ and $A$ take the following form

$$
\begin{gathered}
g=\sum_{\alpha, \beta=1}^{\ell} H_{\alpha \beta} \theta_{\alpha} \theta_{\beta}+\sum_{i, j=1}^{\ell} B_{i j} \mathrm{~d} x_{i} \mathrm{~d} x_{j}+\sum_{i=0}^{\ell} \mu_{i} \cdot(-1)^{i} g_{\mathrm{c}}\left(A_{\mathrm{c}}^{\ell-i} \cdot, \cdot\right) \\
\omega=\sum_{\alpha=1}^{\ell} \mathrm{d} \mu_{\alpha} \wedge \theta_{\alpha}+\sum_{i=0}^{\ell} \mu_{i} \cdot(-1)^{i} \omega_{\mathrm{c}}\left(A_{\mathrm{c}}^{\ell-i} \cdot, \cdot\right) \\
A=\sum_{\alpha, \beta=1}^{\ell} M_{\alpha}^{\beta}(x) \theta_{\beta} \otimes \frac{\partial}{\partial t_{\alpha}}+\sum_{i, j=1}^{\ell} L_{j}^{i}(x) \mathrm{d} x_{j} \otimes \frac{\partial}{\partial x_{i}}+\sum_{p, q=1}^{2 k}\left(A_{\mathrm{c}}\right)_{p}^{q} \mathrm{~d} y_{p} \otimes\left(\frac{\partial}{\partial y_{q}}-\sum_{i=1}^{\ell} \alpha_{i q} \frac{\partial}{\partial t_{i}}\right)
\end{gathered}
$$

where the ingredients in these formulas are as follows:

(1) $\left(g_{\mathrm{c}}, \omega_{\mathrm{c}}\right)$ is a Kähler structure and $A_{\mathrm{c}}=\sum_{p, q}\left(A_{\mathrm{c}}\right)_{p}^{q} \mathrm{~d} y_{p} \otimes \partial_{y_{q}}$ is a parallel hermitian endomorphism on $S$;

(2) $h=B_{i j}(x) \mathrm{d} x_{i} \mathrm{~d} x_{j}$ is a pseudo-Riemannian metric and $L(x)$ is an endomorphism on $U$ forming a compatible pair (in the projective sense);

(3) the eigenvalues $\rho_{1}, \ldots, \rho_{\ell}$ of $L$ are pairwise distinct and satisfy $\mathrm{d} \rho_{i} \neq 0$ on $U$; they are also different from the constant eigenvalues of $A_{\mathrm{c}}$;

(4) $\mu_{i}$ denote the elementary symmetric polynomials in $\rho_{1}, \ldots, \rho_{\ell}, i=1, \ldots, \ell$, and we set $\mu_{0}=1$

(5) $\theta_{i}=\mathrm{d} t_{i}+\alpha_{i}$, where $\alpha_{i}=\sum_{q} \alpha_{i q} \mathrm{~d} y_{q}$ is a 1-form on $S$ satisfying $\mathrm{d} \alpha_{i}=(-1)^{i} \omega_{\mathrm{c}}\left(A_{\mathrm{c}}^{\ell-i} \cdot, \cdot\right)$;

(6) and finally $H_{\alpha \beta}=\sum_{i, j} B^{i j} \frac{\partial \mu_{\alpha}}{\partial x_{i}} \frac{\partial \mu_{\beta}}{\partial x_{j}}$, where $B^{i j}$ is the inverse of $B_{i j}$ and $M_{\alpha}^{\beta}=\sum_{i, j} L_{j}^{i} \frac{\partial \mu_{\beta}}{\partial x_{i}} \frac{\partial x_{j}}{\partial \mu_{\alpha}}$.

Proof. The formulas (4.4) and (4.5) follow from the discussion above. It remains to derive formula (4.6) for $A$. First of all we note that the basis dual to the coframe $\theta_{i}, \mathrm{~d} x_{j}, \mathrm{~d} y_{q}$ is given by

$$
\frac{\partial}{\partial t_{i}}, \quad \frac{\partial}{\partial x_{j}}, \quad \frac{\partial}{\partial y_{q}}-\sum_{i=1}^{\ell} \alpha_{i q} \frac{\partial}{\partial t_{i}}
$$


We see that the reduction of $A$ given by (4.6) is indeed given by $A_{Q}$ from Proposition 4.2. It remains to show how $A$ acts on the Killing vector fields $\partial_{t_{i}}$. Formula (4.5) shows that $i_{\partial_{t_{\beta}}} \omega=-\mathrm{d} \mu_{\beta}$, hence, $\partial_{t_{\beta}}=J \operatorname{grad} \mu_{\beta}$. Using that $A$ commutes with $J$ and that $L$ is $h$-selfadjoint, we obtain

$$
A \frac{\partial}{\partial t_{\beta}}=J A\left(\operatorname{grad} \mu_{\beta}\right)=J L\left(\operatorname{grad}_{h} \mu_{\beta}\right)=\sum_{i, j, \alpha=1}^{\ell} L_{j}^{i} \frac{\partial \mu_{\beta}}{\partial x_{i}} \frac{\partial x_{j}}{\partial \mu_{\alpha}} \frac{\partial}{\partial t_{\alpha}}=\sum_{\alpha=1}^{\ell} M_{\alpha}^{\beta} \frac{\partial}{\partial t_{\alpha}} .
$$

which establishes formula (4.6).

Thus, we are lead to the situation described in Example 3 and, therefore, the second part of Theorem 1.5 is proved.

The main ingredients in the above local formulas are the pair $(h, L)$ on $U$ and the triple $\left(g_{\mathrm{c}}, \omega_{\mathrm{c}}, A_{\mathrm{c}}\right)$ on $S$. The 1 -forms $\alpha_{i}$ on $S$ are determined by $\left(\omega_{\mathrm{c}}, A_{\mathrm{c}}\right)$ only up to the transformation $\alpha_{i} \longmapsto \alpha_{i}+\mathrm{d} f_{i}$ for arbitrary functions $f_{i}$ on $S$. However, such functions define a fiberpreserving local transformation $f: M \rightarrow M, f(t, x, y)=\left(t_{1}+f_{1}(y), \ldots, t_{\ell}+f_{\ell}(y), x, y\right)$, that fulfils $f^{*} \theta_{i}=\theta_{i}+\mathrm{d} f_{i}, f^{*} \mathrm{~d} x_{j}=\mathrm{d} x_{j}$ and $f^{*} \mathrm{~d} y_{q}=\mathrm{d} y_{q}$ and pulls back the objects in Proposition 4.3 written down w.r.t. $\theta_{i}$ to the corresponding objects written down w.r.t. $\tilde{\theta}_{i}=\theta_{i}+\mathrm{d} f_{i}$. All the other ingredients appearing in the formulas of Proposition 4.3 can be uniquely reconstructed from $(h, L)$ and $\left(g_{\mathrm{c}}, \omega_{\mathrm{c}}, A_{\mathrm{c}}\right)$. However, we do not know yet whether these ingredients can be arbitrarily chosen or should, perhaps, satisfy some additional restrictions which are not mentioned in Proposition 4.3. The next section shows that there are no more restrictions and (4.4), (4.5) and (4.6) can be used for the local description of c-compatible $g$ and $A$. To that end, we only need to substitute into these formulas the local normal forms for $(h, L)$ and $\left(g_{\mathrm{c}}, \omega_{\mathrm{c}}, A_{\mathrm{c}}\right)$ which were previously found in [9] and [13] respectively.

4.2. Realisation. The purpose of this section is to prove the following result which is equivalent to the first part of Theorem 1.5.

Proposition 4.4. Let $h=\sum_{i, j=1}^{\ell} B_{i j}(x) \mathrm{d} x_{i} \mathrm{~d} x_{j}$ be a pseudo-Riemannian metric and $L(x)$ an endomorphism on $U$ forming a compatible pair (in the projective sense) and let $\left(g_{\mathrm{c}}, \omega_{\mathrm{c}}\right)$ be a Kähler structure of arbitrary signature and $A_{\mathrm{c}}$ a parallel endomorphism on $S$. Suppose that the eigenvalues of $L$ and $A_{\mathrm{c}}$ satisfy condition (3) from Proposition 4.3 and 1-forms $\alpha_{i}$ on $S$ are chosen as in condition (5). Then

- $g$ and $\omega$ given by (4.4) and (4.5) define a Kähler structure on $V \times U \times S$;

- A given by (4.6) is hermitian w.r.t. $(g, \omega)$ and satisfies $(1.4)$, in other words $A$ and $(g, \omega)$ are c-compatible.

Proof. To verify that $g$ and $\omega$ define a Kähler structure, we use Proposition 3.2. Formulas (4.4) and (4.5) are similar to (3.3) and (3.4) but we still need to verify some conditions. First of all, we can use $\mu_{1}, \ldots, \mu_{\ell}$ as local coordinates on $U$ to rewrite the term $h=\sum_{i, j} B_{i j} \mathrm{~d} x_{i} \mathrm{~d} x_{j}$ as $\sum_{\alpha, \beta} G_{\alpha \beta} \mathrm{d} \mu_{\alpha} \mathrm{d} \mu_{\beta}$, where $B_{i j}=\sum_{\alpha, \beta} G_{\alpha \beta} \frac{\partial \mu_{\alpha}}{\partial x_{i}} \frac{\partial \mu_{\beta}}{\partial x_{j}}$ and then the matrices $H_{\alpha \beta}$ and $G_{\alpha \beta}$ are inverse to each other as required in Proposition 3.2. Next we need to check that $g_{\mu}=\sum_{i=0}^{\ell} \mu_{i} \cdot(-1)^{i} g_{\mathrm{c}}\left(A_{\mathrm{c}}^{\ell-i} \cdot, \cdot\right)$ and $\omega_{\mu}=\sum_{i=0}^{\ell} \mu_{i} \cdot(-1)^{i} \omega_{\mathrm{c}}\left(A_{\mathrm{c}}^{\ell-i} \cdot, \cdot\right)$ define a Kähler structure on $S$ for any $\mu$, but this condition immediately follows from the fact that $\left(g_{\mathrm{c}}, \omega_{\mathrm{c}}\right)$ is Kähler and $A_{\mathrm{c}}$ is hermitian and parallel with respect to it. Notice that the complex structure $J_{S}$ is, by construction, the same for all $\left(g_{\mu}, \omega_{\mu}\right)$.

Less trivial is the fact that $h$ is a Hessian metric in the coordinates $\mu_{1}, \ldots, \mu_{\ell}$, i.e., that $\partial_{\mu_{i}} G_{j k}=$ $\partial_{\mu_{j}} G_{i k}$ holds for all $i, j, k$ (condition (3) from Proposition 3.2). To prove it, we first notice that this condition is equivalent to the fact that the vector fields $\operatorname{grad} \mu_{1}, \ldots, \operatorname{grad} \mu_{\ell}$ commute. Indeed, $\partial_{\mu_{i}} G_{j k}=\partial_{\mu_{j}} G_{i k}$ means that the 1-forms $\beta_{k}=\sum_{i} G_{i k} \mathrm{~d} \mu_{i}$ are all closed. Hence, the statement immediately follows from the observation that the forms $\beta_{1}, \ldots, \beta_{\ell}$ are dual to the vector fields $\operatorname{grad} \mu_{1}, \ldots, \operatorname{grad} \mu_{\ell}$, i.e., $\beta_{k}\left(\operatorname{grad} \mu_{j}\right)=\delta_{k j}$.

Thus, it remains to prove the following lemma (cf. Lemma 2.2 (6) which is a c-projective analogue of this statement). 
Lemma 4.5. Let $h$ be a pseudo-Riemannian metric on $U \subset \mathbb{R}^{\ell}$ and $L$ be an $h$-selfadjoint endomorphism compatible with $h$ in the projective sense. Let

$$
\operatorname{det}(t \cdot \operatorname{Id}-L)=\sum_{i=0}^{\ell}(-1)^{i} \mu_{i} t^{\ell-i}
$$

where the functions $\mu_{i}, i=1, \ldots, \ell$, are the elementary symmetric functions in the eigenvalues of $L$ and $\mu_{0}=1$. Then,

$$
\left[\operatorname{grad} \mu_{i}, \operatorname{grad} \mu_{j}\right]=0 \text { for all } i, j .
$$

Proof. First of all, since the vector fields $\operatorname{grad} \mu_{1}, \ldots, \operatorname{grad} \mu_{\ell}$ are constant linear combinations of the vector fields of the form $v_{t}=\operatorname{grad} \operatorname{det}(t \cdot \operatorname{Id}-L)$ for $t \in \mathbb{R}$ and vice versa, it suffices to prove that

$$
\left[v_{t}, v_{s}\right]=0
$$

for all $t, s \in \mathbb{R}$. Moreover, it suffices to prove (4.7) for $t, s$ which do not belong to the spectrum of $L$ locally in a neighbourhood of a point. For $L: T M \rightarrow T M$ an arbitrary non-degenerate endomorphism and $X$ an arbitrary vector field, recall the general formula

$$
X(\operatorname{det} L)=(\operatorname{det} L) \operatorname{tr}\left(L^{-1} \nabla_{X} L\right) .
$$

In our case, $L$ and therefore $L_{s}=s \cdot \operatorname{Id}-L$ satisfy (1.7), i.e.

$$
\nabla_{X}(s \cdot \mathrm{Id}-L)=-\nabla_{X} L=-X^{\mathrm{b}} \otimes \Lambda-\Lambda^{\mathrm{b}} \otimes X
$$

holds for a certain vector field $\Lambda$. Defining $f_{s}=\operatorname{det} L_{s}$ and combining the previous two equations we obtain

$$
X\left(f_{s}\right)=-2 f_{s} h\left(X, L_{s}^{-1} \Lambda\right) .
$$

or equivalently, $v_{s}=\operatorname{grad} f_{s}=-2 f_{s} L_{s}^{-1} \Lambda$. Note that this formula is meaningful and holds true even if $s$ is in the spectrum of $L$. We calculate

$$
\nabla_{X} v_{s}=2 f_{s}\left[h\left(X, L_{s}^{-1} \Lambda\right) L_{s}^{-1} \Lambda-h\left(\Lambda, L_{s}^{-1} \Lambda\right) L_{s}^{-1} X-L_{s}^{-1} \nabla_{X} \Lambda\right] .
$$

It is a well-known statement in projective geometry that the endomorphisms $L$ and $\nabla \Lambda$ commute, see for example the discussion below Theorem 7 in [6]. Replacing $X$ by $v_{t}$ in the last equation and using $\left[L_{t}^{-1}, \nabla \Lambda\right]=0$, we obtain

$$
\nabla_{v_{t}} v_{s}=4 f_{s} f_{t}\left[-h\left(L_{t}^{-1} \Lambda, L_{s}^{-1} \Lambda\right) L_{s}^{-1} \Lambda+h\left(\Lambda, L_{s}^{-1} \Lambda\right) L_{s}^{-1} L_{t}^{-1} \Lambda+L_{s}^{-1} L_{t}^{-1} \nabla_{\Lambda} \Lambda\right] .
$$

Thus,

$$
\begin{gathered}
{\left[v_{t}, v_{s}\right]=\nabla_{v_{t}} v_{s}-\nabla_{v_{s}} v_{t}} \\
=4 f_{s} f_{t}\left[-h\left(\Lambda, L_{s}^{-1} L_{t}^{-1} \Lambda\right)\left(L_{s}^{-1}-L_{t}^{-1}\right) \Lambda+h\left(\Lambda,\left(L_{s}^{-1}-L_{t}^{-1}\right) \Lambda\right) L_{s}^{-1} L_{t}^{-1} \Lambda\right] .
\end{gathered}
$$

Inserting the identity $L_{s}^{-1}-L_{t}^{-1}=(t-s) L_{s}^{-1} L_{t}^{-1}$ into the last equation, we obtain (4.7) as we claimed.

Applying this lemma to $h$ and $L$ from Proposition 4.4, we get condition (3) from Proposition 3.2. Thus, now Proposition 3.2 implies that $g$ and $\omega$ given by (4.4) and (4.5) indeed define a Kähler structure on $V \times U \times S$ which completes the proof of the first statement of Proposition 4.4.

It is easy to see that $A$ is hermitian w.r.t. $(g, \omega)$. It remains to show that $A$ satisfies $(1.4)$ and we will proceed as follows. Consider the hermitian metric

$$
\hat{g}=\left(\operatorname{det}_{\mathbb{C}} A\right)^{-1} g\left(A^{-1} \cdot, \cdot\right)
$$

obtained from $g$ and $A$ by solving (1.2) w.r.t. $\hat{g}$. First, we show that $\hat{g}$ is a Kähler metric on $(M, J)$. Then we show that $g$ and $\hat{g}$ are c-projectively equivalent. This implies that $A$ satisfies (1.4) and we are done.

Proposition 4.6. The metric $\hat{g}$ is a Kähler metric on $(M, J)$. 
Proof. We use the (local) formulas for $g, \omega$ and $A$ from Proposition 4.3 and the notation introduced there. Since $\hat{g}$ is hermitian w.r.t. $J$ by construction, we only need to check that $\hat{\omega}=\hat{g}(J \cdot, \cdot)=$ $\left(\operatorname{det}_{\mathbb{C}} A\right)^{-1} \omega\left(A^{-1} \cdot, \cdot\right)$ is closed.

Notice that $\operatorname{det}_{\mathbb{C}} A=c \cdot \mu_{\ell}$ for some constant $c$ so that we may, without loss of generality, replace $\hat{\omega}$ by $\mu_{\ell}^{-1} \omega\left(A^{-1} \cdot, \cdot\right)$.

Then, by using (4.5) and (4.6), we get

$$
c \cdot \hat{\omega}=\sum_{i=1}^{\ell} \mu_{\ell}^{-1}\left(\mathrm{~d} \mu_{i} \circ L^{-1}\right) \wedge \theta_{i}+\sum_{k=0}^{\ell} \frac{\mu_{k}}{\mu_{\ell}} \omega_{k}\left(A_{\mathrm{c}}^{-1} \cdot, \cdot\right),
$$

where $\omega_{k}=(-1)^{k} \omega_{\mathrm{c}}\left(A_{\mathrm{c}}^{\ell-k} \cdot, \cdot\right)$.

The closeness of $\hat{\omega}$ now follows from two facts

- $\mu_{\ell}^{-1}\left(\mathrm{~d} \mu_{i} \circ L^{-1}\right)=-\mathrm{d} \hat{\mu}_{\ell+1-i}$, where $\hat{\mu}_{k}$ denotes the $k$ th elementary symmetric polynomial in the eigenvalues of $L^{-1}$, i.e. in $\rho_{1}^{-1}, \ldots, \rho_{\ell}^{-1}$. This is a general property of a compatible pair $(h, L)$, see Lemma 4.7 below.

- $\sum_{k=0}^{\ell} \frac{\mu_{k}}{\mu_{\ell}} \omega_{k}\left(A_{\mathrm{c}}^{-1} \cdot, \cdot\right)=\sum_{k=0}^{\ell} \hat{\mu}_{\ell-k} \omega_{k+1}=\sum_{i=1}^{\ell} \hat{\mu}_{\ell+1-i} \omega_{i}+\omega_{\ell+1}$. This relation is straightforward.

Hence

$$
c \cdot \hat{\omega}=\sum_{i=1}^{\ell} \mathrm{d} \hat{\mu}_{\ell+1-i} \wedge \theta_{i}+\sum_{i=1}^{\ell} \hat{\mu}_{\ell+1-i} \omega_{i}+\omega_{\ell+1}
$$

and the property $\mathrm{d} \hat{\omega}=0$ becomes obvious, as $\omega_{k}$ 's are all closed and $\mathrm{d} \theta_{i}=\omega_{i}$ by construction.

Thus, in order to complete the proof of Proposition 4.6 it remains to prove

Lemma 4.7. Let $h$ and $L$ be compatible in the projective sense. Then the following relation holds

$$
\frac{1}{\operatorname{det} L}\left(\mathrm{~d} \mu_{i} \circ L^{-1}\right)=-\mathrm{d} \hat{\mu}_{\ell+1-i}
$$

for $i=1, \ldots, \ell$, where $\hat{\mu}_{k}$ is the $k$ th symmetric polynomial in $\rho_{1}^{-1}, \ldots, \rho_{\ell}^{-1}$.

Proof. Recall that the compatibility condition (1.7) implies that the Nijenhuis torsion of $L$ vanishes (see for instance [7, Theorem 1]). Lemma 10 from [8] states that for such $L$ the following formula holds:

$$
\mathrm{d} \chi_{L}(t) \circ L-t \cdot \mathrm{d} \chi_{L}(t)=\chi_{L}(t) \cdot \mathrm{d} \operatorname{tr} L
$$

where $\chi_{L}(t)=\operatorname{det}(t \cdot \operatorname{Id}-L)$ is the characteristic polynomial of $L$. Let us multiply the both sides of this formula by $L^{-1}$ :

$$
\mathrm{d} \chi_{L}(t)-t \cdot \mathrm{d} \chi_{L}(t) \circ L^{-1}=\chi_{L}(t) \cdot \mathrm{d} \operatorname{tr} L \circ L^{-1}
$$

Hence,

$$
\mathrm{d} \chi_{L}(t) \circ L^{-1}=\frac{1}{t}\left(\mathrm{~d} \chi_{L}(t)-\chi_{L}(t) \cdot \operatorname{dtr} L \circ L^{-1}\right)
$$

Using another nice formula $\operatorname{dtr} L \circ L^{-1}=\mathrm{d}(\ln \operatorname{det} L)$, we get

$$
\begin{gathered}
\mathrm{d} \chi_{L}(t) \circ L^{-1}=\frac{1}{t}\left(\mathrm{~d} \chi_{L}(t)-\chi_{L}(t) \cdot \mathrm{d}(\ln \operatorname{det} L)\right)=\frac{1}{t} \frac{\operatorname{det} L \cdot \mathrm{d} \chi_{L}(t)-\chi_{L}(t) \cdot \mathrm{d} \operatorname{det} L}{\operatorname{det} L}= \\
\frac{\operatorname{det} L}{t} \frac{\operatorname{det} L \cdot \mathrm{d} \chi_{L}(t)-\chi_{L}(t) \cdot \mathrm{d} \operatorname{det} L}{\operatorname{det}^{2} L}=\frac{\operatorname{det} L}{t} \cdot \mathrm{d}\left(\frac{\chi_{L}(t)}{\operatorname{det} L}\right),
\end{gathered}
$$

or equivalently

$$
\frac{1}{\operatorname{det} L} \mathrm{~d} \chi_{L}(t) \circ L^{-1}=t^{-1} \mathrm{~d}\left(\frac{\chi_{L}(t)}{\operatorname{det} L}\right),
$$

which coincide with the desired relation if we take into account that $\chi_{L}(t)=\sum_{i=0}^{\ell}(-1)^{i} \mu_{i} t^{\ell-i}$ and consider $t$ as a formal parameter. 
Remark 4.1. We can derive the formulas in Lemma 4.7 in an alternative way: by the same arguments as used in the proof of Lemma $2.2(7)$, one easily derives the formula

$$
\operatorname{grad}_{\hat{h}} \chi_{L^{-1}}(1 / t)=\frac{(-1)^{\ell}}{t^{\ell-1}} \operatorname{grad}_{h} \chi_{L}(t),
$$

where $\hat{h}$ is the metric given by (1.8). The only thing we used to derive (4.11) is that $\operatorname{grad}_{h} \rho_{i}$ is in the $\rho_{i}$-eigenspace of $L$ for each eigenvalue $\rho_{i}$ of $L$ (and, of course, that each $\rho_{i}$ is smooth with $\mathrm{d} \rho_{i} \neq 0$ ). The polynomial expression (4.11) in $t$ resp. $1 / t$ gives rise to equivalent equations on the coefficients. These equations are given by $\operatorname{grad}_{h} \mu_{i}=-\operatorname{grad}_{\hat{h}} \hat{\mu}_{\ell+1-i}$ (or, what is equivalent, $\operatorname{grad}_{\hat{h}} \hat{\mu}_{i}=-\operatorname{grad}_{h} \mu_{\ell+1-i}$ ) for all $i$. Taking into account formula (1.8) for $\hat{h}$ and the fact that $L$ is $h$-selfadjoint, one sees that the latter equations are just the gradient version of the formulas in Lemma 4.7 .

Thus, $\hat{g}$ defined by (4.10) is a Kähler metric on $(M, J)$.

Consider now the Kähler metrics $g, \hat{g}$ on $(M, J)$ with Levi-Civita connections $\nabla, \hat{\nabla}$ respectively. Let $T$ be the (1,2)-tensor defined by

$$
T(X, Y)=\hat{\nabla}_{X} Y-\nabla_{X} Y .
$$

Since $\nabla, \hat{\nabla}$ are both torsion-free, $T$ is symmetric in $X, Y$. Moreover, since both $\nabla, \hat{\nabla}$ preserve $J$, we have the symmetry

$$
T(X, J Y)=T(J X, Y)=J T(X, Y) .
$$

Lemma 4.8. The tensor $T$ satisfies

$$
T(X, Y)=\Phi(X) Y+\Phi(Y) X-\Phi(J X) J Y-\Phi(J Y) J X .
$$

for a certain 1-form $\Phi$ on $M$.

Proof. First we recall that $A$ preserves the vertical distribution $\mathcal{V}$ and the horizontal distribution $\mathcal{Q}=\mathcal{V}^{\perp}$ so that $\mathcal{Q}$ does not change if we consider $\hat{g}$ instead of $g$. We will use the same symbol for a vector field on $Q$ and its horizontal lift to $M$.

Denoting by $\operatorname{pr}_{\mathcal{Q}}: T M \rightarrow \mathcal{Q}$ the projection to the horizontal distribution, the O'Neill formula (3.7) implies

$$
\operatorname{pr}_{\mathcal{Q}}(T(X, Y))=\hat{\nabla}_{X}^{Q} Y-\nabla_{X}^{Q} Y
$$

for vector fields $X, Y \in \Gamma(T Q)$, where $\nabla^{Q}, \hat{\nabla}^{Q}$ are the Levi-Civita connections of the horizontal parts $g_{Q}$ and $\hat{g}_{Q}$ of $g$ and $\hat{g}$ respectively.

From formula (4.10) we see that $\hat{g}_{Q}=c \cdot\left(\operatorname{det} A_{Q}\right)^{-1} g_{Q}\left(A_{Q}^{-1} \cdot, \cdot\right)$ for some constant $c$, where $A_{Q}$ is the quotient of $A$ and we used that $\operatorname{det}_{\mathbb{C}} A$ equals $\operatorname{det} A_{Q}$ up to multiplying with a constant. Comparing this formula for $\hat{g}_{Q}$ with (1.8) and noting that, by construction, $g_{Q}$ and $A_{Q}$ are compatible on $Q$, we see that $\hat{g}_{Q}$ is projectively equivalent to $g_{Q}$. Thus, we have that

$$
\hat{\nabla}_{X}^{Q} Y-\nabla_{X}^{Q} Y=\Phi(X) Y+\Phi(Y) X
$$

is satisfied for all $X, Y \in \Gamma(Q)$ for a 1-form $\Phi$ on $Q$. Indeed, the fact that (4.14) is equivalent to $g_{Q}, \hat{g}_{Q}$ being projectively equivalent is a classical statement in projective geometry, see [24].

Using (4.14), we obtain that the horizontal part of $T$ is given by

$$
\operatorname{pr}_{\mathcal{Q}}(T(X, Y))=\Phi(X) Y+\Phi(Y) X .
$$

However, since $\mathcal{V}$ is spanned by the Killing vector fields $K_{i}$, any $g$ - or $\hat{g}$-geodesic $\gamma(t)$ in $M$ being initially tangent to $\mathcal{Q}$ remains tangent to it for all values of $t$. It follows that $\nabla_{X} X, \hat{\nabla}_{X} X \in \mathcal{Q}$ whenever $X \in \mathcal{Q}$. Then $T(X, X)$ in $\mathcal{Q}$ for all $X \in \mathcal{Q}$ and by polarisation (recall that $T$ is symmetric) we have $T(X, Y) \in \mathcal{Q}$ for all $X, Y \in \mathcal{Q}$. Thus, we obtain

$$
T(X, Y)=\Phi(X) Y+\Phi(Y) X .
$$

for all $X, Y \in \mathcal{Q}$. Since the form $\Phi$ in (4.14) is explicitly given by the formula

$$
\Phi=-\frac{1}{2} \mathrm{~d} \ln (\operatorname{det} L)
$$


(which is a classical formula that can be obtained from (4.14) by contraction), we see that it vanishes upon insertion of vector fields that are contained in the generalised eigenspaces of $A$ corresponding to constant eigenvalues. Thus, (4.15) establishes formula (4.13) for vector fields tangent to $\mathcal{Q}$.

It remains to verify equation (4.13) upon insertion of vertical vector fields $J X, J Y$, where $X, Y \in J \mathcal{V} \subseteq \mathcal{Q}$. Using (4.12), we obtain

$$
\begin{gathered}
T(J X, J Y)=-T(X, Y) \stackrel{(4.15)}{=}-\Phi(X) Y-\Phi(Y) X \\
=\underbrace{\Phi(J X) J Y+\Phi(J Y) J X}_{=0}-\Phi(J J X) J J Y-\Phi(J J Y) J J X
\end{gathered}
$$

which establishes (4.13) evaluated on $J X, J Y$. Further, for arbitrary $Z$ tangent to $\mathcal{Q}$, we obtain

$$
\begin{gathered}
T(Z, J X)=J T(Z, X) \stackrel{(4.15)}{=} \Phi(Z) J X+\Phi(X) J Z \\
=\Phi(Z) J X+\underbrace{\Phi(J X)}_{=0} Z-\underbrace{\Phi(J Z)}_{=0} J J X-\Phi(J J X) J Z
\end{gathered}
$$

establishing (4.13) when evaluated on $Z, J X$. Thus, we verified (4.13) on all possible combinations of tangent vectors and the claim follows.

It is a classical statement in c-projective geometry, see for example [32, 40], and we used this fact already in the proof of Lemma 2.1 that two complex torsion-free connections $\nabla, \hat{\nabla}$ on a complex manifold $(M, J)$ are c-projectively equivalent (i.e. their $J$-planar curves coincide) if and only if (4.13) is satisfied for a certain 1-form $\Phi$. Lemma 4.8 then shows that $g, \hat{g}$ are c-projectively equivalent. This implies that $A=A(g, \hat{g})$ is a solution of equation (1.4) and completes the proof of the second part of Proposition 4.4.

4.3. Explicit formulas. In the preceding sections, we have proved Theorem 1.5 (see Propositions 4.3 and 4.4) which can be understood as an invariant version of Theorem 1.6. We are now going to derive the formulas from Example 5. Our starting point is Proposition 4.3. We will derive explicit formulas for all the objects that have been introduced there and thereby prove Theorem 1.6.

The compatible pair $h, L$ can be described explicitly by using the results from [9]. The latter article contains explicit formulas for a compatible pair in the general pseudo-Riemannian case. In our case, there are no Jordan blocks (with non-constant eigenvalues) and the formulas become similar to the classical Levi-Civita theorem - the only modification being signs $\varepsilon_{i}= \pm 1$ for each non-constant real eigenvalue $\rho_{i}$ (which allow us to "produce" an arbitrary signature) and the occurrence of complex eigenvalues. Let

$$
E_{\mathrm{nc}}=\left\{\rho_{1}, \bar{\rho}_{1}, \ldots, \rho_{r}, \bar{\rho}_{r}, \rho_{r+1}, \ldots, \rho_{r+q}\right\}
$$

denote the set of (non-constant) eigenvalues of $L$ ( $r$ pairs of complex-conjugate eigenvalues and $q$ real eigenvalues). Recall that the "gluing data" in [9, Theorem 1.3] takes the form of a 1-dimensional block

$$
h_{i}=\varepsilon_{i} \mathrm{~d} x_{i}^{2}, \quad L_{i}=\rho_{i}\left(x_{i}\right) \partial_{x_{i}} \otimes \mathrm{d} x_{i},
$$

for a real eigenvalue $\rho_{i}$ and (as follows from [9, Theorems 5] or [11, Theorem 2]) the form of a 2-dimensional block

$$
h_{i}=\frac{1}{4}\left(\bar{\rho}_{i}\left(\bar{z}_{i}\right)-\rho_{i}\left(z_{i}\right)\right)\left(\mathrm{d} z_{i}^{2}-\mathrm{d} \bar{z}_{i}^{2}\right), \quad L_{i}=\rho_{i}\left(z_{i}\right) \partial_{z_{i}} \otimes \mathrm{d} z_{i}+\bar{\rho}_{i}\left(\bar{z}_{i}\right) \partial_{\bar{z}_{i}} \otimes \mathrm{d} \bar{z}_{i},
$$

if $\rho_{i}, \bar{\rho}_{i}$ is a pair of complex conjugate eigenvalues, where $z_{j}$ is a complex coordinate w.r.t. which $\rho_{j}$ is a holomorphic function. 
Thus, by [9, Theorem 1.3], we find local coordinates $z_{1}, \ldots, z_{r}, x_{r+1}, \ldots, x_{r+q}{ }^{3}$ (where the $z_{j}$ are complex coordinates and the $x_{j}$ are real coordinates) such that

$$
\begin{gathered}
h=-\frac{1}{4} \sum_{i=1}^{r}\left(\Delta_{i} \mathrm{~d} z_{i}^{2}+c . c .\right)+\sum_{i=r+1}^{r+q} \Delta_{i} \varepsilon_{i} \mathrm{~d} x_{i}^{2}, \\
L=\sum_{i=1}^{r}\left(\rho_{i} \partial_{z_{i}} \otimes \mathrm{d} z_{i}+\text { c.c. }\right)+\sum_{i=r+1}^{r+q} \rho_{i} \partial_{x_{i}} \otimes \mathrm{d} x_{i},
\end{gathered}
$$

where for $1 \leq i \leq r, \rho_{i}\left(z_{i}\right)$ is a holomorphic function of $z_{i}$, for $r+1 \leq i \leq r+q, \rho_{i}\left(x_{i}\right)$ only depends on $x_{i}$, "c.c." denotes the complex conjugate of the preceding term and

$$
\Delta_{i}=\prod_{\rho \in E_{\mathrm{nc}} \backslash\left\{\rho_{i}\right\}}\left(\rho_{i}-\rho\right)
$$

The parts of $g, \omega$ and $A$ in (1.16) and (1.17), which correspond to the "constant" block, are obtained from the expressions

$$
\sum_{i=0}^{\ell} \mu_{i} \cdot(-1)^{i} g_{\mathrm{c}}\left(A_{\mathrm{c}}^{\ell-i} \cdot, \cdot\right), \sum_{i=0}^{\ell} \mu_{i} \cdot(-1)^{i} \omega_{\mathrm{c}}\left(A_{\mathrm{c}}^{\ell-i} \cdot, \cdot\right) \text { and } \sum_{p, q=1}^{2 k}\left(A_{\mathrm{c}}\right)_{p}^{q} \mathrm{~d} y_{p} \otimes\left(\frac{\partial}{\partial y_{q}}-\sum_{i=1}^{\ell} \alpha_{i q} \frac{\partial}{\partial t_{i}}\right)
$$

in the formulas $(4.4),(4.5)$ and $(4.6)$ by decomposing $\left(g_{\mathrm{c}}, \omega_{\mathrm{c}}\right)$ in the sense of de Rham-Wu [15, 41] according to the parallel distributions belonging to the generalised eigenspaces of $A_{\mathrm{c}}$. Each component $\left(g_{\gamma}, \omega_{\gamma}, A_{\gamma}\right)$ of this decomposition, as we have already mentioned, can be described explicitly using the results of Boubel [13].

To establish the formulas (1.16) and (1.17) for $g, \omega$ and $A$, it remains to find the formulas for the parts of $g, \omega$ and $A$ in the direction tangent to the Killing vector fields. We will specialise the general coordinate system $x_{1}, \ldots, x_{\ell}$ in formula (4.4) by choosing $\mu_{1}, \ldots, \mu_{\ell}$ as coordinates, compare also the formulas (3.3)-(3.5) in Proposition 3.2. With this choice of coordinates, the matrix $H_{i j}$ (inverse to $G_{i j}$ defined by $h=G_{i j} \mathrm{~d} \mu_{i} \mathrm{~d} \mu_{j}$ ) is just given by

$$
H_{i j}=g\left(K_{i}, K_{j}\right)=g\left(\operatorname{grad}_{g} \mu_{i}, \operatorname{grad}_{g} \mu_{j}\right)=h\left(\operatorname{grad}_{h} \mu_{i}, \operatorname{grad}_{h} \mu_{j}\right) .
$$

Let $\mu_{i}(\hat{\rho})$ denote the $i$ th elementary symmetric polynomial in the $\ell-1$ variables $E_{\mathrm{nc}} \backslash\{\rho\}$. Writing $\mathrm{d} \mu_{i}=\sum_{\rho \in E_{\mathrm{nc}}} \mu_{i-1}(\hat{\rho}) \mathrm{d} \rho$ in the coordinates from formula (4.16) we have

$$
\mathrm{d} \mu_{i}=\sum_{s=1}^{r}\left(\mu_{i-1}\left(\hat{\rho_{s}}\right) \frac{\partial \rho_{s}}{\partial z_{s}} \mathrm{~d} z_{s}+\text { c.c. }\right)+\sum_{s=r+1}^{r+q} \mu_{i-1}\left(\hat{\rho}_{s}\right) \frac{\partial \rho_{s}}{\partial x_{s}} \mathrm{~d} x_{s} .
$$

Using the formula (4.16) for $h$ together with (4.18), we obtain

$$
\operatorname{grad}_{h} \mu_{j}=-4 \sum_{s=1}^{r}\left(\frac{\mu_{j-1}\left(\hat{\rho_{s}}\right)}{\Delta_{s}} \frac{\partial \rho_{s}}{\partial z_{s}} \frac{\partial}{\partial z_{s}}+c . c .\right)+\sum_{s=r+1}^{r+q} \varepsilon_{s} \frac{\mu_{j-1}\left(\hat{\rho}_{s}\right)}{\Delta_{s}} \frac{\partial \rho_{s}}{\partial x_{s}} \frac{\partial}{\partial x_{s}} .
$$

From (4.17), (4.18) and (4.19), we obtain

$$
H_{i j}=-4 \sum_{s=1}^{r}\left(\frac{\mu_{i-1}\left(\hat{\rho_{s}}\right) \mu_{j-1}\left(\hat{\rho_{s}}\right)}{\Delta_{s}}\left(\frac{\partial \rho_{s}}{\partial z_{s}}\right)^{2}+c . c .\right)+\sum_{s=r+1}^{r+q} \varepsilon_{s} \frac{\mu_{i-1}\left(\hat{\rho}_{s}\right) \mu_{j-1}\left(\hat{\rho}_{s}\right)}{\Delta_{s}}\left(\frac{\partial \rho_{s}}{\partial x_{s}}\right)^{2} .
$$

Inserting the formulas (4.16) and (4.20) into (4.4), we obtain the formula (1.16) for $g$. It remains to find the formula for $A$ and the formulas for $J$ acting on $\theta_{i}$ and $\mathrm{d} \rho_{j}$ respectively.

Taking the differential of the identity

$$
\prod_{\rho \in E_{\mathrm{nc}}}(t-\rho)=\sum_{s=0}^{\ell}(-1)^{s} \mu_{s} t^{\ell-s}
$$

\footnotetext{
${ }^{3}$ These coordinates should note be confused with the general coordinate system $x_{1}, \ldots, x_{\ell}$ used, for instance, in Proposition 4.3.
} 
and inserting $t=\rho_{i}$, we obtain

$$
\mathrm{d} \rho_{i}=\frac{1}{\Delta_{i}} \sum_{s=1}^{\ell}(-1)^{s-1} \rho_{i}^{\ell-s} \mathrm{~d} \mu_{s}
$$

By (3.5), we have

$$
\mathrm{d} \rho_{i} \circ J=-\frac{1}{\Delta_{i}} \sum_{s, t=1}^{\ell}(-1)^{s-1} \rho_{i}^{\ell-s} H_{s t} \theta_{t} .
$$

Inserting (4.20) into this and applying standard Vandermonde identities (see the Appendix of [1]), we obtain

$$
\mathrm{d} z_{i} \circ J=4 \frac{1}{\Delta_{i}} \frac{\partial \rho_{i}}{\partial z_{i}} \sum_{j=1}^{\ell} \mu_{j-1}\left(\hat{\rho}_{i}\right) \theta_{j} \text { for } 1 \leq i \leq r
$$

and

$$
\mathrm{d} x_{i} \circ J=-\frac{\varepsilon_{i}}{\Delta_{i}} \frac{\partial \rho_{i}}{\partial x_{i}} \sum_{j=1}^{\ell} \mu_{j-1}\left(\hat{\rho}_{i}\right) \theta_{j} \text { for } r+1 \leq i \leq r+q .
$$

Inverting these formulas by using Vandermonde identities shows the formula for $\theta_{i} \circ J$ expressed as a linear combination of the $\mathrm{d} z_{i}$ and $\mathrm{d} x_{i}$. This establishes the formula for $J$ in (1.16).

Let us derive the remaining part of the formula (1.17) for $A$. The formula for $\omega$ in Proposition 4.3 shows that the Killing vector fields $\frac{\partial}{\partial t_{i}}$ coincide with $K_{i}=J \operatorname{grad} \mu_{i}$. We show

$$
A K_{i}=\mu_{i} K_{1}-K_{i+1} \text { for all } i=1, \ldots, \ell
$$

(where we put $K_{\ell+1}=0$ ) which immediately implies (1.17). Hence, as soon as (4.21) is derived, all formulas from Example 5 are established and Theorem 1.6 is proven.

Formula (4.21) is in fact the reformulation to our setting of [1, formula (58)] and it can be proven in the same way: let $v_{t}=\operatorname{grad}_{h} \chi_{L}(t)$, where as usual $\chi_{L}(t)=\operatorname{det}(t \cdot \mathrm{Id}-L)=\sum_{i=0}^{\ell}(-1)^{i} \mu_{i} t^{\ell-i}$ denotes the characteristic polynomial of $L$ (in the terminology of Proposition 4.3). For abbreviation define $v_{i}=\operatorname{grad}_{h} \mu_{i}$ such that $K_{i}=J v_{i}$ and $2 \Lambda=v_{1}$. Recall that, using the compatibility of $L$ and the metric $h$, we have derived the identity

$$
(t \cdot \operatorname{Id}-L) v_{t}=-\operatorname{det}(t \cdot \operatorname{Id}-L) v_{1}=-\sum_{i=0}^{\ell}(-1)^{i} \mu_{i} t^{\ell-i} v_{1}
$$

in the proof of Lemma 4.5. Inserting $v_{t}=\sum_{i=1}^{\ell}(-1)^{i} t^{\ell-i} v_{i}$ into the left-hand side of $(4.22)$ and setting $v_{\ell+1}=0$, we obtain $0=\sum_{i=1}^{\ell}(-1)^{i}\left(v_{i+1}+L v_{i}-\mu_{i} v_{1}\right) t^{\ell-i}$, hence, $L v_{i}=\mu_{i} v_{1}-v_{i+1}$. Of course, this holds also with $L$ replaced by $A$ and (4.21) follows after multiplying with $J$ and using that $A$ commutes with $J$.

\section{Proof of the Yano-Obata conjecture (Theorem 1.1)}

The main goal of this section is to prove Theorem 1.1. Simultaneously, we will give a proof of an important special case of the projective Lichnerowicz conjecture (Theorem 1.2), see Theorem 5.1.

Recall that the existence of a projective vector field $v$ for a (pseudo)-Riemannian metric $g$ implies the existence of an endomorphism $A$ compatible with $g$. According to Proposition 4.1, in a neighbourhood of a regular point, $A$ naturally splits into two blocks $A_{\mathrm{c}}$ and $L$ with constant and non-constant eigenvalues respectively.

If the non-constant block $L$ (see Proposition 4.1) is diagonalisable ${ }^{4}$, i.e. contains no Jordan blocks, then the proofs of Theorems 1.1 and 1.2 are almost identical. For that reason, parallel to the proof of the Yano-Obata conjecture, we will prove the following version of the (pseudoRiemannian) projective Lichnerowicz conjecture:

\footnotetext{
${ }^{4}$ Recall that in the c-projective setting this condition is fulfilled automatically (see Lemma 2.2 (1)), whereas in the projective setting non-constant Jordan blocks are allowed.
} 
Theorem 5.1. Let $M$ be a closed connected manifold with an indefinite metric $g$ on it. Assume that $(M, g)$ admits a projective vector field $v$ and let $A$ be an endomorphism compatible with $g$ in the projective sense, $A \neq c \cdot \mathrm{Id}$. If there exists a regular point $p \in M^{0}$ at which the non-constant block of $A$ is diagonalisable, then $v$ is affine.

In other words, this theorem says that in the absence of Jordan blocks with non-constant eigenvalues, the (pseudo-Riemannian) projective Lichnerowicz conjecture holds true, i.e., nonaffine projective vector fields do not exist on compact manifolds with indefinite metrics. To complete the proof of this conjecture in full generality, it remains to show that Jordan blocks with non-constant eigenvalues are also "forbidden". For Lorentzian metrics, this will be done in Section 6 which is the final step of the proof of Theorem 1.2.

The proof of Theorem 5.1 is organised as a series of remarks: at any step of the proof of Theorem 1.1 we put a remark explaining how to change, if necessary, the proof in order to obtain a proof of an analogous step of Theorem 5.1. In particular, we use similar notations for the projective and c-projective cases.

5.1. Conventions and degree of mobility. Within the whole $\S 5$ (except Remarks 1-9 for Theorem 5.1 where we use similar notation in the projective setting) we assume that $(M, g, J)$ is a closed connected Kähler manifold of (a priori) arbitrary signature and of dimension $2 n \geq 4$ (though the case $2 n=4$ has been settled in [10]), and that $v$ is a c-projective vector field which is not an affine vector field. We denote by $\Phi_{t}^{v}$ its flow, by definition the pullback of $g$ w.r.t. $\Phi_{v}^{t}$ is a metric which is c-projectively equivalent to $g$.

We define the degree of (c-projective) mobility $D(g, J)$ of $(g, J)$ as the dimension of the space of hermitian solutions of equation (1.4). If $D(g, J)=1$, the flow of $v$ acts by homotheties, since otherwise $\Phi_{t}^{*} g$ is non-proportional to $g$ and hence $D(g, J) \geq 2$. Thus, this case is impossible since we assumed that $v$ is not an affine vector field.

Now, in the case $D(g, J) \geq 3$, Theorem 1.1 follows from [19, Theorem 1], where it is proved that a closed connected Kähler manifold of arbitrary signature with $D(g, J) \geq 3$ is either $(\mathbb{C} P(n), c$. $g_{F S}, J_{\text {standard }}$ ) for some $c \in \mathbb{R} \backslash\{0\}$ or any metric which is c-projectively equivalent to $g$ is affinely equivalent to $g$. Thus, if $D(g, J) \geq 3$, we are done. In the remaining part of this section, we shall treat the case $D(g, J)=2$.

Here is our first remark related to the proof of Theorem 5.1.

Remark 1 for Theorem 5.1. For a (pseudo)-Riemannian metric $g$, the degree of (projective) mobility $D(g)$ is the dimension of the vector space of $g$-selfadjoint solutions of (1.7). If $D(g)=1$, every projective transformation is a homothety and is an affine transformation. If $D(g) \geq 3$ and $g$ has indefinite signature, then by [27, Corollary 5.2] (which plays here the role analogous to the role of [19] in the Kähler setting) each projective transformation is an affine transformation. Therefore, in the rest of the proof of Theorem 1.1 we may (and will) assume that $D(g)=2$.

5.2. Scheme of the proof. Let us outline the steps of the proof of Theorem 1.1 under the assumption $D(g, J)=2$. In $\S 5.3$, we will derive the PDE's that describe the evolution of the c-compatible pair $g$ and $A$ along a projective vector field $v$.

In $\S 5.4$ we show that the equation for $A$ can be reduced to one of three a priori possible canonical forms. By using these forms we show that $A$ cannot have non-constant complex eigenvalues and moreover, the real eigenvalues are bounded only for one particular canonical form, namely $\mathcal{L}_{v} A=A(\mathrm{Id}-A)$. This equation will automatically imply that the constant eigenvalues of $A$, if they exist, are 0 and 1.

This simplifies the formulas in Theorem 1.6 considerably. In the local classification, $g$ and $A$ are in block-diagonal form. In $\S 5.5$, we will obtain a partial solution to the PDE system by only considering the part that corresponds to the block spanned by the gradients of the nonconstant eigenvalues $\rho_{1}, \ldots, \rho_{\ell}$ of $A$ (that is the $L$-block from Theorem 1.5 and Example 3 ). The PDE system restricted to this block reduces to ordinary differential equations on a certain set of functions $F_{1}\left(\rho_{1}\right), \ldots, F_{\ell}\left(\rho_{\ell}\right)$ and on the eigenvalues $\rho_{i}$. Thus, we obtain quite explicit formulas for $A, g$ and $v$ involving some yet unknown constants $a_{1}, \ldots, a_{\ell}$ and $\mathcal{C}$ as parameters. 
In $§ 5.6$, we will use these formulas to analyse the asymptotic properties of the scalar products $g\left(K_{i}, K_{j}\right)$ of the Killing vector fields and the eigenvalues of the curvature operator. We will conclude from this analysis that there cannot be more than one non-constant eigenvalue $\rho$ (otherwise the eigenvalues of the curvature operator are unbounded for $t \rightarrow \pm \infty$ (which is impossible on a closed manifold) or $g\left(K_{i}, K_{j}\right)$ does not tend to zero for $t \rightarrow \pm \infty$ (as it should)).

Now we are left with a very specific form of the formulas in Theorem 1.6: there is only one non-constant eigenvalue $\rho$ and at most two constant eigenvalues 0 and 1 . In $\S 5.7$ we complete the proof of Theorem 1.1. We do this by deriving further restrictions on the constant $\mathcal{C}$ from above that appears as a parameter in the metric. Using results of [19] we are then able to conclude that the metric, up to a sign, is positive definite. This traces back the proof of Theorem 1.1 to the corresponding result [31] for positive signature.

5.3. C-projectively invariant form of equation (1.4) and special features of $D(g, J)=2$. Consider the canonical line bundle $\mathcal{E}=\Lambda^{2 n} T^{*} M$ over the Kähler manifold $(M, g, J)$. For a real number $w$, we define the line bundle $\mathcal{E}(w)$ whose transition functions are given by the transition functions of $\mathcal{E}$ to the power $w$. Note that $M$, as a complex manifold, has a canonical orientation and we can assume positivity of the transition functions of $\mathcal{E}$. For an arbitrary tensor bundle $E$ over $M$, we can then define the "weighted version" $E(w)=E \otimes \mathcal{E}(w)$. Let $S_{J}^{2} T M$ denote the bundle of hermitian contravariant 2-tensors and denote by $\nabla$ the Levi-Civita connection of $g$. In the Appendix of [31] it was shown that the PDE

$$
\nabla_{X} \sigma=X \odot \Lambda_{\sigma}+J X \odot J \Lambda_{\sigma}, \quad X \in T M
$$

on sections $\sigma$ of $S_{J}^{2} T M\left(\frac{1}{n+1}\right)\left(2 n=\operatorname{dim}_{\mathbb{R}} M\right)$, where $\Lambda_{\sigma} \in \Gamma\left(T M\left(\frac{1}{n+1}\right)\right)$ is the $\nabla$-divergence of $\sigma$ divided by $2 n$ and $X \odot Y=X \otimes Y+Y \otimes X$ denotes the symmetric product, is c-projectively invariant, that is, it does not depend on the choice of connection $\nabla$ in the class [ $\nabla]$ of c-projectively equivalent connections. Let us denote by $\mathcal{A}([g], J)$ the space of solutions of (5.1). There is an isomorphism between the space $\mathcal{A}(g, J)$ of hermitian solutions to (1.4) (i.e., the space of hermitian endomorphism c-compatible with $(g, J))$ and $\mathcal{A}([g], J)$ given by

$$
\varphi: \mathcal{A}([g], J) \longmapsto \mathcal{A}(g, J), \varphi(\sigma)=\sigma \sigma_{g}^{-1},
$$

where

$$
\sigma_{g}=g^{-1} \otimes \operatorname{vol}_{g}^{\frac{1}{n+1}}
$$

and $\mathrm{vol}_{g}$ is the volume form of $g$. As described in more detail in the appendix of [31], taking the Lie derivative $\mathcal{L}_{v} \sigma$ of a solution $\sigma$ to (5.1) w.r.t. the c-projective vector field $v$ yields again a solution to (5.1). Thus, we obtain a linear mapping

$$
\mathcal{L}_{v}: \mathcal{A}([g], J) \longmapsto \mathcal{A}([g], J) .
$$

Under the assumption $D(g, J)=2$, we can chose a basis $\sigma, \hat{\sigma}$ of $\mathcal{A}([g], J)$ and find the equations

$$
\begin{gathered}
\mathcal{L}_{v} \sigma=\alpha \sigma+\beta \hat{\sigma}, \\
\mathcal{L}_{v} \hat{\sigma}=\gamma \sigma+\delta \hat{\sigma}
\end{gathered}
$$

for certain real numbers $\alpha, \beta, \gamma, \delta$.

Using (5.2) we can easily derive the Lie derivatives of $A \in \mathcal{A}(g, J)$ and $g$ along $v$.

Proposition 5.2. Let $v$ be a c-projective vector field for $g$ and $A \in \mathcal{A}(g, J), A \neq c \cdot \mathrm{Id}$. Then we have the equations

$$
\mathcal{L}_{v} A=-\beta A^{2}+(\delta-\alpha) A+\gamma \mathrm{Id}
$$

and

$$
\mathcal{L}_{v} g=\left(-\frac{\beta}{2} \operatorname{tr} A-(n+1) \alpha\right) g-\beta g A,
$$

for some constants $\alpha, \beta, \gamma, \delta \in \mathbb{R}$. Moreover, the restriction $\rho(t)=\rho\left(\Phi_{t}^{v}(p)\right)$ of every eigenvalue $\rho$ of $A$ to an integral curve of $v$ satisfies the $O D E$

$$
\dot{\rho}=-\beta \rho^{2}+(\delta-\alpha) \rho+\gamma .
$$


Proof. Take $\sigma=\sigma_{g}$ and $\hat{\sigma}=\phi^{-1}(A)=A \sigma_{g}$ as a basis of $\mathcal{A}([g], J)$. Then by using (5.2), we get

$$
\begin{gathered}
\mathcal{L}_{v} A=\mathcal{L}_{v}\left(\hat{\sigma} \sigma_{g}^{-1}\right)=\left(\gamma \sigma_{g}+\delta \hat{\sigma}\right) \sigma_{g}^{-1}-\hat{\sigma} \sigma_{g}^{-1}\left(\alpha \sigma_{g}+\beta \hat{\sigma}\right) \sigma_{g}^{-1} \\
=\gamma \mathrm{Id}+\delta A-\alpha A-\beta A^{2} .
\end{gathered}
$$

This yields (5.3). Moreover, a straightforward calculation yields

$$
\begin{gathered}
\mathcal{L}_{v} g=\mathcal{L}_{v}\left((\operatorname{det} g)^{\frac{1}{2(n+1)}} \sigma_{g}^{-1}\right)=\frac{1}{2(n+1)} \operatorname{tr}\left(g^{-1} \mathcal{L}_{v} g\right) g-g\left(\alpha \sigma_{g}+\beta \sigma\right) \sigma_{g}^{-1} \\
=\left(\frac{1}{2(n+1)} \operatorname{tr}\left(g^{-1} \mathcal{L}_{v} g\right)-\alpha\right) g-\beta g A .
\end{gathered}
$$

Taking the trace of this equation gives us

$$
\frac{1}{2(n+1)} \operatorname{tr}\left(g^{-1} \mathcal{L}_{v} g\right)=-\alpha n-\frac{\beta}{2} \operatorname{tr} A
$$

and inserting this back into the equation for the Lie derivative of $g$, we obtain (5.4).

The remaining formula (5.5) immediately follows from (5.3).

Remark 2 for Theorem 5.1. The projective (and projectively invariant) analogue of (5.1) was obtained in [18]. Arguing as above we obtain the following version of Proposition 5.2:

Let $v$ be a projective vector field for $g$ and let $A$ be compatible to $g$ in the projective sense, $A \neq c \cdot$ Id. Then we have (5.3) and (5.5) and the Lie derivative of $g$ satisfies

$$
\mathcal{L}_{v} g=(-\beta \operatorname{tr} A-(n+1) \alpha) g-\beta g A, \quad n=\operatorname{dim} M,
$$

i.e., in the first term on the right-hand side of (5.4) we simply need to replace $\frac{\beta}{2}$ by $\beta$.

5.4. Properties of the eigenvalues of $A$. The equation (5.5) allows us to make several important conclusions about the eigenvalues of $A \in \mathcal{A}(g, J)$.

First of all we notice that the coefficient $\beta$ in equation (5.5) does not vanish. Indeed, otherwise this equation takes the form $\dot{\rho}=(\delta-\alpha) \rho+\gamma$ and its non-constant solutions are unbounded which is impossible due to compactness of $M$. Hence the eigenvalues of $A$ are all constant which contradicts the assumption that $v$ is not affine. Thus, $\beta \neq 0$.

Next, we see that the constant eigenvalues of $A$ are solutions to the quadratic equation $0=$ $-\beta x^{2}+(\delta-\alpha) x+\gamma$. Hence, there are at most two constant eigenvalues.

To simplify the further discussion, without loss of generality we may assume that the evolution of a non-constant eigenvalue $\rho$ of $A$ along $v$ is given by one of the ODEs

$$
\dot{\rho}=\rho^{2}+1, \quad \dot{\rho}=\rho(1-\rho) \text { or } \dot{\rho}=\rho^{2} .
$$

Indeed, the equations (5.5) can be reduced to one of these canonical forms by rescaling $v$ and replacing $A$ by $c_{1} A+c_{2}$ Id for an appropriate choice of constants $c_{1} \neq 0, c_{2} \in \mathbb{R}$.

We now show that the eigenvalues of $A$ cannot be complex.

Proposition 5.3. Let $(M, g, J)$ be a closed connected Kähler manifold of degree of mobility $D(g, J)=2$ and let $v$ be a c-projective vector field which is not affine. Then all non-constant eigenvalues of $A \in \mathcal{A}(g, J)$ are real-valued.

Proof. If we allow $\rho$ to be complex-valued, each of the above ODEs (5.6) should be considered as a system of two ODEs on the real and imaginary part of $\rho$. The phase portraits corresponding to these systems are shown in Figure 1. It can be seen from the pictures or shown directly using the ODEs (5.6), that a non-constant solution to one of these equations with imaginary part not identically zero is given by a circle in the complex plane.

As we see from Figure 1, the maximal value of an imaginary part of a complex eigenvalue, taken over all points of the manifold and all eigenvalues, is not equal to the imaginary part of a constant eigenvalue (for each case in (5.6), the constant eigenvalues are $\pm i, 0$ and 1 or 0 resp.). In particular, the derivative of this eigenvalue in the direction of the c-projective vector field is well-defined and non-zero. More precisely, the derivative of the imaginary part of $\rho$ is zero whereas the derivative of the real part is not. We now show that for complex eigenvalues such a situation is impossible. 

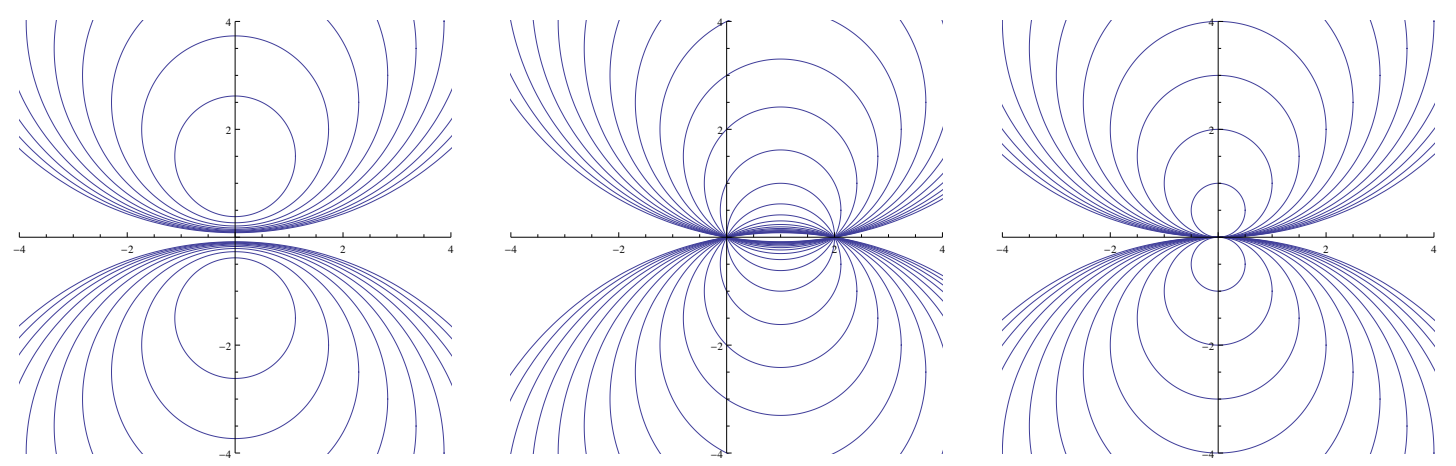

Figure 1. The phase portraits for the ODEs $\dot{\rho}=\rho^{2}+1, \dot{\rho}=\rho(1-\rho), \dot{\rho}=\rho^{2}$ (from left to right).

Basically this fact follows from our local description of c-projectively equivalent metrics (Theorem 1.6) which states that the complex eigenvalues are holomorphic in an appropriate local coordinate system. However, such a coordinate system exists only at generic points, so we need to modify this idea and in particular to take into account the fact that the eigenvalues cannot be considered as smooth functions at "collision" points where the multiplicities of the eigenvalues change.

Assume that at a point $p \in M$, a complex eigenvalue $\rho$ of $A, \operatorname{Im} \rho(p) \neq 0$, has multiplicity $k$. It follows from standard facts that for small neighbourhoods $U(p) \subseteq M$ of $p$ and $U(\rho(p)) \subseteq \mathbb{C}$ of $\rho(p), A$ has precisely $k$ eigenvalues $\rho_{1}, \ldots, \rho_{k}$ at each point of $U(p)$ contained in $U(\rho(p))$ and that the elementary symmetric functions in the variables $\rho_{1}, \ldots, \rho_{k}$ are smooth complex-valued functions on $U(p)$. In particular, the function $\rho_{1}+\cdots+\rho_{k}$ is smooth in $U(p)$.

Lemma 5.4. Let the differential of the imaginary part of $\rho_{1}+\cdots+\rho_{k}$ vanishes at $p$. Then

$$
\mathrm{d}\left(\rho_{1}+\cdots+\rho_{k}\right)=0 .
$$

Proof. By contradiction, assume that $\mathrm{d}\left(\rho_{1}+\cdots+\rho_{k}\right) \neq 0$. Consider the smooth endomorphism

$$
\tilde{A}=\left(A-\rho_{1} \cdot \mathrm{Id}\right) \ldots\left(A-\rho_{k} \cdot \mathrm{Id}\right): T_{\mathbb{C}}^{*} U(p) \rightarrow T_{\mathbb{C}}^{*} U(p),
$$

where $T_{\mathbb{C}}^{*} U(p)$ denotes the complexified cotangent bundle of $U(p)$. The kernel of $\tilde{A}$ defines a smooth complex $k$-dimensional distribution $D$ in $T_{\mathbb{C}}^{*} U(p)$ whose value $D(p)$ at the point $p$ coincides with the kernel of $(A-\rho(p) \cdot \mathrm{Id})^{k}$. The subspace $D(p)$ is therefore the generalised $\rho(p)$-eigenspace of $A$.

The differential of the smooth function $\rho_{1}+\cdots+\rho_{k}$ at a generic point of $U(p)$ is $\mathrm{d} \rho_{1}+\cdots+\mathrm{d} \rho_{k}$ and since $\mathrm{d} \rho_{i} \circ A=\rho_{i} \mathrm{~d} \rho_{i}$ (because, by Lemma 2.2, grad $\rho$ is an eigenvector with eigenvalue $\rho$ of the $g$-selfadjoint endomorphism $A)$, we obtain that $\mathrm{d}\left(\rho_{1}+\cdots+\rho_{k}\right)$ is contained in $D$ at every point of $U(p)$. In particular we have that at $p, \mathrm{~d}\left(\rho_{1}+\cdots+\rho_{k}\right)$ is contained in $D(p)$, i.e., it is a generalised eigenvector of $A$ corresponding to $\rho(p)$. On the other hand, since $\mathrm{d}\left(\operatorname{Im}\left(\rho_{1}+\cdots+\rho_{k}\right)\right)(p)=0$, $\mathrm{d}\left(\rho_{1}+\cdots+\rho_{k}\right)$ is a real 1 -form at $p$. This contradicts to the following simple fact from Linear Algebra: if $\rho$ is a complex eigenvalue of a real linear endomorphism $A$, then the generalised $\rho(p)$-eigenspace of $A$ contains no real vectors.

Now take a point $p \in M$ such that the imaginary part of an eigenvalue $\rho$ takes its maximum in the sense that it is greater or equal than the imaginary parts of all eigenvalues at all points of $M$ and apply Lemma 5.4. The imaginary part of $\rho_{1}+\cdots+\rho_{k}$ obviously satisfies $\mathrm{d}\left(\operatorname{Im} \sum \rho_{i}(p)\right)=0$. On the other hand, the Lie derivative of $\sum \rho_{i}$ along $v$ at the point $p$ is $k \cdot \dot{\rho} \neq 0$, where $\dot{\rho}$ is defined by one of the equations (5.6). Thus, $\mathrm{d}\left(\rho_{1}+\cdots+\rho_{k}\right) \neq 0$ in contradiction with Lemma 5.4. This completes the proof.

In fact, only the second equation of (5.6) is allowed. Indeed, since $v$ is not affine, $A$ must have at least one non-constant eigenvalue and, moreover, this eigenvalue has to be real according to Proposition 5.3. But it is easy to see that the equations $\dot{\rho}=\rho^{2}+1$ and $\dot{\rho}=\rho^{2}$ have no bounded real-valued non-constant solutions whereas $\rho(t)$ must be bounded due to compactness of $M$. 
Thus, we are left with the case when the eigenvalues of $A$ satisfy

$$
v(\rho)=\rho(1-\rho) .
$$

and we may summarize the above discussion in the following

Proposition 5.5. Let $(M, g, J)$ be a closed connected Kähler manifold of real dimension $2 n \geq 4$ such that $D(g, J)=2$ and let $v$ be a c-projective vector field that is not affine. Then, after an appropriate rescaling of $v$, we can find $A \in \mathcal{A}(g, J)$ such that

(1) the eigenvalues of $A$ satisfy (5.7);

(2) the eigenvalues of $A$ are all real;

(3) A has at most two constant eigenvalues 0 and 1.

Remark 3 for Theorem 5.1. The reduction of (5.5) to one of the canonical forms (5.6) is a simple general fact from the theory of ODE's. The statement of Proposition 5.3 was proved in [9, Theorem 1.11] even under more general assumptions: on a compact manifold $M$, each non-real eigenvalue of an endomorphism $A$ compatible with $g$ is necessarily constant.

The first and third canonical forms from (5.6) are impossible for the same reason: in these two cases non-constant real eigenvalues are not bounded (along $v$ ). Thus, the eigenvalues of $A$ satisfy (5.7) and the statement of Proposition 5.5 remains true without any changes.

5.5. Explicit formulas for the non-constant block. In what follows, we will work with $A \in$ $\mathcal{A}(g, J)$ and a c-projective vector field $v$ as in Proposition 5.5. In particular, we assume that the non-constant eigenvalues $\rho_{1}, \ldots, \rho_{\ell}$ of $A$ are real-valued and the constant eigenvalues, 0 and 1 , have multiplicities $m_{0} \geq 0$ and $m_{1} \geq 0$ respectively.

Under these assumptions, the PDE systems (5.3) and (5.4) take the form

$$
\begin{gathered}
\mathcal{L}_{v} A=A(\mathrm{Id}-A), \\
\mathcal{L}_{v} g=-g A-\left(\sum_{i=1}^{\ell} \rho_{i}+\mathcal{C}\right) g .
\end{gathered}
$$

where $\mathcal{C}$ is some constant (yet unknown and playing the role of an additional parameter).

We are going to evaluate the PDE system (5.8) component-wise. To make this more transparent, we notice that on the set of regular points $M^{0}$ there is a natural structure of two mutually orthogonal foliations. Recall that $\mathcal{F}$ denotes the integrable and totally geodesic distribution spanned by the commuting vector fields given by the Killing vector fields $K_{1}, \ldots, K_{\ell}$ and the vector fields $J K_{1}, \ldots, J K_{\ell}$. Now let $\mathcal{U}$ be the distribution generated by $\operatorname{grad} \rho_{1}, \ldots, \operatorname{grad} \rho_{\ell}$ (or, equivalently, by $\left.J K_{1}, \ldots, J K_{\ell}\right)$ so that $\mathcal{F}=\mathcal{U} \oplus \mathcal{V}$, where $\mathcal{V}$ is the distribution generated by $K_{1}, \ldots, K_{\ell}$ and defined in the first part of the article. The leaves of the other distribution $\mathcal{U}^{\perp}$, orthogonal to $\mathcal{U}$, are just common level surfaces of the eigenvalues $\rho_{1}, \ldots, \rho_{\ell}$. Note that both distributions are integrable: for $\mathcal{U}^{\perp}$ this holds by definition and for $\mathcal{U}$ it follows from the fact that $\mathcal{U}$ is generated by the commuting vector fields $J K_{1}, \ldots, J K_{\ell}$.

The next statement summarizes some general properties of $\mathcal{U}$ which hold true for any metric $g$ from Theorem 1.6.

Proposition 5.6. Let $M^{0} \subset M$ be the set of regular points (in particular, the non-constant eigenvalues $\rho_{1}, \ldots, \rho_{\ell}$ of $A$ are all distinct and independent on $\left.M^{0}\right)$. Then on $M^{0}$ there is a structure of the integrable distribution $\mathcal{U}$ generated by $\operatorname{grad} \rho_{i}(i=1, \ldots, \ell)$ with the following properties:

(1) The leaves of $\mathcal{U}$ are totally geodesic.

(2) The leaves of $\mathcal{U}^{\perp}$ are common level surfaces of $\rho_{1}, \ldots, \rho_{\ell}$.

(3) Let $\mathcal{L} \subset M^{0}$ be a leaf of $\mathcal{U}$, then $\left.g\right|_{\mathcal{L}}$ and $\left.A\right|_{\mathcal{L}}$ are compatible in the projective sense.

(4) The non-constant eigenvalues $\rho_{1}, \ldots, \rho_{\ell}$ can be considered as local coordinates on $\mathcal{L}$.

(5) Locally the metric $g$ can be written as

$$
g=\sum_{i=1}^{\ell} g_{i}(\vec{\rho}) \mathrm{d} \rho_{i}^{2}+\sum_{\alpha, \beta} b_{\alpha \beta}(\rho, y) \mathrm{d} y_{\alpha} \mathrm{d} y_{\beta},
$$

where $\sum_{i=1}^{\ell} g_{i}(\vec{\rho}) \mathrm{d} \rho_{i}^{2}$ is $\left.g\right|_{\mathcal{L}}$. 
(6) The vector fields $\operatorname{grad} \rho_{i}$ on $M$ and on $\mathcal{L}$ coincide (no matter which metric, $g$ or $\left.g\right|_{\mathcal{L}}$, is used to take the gradient) and therefore the quantities $g\left(\operatorname{grad} f_{1}(\vec{\rho}) \operatorname{grad} f_{2}(\vec{\rho})\right)$ do not depend on how we compute them (on $M$ or on $\mathcal{L}$ ).

(7) The vector field $\Lambda=\frac{1}{4} \operatorname{grad}(\operatorname{tr} A)$ is the same on $\mathcal{L}$ and $M$. The tangent space of $\mathcal{L}$ is invariant with respect to the endomorphism $\nabla \Lambda$, moreover, the restriction of $\nabla \Lambda$ on $\mathcal{L}$ coincides with $\left.\nabla\right|_{\mathcal{L}} \Lambda$ computed on $\mathcal{L}$. This implies in particular that the eigenvalues of $\left.\nabla\right|_{\mathcal{L}} \Lambda$ are some of the eigenvalues of $\nabla \Lambda$.

(8) The leaves of $\mathcal{U}$ are (locally) isometric.

Proof. In view of $\nabla J=0$ and the fact that $K_{i}, J K_{i}$ are holomorphic, (1) follows immediately from Lemma $2.2(8)$.

(2) is clear from the definition.

(3) follows immediately from (1) combined with equation (1.4) (compare also Lemma 3.3).

(4) follows from the assumption that $\rho_{1}, \ldots, \rho_{\ell}$ are independent.

(5) is immediate from the local classification Theorem 1.6 and (6) follows directly from (5). The first statement of (7) follows from (6) and the formula $\Lambda=\frac{1}{2} \sum_{i=1}^{\ell} \operatorname{grad} \rho_{i}$. The remaining statements of (7) then follow directly from (1).

The last statement (8) can be seen from the formula for $g$ in (5).

Remark 4 for Theorem 5.1. In the projective setting, the definition of the set $M^{0}$ of regular points remains essentially the same. We say that $p \in M$ is regular if: 1) the algebraic type of $A$ does not change in a (sufficiently small) neighbourhood $U$ of $p, 2$ ) each eigenvalue $\rho$ is either constant on $U$ (and then $\rho$ equals either 0 or 1 ) or, if $\rho$ is not constant on $U$, then $\mathrm{d} \rho(p) \neq 0$. Recall that in view of (5.7):

$$
\rho(p) \neq 0 \text { or } 1 \Rightarrow \mathrm{d} \rho(p) \neq 0 .
$$

Clearly, $M^{0}$ is open and everywhere dense on $M$. However, a priori $M^{0}$ might contain several components related to different algebraic types. In particular, the number $\ell$ of non-constant eigenvalues may be different for different components of $M^{0}$. We continue to work with one of the components of $M^{0}$ (for simplicity, we will still denote this component by $M^{0}$ ). Since we want to prove Theorem 5.1 by contradiction, we assume from now on that there is a regular point (or, which is the same, one of the connected components of $M^{0}$ ) where the non-constant part of $A$ is diagonalisable (over $\mathbb{R}$ ), i.e. with no Jordan blocks. Recall from [9] that in this case each non-constant eigenvalue has multiplicity one.

Summarizing we have an open subset $M^{0} \subset M$ with the following properties:

(1) at each point $p \in M^{0}$, the endomorphism $A$ has $\ell$ non-constant real eigenvalues $\rho_{1}<\cdots<$ $\rho_{\ell}$, each of multiplicity one;

(2) $\mathrm{d} \rho_{i} \neq 0$ everywhere on $M^{0}$;

(3) the "constant part" of $A$ has some fixed algebraic type with at most two eigenvalues 0 and 1 of multiplicity $m_{0}$ and $m_{1}$ respectively.

In particular, according to [9], locally in a neighbourhood of every point $p \in M^{0}$, we can choose a coordinate system $\rho_{1}, \ldots, \rho_{\ell}, y_{1}, \ldots, y_{s}$ (cf. Proposition 4.1) in which both $g$ and $A$ split:

$$
g=\left(\begin{array}{cc}
h(\rho) & 0 \\
0 & g_{\mathrm{c}}(y) \cdot \chi_{L}\left(A_{\mathrm{c}}(y)\right)
\end{array}\right) \text { and } A=\left(\begin{array}{cc}
L(\rho) & 0 \\
0 & A_{\mathrm{c}}(y)
\end{array}\right)
$$

Here $L=\operatorname{diag}\left(\rho_{1}, \ldots, \rho_{\ell}\right)$, the metric $h$ and the endomorphism $L$ are compatible in the projective sense, $A_{\mathrm{c}}$ is parallel w.r.t. $g_{\mathrm{c}}$ and $\chi_{L}(t)=\left(t-\rho_{1}\right)\left(t-\rho_{2}\right) \ldots\left(t-\rho_{\ell}\right)$ is the characteristic polynomial of $L$. This decomposition into "constant" and "non-constant" blocks can naturally be reformulated in terms of two orthogonal foliations $\mathcal{U}$ and $\mathcal{U}^{\perp}$. Proposition 5.6 remains true without any changes.

Also notice that (5.8) holds without any change in spite of the fact that in (5.4) we should replace $\frac{\beta}{2}$ by $\beta$. This happens because $\frac{1}{2}$ is compensated by the factor 2 in the formula for the trace in the c-projective setting where $\operatorname{tr} A=2 \sum \rho_{i}+$ const (whereas in the real case $\operatorname{tr} A=\sum \rho_{i}+$ const).

The further analysis deals with the restrictions of $g$ and $v$ to leaves of $\mathcal{U}$. These restrictions are exactly the same for the projective and c-projective cases and, until §5.7, the proof for the both cases will be the same. The difference which appears in $\S 5.7$ will be clearly described. 
We now want to derive explicit formulas for the restrictions of $g$ and $v$ to leaves of $\mathcal{U}$. This is sufficient for many discussions since some of the globally defined objects derived from $g$ and $A$ only depend on the level sets of the non-constant eigenvalues, see $\S 5.6$ below.

We first notice that according to Theorem 1.6, at regular points $p \in M^{0}$ we have $\rho_{i}=\rho_{i}\left(x_{i}\right)$ and moreover $\frac{\partial \rho_{i}}{\partial x_{i}} \neq 0$. This means, in particular, that we can use $\rho_{i}$ as local coordinates instead of $x_{i}$. This change of variables will simplify further computations.

Let $v=v_{1}+v_{2}$ be the decomposition of the c-projective vector field $v$ w.r.t. $T M=\mathcal{U} \oplus \mathcal{U}^{\perp}$. According to Proposition 5.6, there are certain functions $v_{1}^{i}$ such that $v$ can be written as

$$
v=\sum_{i=1}^{\ell} v_{1}^{i} \frac{\partial}{\partial \rho_{i}}+v_{2} .
$$

Since each eigenvalue $\rho_{i}$ satisfies (5.7) and is constant in the direction of $\mathcal{U}^{\perp}$, we immediately see that

$$
v\left(\rho_{i}\right)=v_{1}^{i} \frac{\partial \rho_{i}}{\partial \rho_{i}}=\rho_{i}\left(1-\rho_{i}\right),
$$

that is, $v_{1}^{i}=\rho_{i}\left(1-\rho_{i}\right)$.

From Theorem 1.6, we know that in our simplified situation (no complex non-constant eigenvalues), $g$ and $g A$ can be written in the form (after the above change of variables $x_{i} \leftrightarrow \rho_{i}$ )

$$
\begin{gathered}
g=\sum_{i=1}^{\ell} \frac{\Delta_{i}}{F_{i}} \mathrm{~d} \rho_{i}^{2}+\sum_{i, j=1}^{\ell} H_{i j} \theta_{i} \theta_{j}+g_{\mathrm{c}}\left(\chi_{\mathrm{nc}}\left(A_{\mathrm{c}}\right) \cdot, \cdot\right), \\
g A=\sum_{i=1}^{\ell} \rho_{i} \frac{\Delta_{i}}{F_{i}} \mathrm{~d} \rho_{i}^{2}+\sum_{i, j=1}^{\ell} \tilde{H}_{i j} \theta_{i} \theta_{j}+g_{\mathrm{c}}\left(\chi_{\mathrm{nc}}\left(A_{\mathrm{c}}\right) A_{\mathrm{c}} \cdot, \cdot\right),
\end{gathered}
$$

where $\Delta_{i}=\prod_{j \neq i}\left(\rho_{i}-\rho_{j}\right), F_{i}=\varepsilon_{i}\left(\frac{\partial \rho_{i}}{\partial x_{i}}\right)^{2}$, the functions $H_{i j}, \tilde{H}_{i j}$ only depend on $\rho_{1}, \ldots, \rho_{\ell}$ and $\chi_{\mathrm{nc}}(t)=\prod_{i=1}^{\ell}\left(t-\rho_{i}\right)$.

Proposition 5.7. Locally, the metric $g$ and the c-projective vector field $v$ in the coordinates $\rho_{1}, \ldots, \rho_{\ell}$ are given by the formulas

$$
g=\sum_{i=1}^{\ell} \frac{\Delta_{i}}{F_{i}} \mathrm{~d} \rho_{i}^{2}+\ldots, \quad v=\sum_{i=1}^{\ell} \rho_{i}\left(1-\rho_{i}\right) \frac{\partial}{\partial \rho_{i}}+\ldots,
$$

where $\Delta_{i}=\prod_{j \neq i}\left(\rho_{i}-\rho_{j}\right)$ and

$$
F_{i}(t)=a_{i}(1-t)^{-\mathcal{C}} t^{1+\ell+\mathcal{C}} .
$$

for some real constants $a_{i}$ and $\mathcal{C}$.

Note that the term $g-\sum_{i=1}^{\ell} \frac{\Delta_{i}}{F_{i}} \mathrm{~d} \rho_{i}^{2}$ in formula (5.11), which is not written down explicitly, coincides with $\sum_{\alpha, \beta} b_{i j}(\rho, y) d y_{\alpha} d y_{\beta}$ from part (5) of Proposition 5.6. The expression $v-\sum_{i=1}^{\ell} \rho_{i}(1-$ $\left.\rho_{i}\right) \frac{\partial}{\partial \rho_{i}}$ is just the projection of $v$ onto $\mathcal{U}^{\perp}$.

Proof. It easily follows from the explicit form of $g$ given by (5.10) that the second equation of (5.8) implies

$$
\mathcal{L}_{v_{1}}\left(\sum_{i=1}^{\ell} \frac{\Delta_{i}}{F_{i}} \mathrm{~d} \rho_{i}^{2}\right)=-\sum_{i=1}^{\ell}\left(\rho_{i}+\sum_{j=1}^{\ell} \rho_{j}+\mathcal{C}\right) \frac{\Delta_{i}}{F_{i}} \mathrm{~d} \rho_{i}^{2}
$$

where $v_{1}=\sum \rho_{j}\left(1-\rho_{j}\right) \frac{\partial}{\partial \rho_{j}}$. In other words, the first part of $g$ can be differentiated along $v$ independently of the remaining terms.

From this equation we can easily derive the explicit formulas for $F_{i}$. To that end, we compute the left hand side of (5.13): 


$$
\begin{aligned}
& \mathcal{L}_{v_{1}}\left(\sum_{i=1}^{\ell} \frac{\Delta_{i}}{F_{i}} \mathrm{~d} \rho_{i}^{2}\right)= \\
& \sum_{i=1}^{\ell} \sum_{j \neq i} \rho_{j}\left(1-\rho_{j}\right) \frac{\partial}{\partial \rho_{j}}\left(\frac{\Delta_{i}}{F_{i}}\right) \mathrm{d} \rho_{i}^{2}+\sum_{i=1}^{\ell} \rho_{i}\left(1-\rho_{i}\right) \frac{\partial}{\partial \rho_{i}}\left(\frac{\Delta_{i}}{F_{i}}\right) \mathrm{d} \rho_{i}^{2}+\sum_{i=1}^{\ell} \frac{\Delta_{i}}{F_{i}} L_{v_{1}}\left(\mathrm{~d} \rho_{i}^{2}\right)= \\
& \sum_{i=1}^{\ell} \sum_{j \neq i} \frac{\rho_{j}\left(1-\rho_{j}\right)}{\rho_{j}-\rho_{i}} \cdot \frac{\Delta_{i}}{F_{i}} \mathrm{~d} \rho_{i}^{2}+\sum_{i=1}^{\ell} \sum_{j \neq i} \frac{\rho_{i}\left(1-\rho_{i}\right)}{\rho_{i}-\rho_{j}} \cdot \frac{\Delta_{i}}{F_{i}} \mathrm{~d} \rho_{i}^{2}-\sum_{i=1}^{\ell} \rho_{i}\left(1-\rho_{i}\right) \frac{\partial F_{i}}{\partial \rho_{i}} \frac{\Delta_{i}}{F_{i}^{2}} \mathrm{~d} \rho_{i}^{2}+ \\
& +2 \sum_{i=1}^{\ell} \frac{\Delta_{i}}{F_{i}} \frac{\partial}{\partial \rho_{i}}\left(\rho_{i}\left(1-\rho_{i}\right)\right) \mathrm{d} \rho_{i}^{2}= \\
& \sum_{i=1}^{\ell} \sum_{j \neq i}\left(\frac{\rho_{j}\left(1-\rho_{j}\right)}{\rho_{j}-\rho_{i}}+\frac{\rho_{i}\left(1-\rho_{i}\right)}{\rho_{i}-\rho_{j}}\right) \cdot \frac{\Delta_{i}}{F_{i}} \mathrm{~d} \rho_{i}^{2}-\sum_{i=1}^{\ell} \rho_{i}\left(1-\rho_{i}\right) \frac{\partial F_{i}}{\partial \rho_{i}} \frac{\Delta_{i}}{F_{i}^{2}} \mathrm{~d} \rho_{i}^{2}+2 \sum_{i=1}^{\ell} \frac{\Delta_{i}}{F_{i}}\left(1-2 \rho_{i}\right) \mathrm{d} \rho_{i}^{2}= \\
& \sum_{i=1}^{\ell}\left(\sum_{j \neq i}\left(1-\rho_{i}-\rho_{j}\right)-\rho_{i}\left(1-\rho_{i}\right) \frac{\partial \ln F_{i}}{\partial \rho_{i}}+2\left(1-2 \rho_{i}\right)\right) \frac{\Delta_{i}}{F_{i}} \mathrm{~d} \rho_{i}^{2}= \\
& \sum_{i=1}^{\ell}\left(\ell-1-(\ell-2) \rho_{i}-\sum_{j=1}^{\ell} \rho_{j}-\rho_{i}\left(1-\rho_{i}\right) \frac{\partial \ln F_{i}}{\partial \rho_{i}}+2\left(1-2 \rho_{i}\right)\right) \frac{\Delta_{i}}{F_{i}} \mathrm{~d} \rho_{i}^{2}= \\
& \sum_{i=1}^{\ell}\left(\ell+1-(\ell+2) \rho_{i}-\sum_{j=1}^{\ell} \rho_{j}-\rho_{i}\left(1-\rho_{i}\right) \frac{\partial \ln F_{i}}{\partial \rho_{i}}\right) \frac{\Delta_{i}}{F_{i}} \mathrm{~d} \rho_{i}^{2}
\end{aligned}
$$

Comparing this expression with the right hand side of (5.13), we obtain a simple ODE on $F_{i}$ :

$$
\ell+1-(\ell+2) \rho_{i}-\sum_{j=1}^{\ell} \rho_{j}-\rho_{i}\left(1-\rho_{i}\right) \frac{\partial \ln F_{i}}{\partial \rho_{i}}=-\left(\rho_{i}+\sum_{j=1}^{\ell} \rho_{j}+\mathcal{C}\right)
$$

which after simplification becomes

$$
\frac{\partial \ln F_{i}}{\partial \rho_{i}}=\frac{\ell+1+\mathcal{C}-(\ell+1) \rho_{i}}{\rho_{i}\left(1-\rho_{i}\right)}=\frac{\mathcal{C}}{1-\rho_{i}}+\frac{1+\ell+\mathcal{C}}{\rho_{i}}
$$

and we get

$$
F_{i}=a_{i}\left(1-\rho_{i}\right)^{-\mathcal{C}} \rho_{i}^{1+\ell+\mathcal{C}},
$$

as required.

In the proof of Proposition 5.7, we have actually shown that for $D(g, J)=2$, not only $g$ and $A$ restricted to integral leaves $\mathcal{L}$ of $\mathcal{U}$ are compatible in the projective sense, but also the projection $v_{1}$ of $v$ to $\mathcal{L}$ is a projective vector field for $h=\left.g\right|_{\mathcal{L}}$. Indeed, the second equation of (5.8) we used in the construction implies the second equation of (5.14) which is equivalent to the property of $v_{1}$ to be a projective vector field for $h$. Moreover, we already know that $v_{1}=v_{1}(\rho)$ depends only on $\rho$ which is equivalent to the fact that $v$ preserves the distribution $\mathcal{U}^{\perp}$. Similarly, we have that $v_{2}=v_{2}(y)$ (in the notation of Proposition 5.6) for the component of $v$ tangent to $\mathcal{U}^{\perp}$ or, equivalently, that $v$ preserves $\mathcal{U}$. To see this, note that we have $\left[v, K_{i}\right]\left(\rho_{j}\right)=0$ for all $i, j=1, \ldots, \ell$, hence, $\left[v, K_{i}\right] \in \mathcal{U}^{\perp}$. Since $v$ is holomorphic and, by the first equation in (5.8), preserves the generalised eigenspaces of $A$, we obtain that $\left[v, J K_{i}\right] \in \mathcal{U}$ for all $i=1, \ldots, \ell$, hence, $v$ preserves $\mathcal{U}$. We summarize this discussion in the following

Corollary 5.8. Let $v$ be a c-projective vector field and $D(g, J)=2$. Consider the natural decomposition of $v$ associated with the distributions $\mathcal{U}$ and $\mathcal{U}^{\perp}$ :

$$
v=v_{1}+v_{2}, \quad v_{1} \in \mathcal{U}, v_{2} \in \mathcal{U}^{\perp} .
$$


Let $\mathcal{L}$ denote an integral leaf of $\mathcal{U}$ and denote by $h=\left.g\right|_{\mathcal{L}}$ and $L=\left.A\right|_{\mathcal{L}}$ the restrictions of $g$ resp. $A$ to $\mathcal{L}$. Then

(1) The vector field $v$ preserves the both distributions $\mathcal{U}$ and $\mathcal{U}^{\perp}$, that is, $v_{1}=v_{1}(\rho)$ and $v_{2}=v_{2}(y)$ (in the notation of Proposition 5.6).

(2) The projection $v_{1}$ of $v$ onto $\mathcal{L}$ is a projective vector field for $h$ and the equations (5.8) can be naturally restricted onto $\mathcal{L}$, namely we have

$$
\begin{aligned}
& \mathcal{L}_{v_{1}} L=L(\mathrm{Id}-L), \\
& \mathcal{L}_{v_{1}} h=-h L-\left(\sum_{i=1}^{\ell} \rho_{i}+\mathcal{C}\right) h .
\end{aligned}
$$

5.6. There is only one non-constant eigenvalue. The goal of this subsection is to prove

Proposition 5.9. Let $A \in \mathcal{A}(g, J)$ and the assumptions be as in Proposition 5.5. Then $A$ cannot have more than one non-constant eigenvalue.

The idea of the proof is based on the analysis of geometric properties of the metric $g$ given by (5.11) or, more precisely, of the restriction $h=\left.g\right|_{\mathcal{L}}$. By construction these explicit formulas for $g$ are local, but we will show that they make sense for all admissible values of $\rho_{i}$.

Proposition 5.10. Consider the domain $U=\left\{0<\rho_{1}<\cdots<\rho_{\ell}<1\right\}$ with the metric

$$
\sum_{i=1}^{\ell} \frac{\Delta_{i}}{F_{i}} \mathrm{~d} \rho_{i}^{2}
$$

(compare (5.11)). There is a natural isometric embedding of $\phi: U \rightarrow M$ (as a maximal leaf of the totally geodesic foliation $\mathcal{U})$.

Proof. Locally, $\phi$ is defined in a very natural way. We choose a particular leaf $\mathcal{L}$ with $\rho_{i}$ as local coordinates and say that $\phi\left(\rho_{1}, \ldots, \rho_{\ell}\right)$ is the point on $\mathcal{L}$ with the same coordinates $\rho_{1}, \ldots, \rho_{\ell}$. We start with a certain point $a_{0} \in U$ and then extend this map as long as we can. The map $\phi$ so obtained is obviously an isometry. We need to show that such a prolongation can be made to any point of $U$.

The argument is standard. Consider a smooth curve $a(t)$ with $a(0)=a_{0} \in U$ and $a(1)=a_{1} \in U$, and choose $T_{0} \in[0,1]$ to be the supremum of those $T \in[0,1]$ for which the extension along the curve $a(t), t \in[0, T]$, is well defined. Take the image $\phi(a(t)), t \in\left[0, T_{0}\right)$. Since $M$ is compact, we can find a limit point $p$ of $\phi(a(t))$ as $t \rightarrow T_{0}$. By continuity, the eigenvalues of $A(p)$ coincide with the coordinates of $a\left(T_{0}\right)$ in $U$, i.e. $0<\rho_{1}<\cdots<\rho_{\ell}<1$. But this condition guarantees that $p \in M^{0}$, i.e. in a neighbourhood of $p$, the foliation $\mathcal{U}$ is defined. This obviously implies that $p \in \mathcal{L}$, moreover $p=\phi\left(a\left(T_{0}\right)\right)$ and we can extend $\phi$ to some neighbourhood of $a\left(T_{0}\right) \in U$. In particular, $T_{0}$ cannot be an interior point of $[0,1]$. In other words, the prolongation of $\phi$ along $a(t)$ is well defined for all $t \in[0,1]$.

Consider the function $f: U \rightarrow \mathbb{R}:$

$$
f(\vec{\rho})=h\left(\operatorname{grad} \sum_{i=1}^{\ell} \rho_{i}, \operatorname{grad} \sum_{i=1}^{\ell} \rho_{i}\right),
$$

where $h$ is the metric on $U$ defined explicitly by (the first term of $g$ in) (5.11).

According to Proposition 5.10, we can naturally identify the domain $U=\left\{0<\rho_{1}<\cdots<\rho_{\ell}<\right.$ $1\}$ with a leaf $\mathcal{L}$ of $\mathcal{U}$. Moreover, the function $f$ can be considered (up to a constant multiple) as the restriction to $\mathcal{L}$ of the function $g(\operatorname{grad} \operatorname{tr} A$, grad $\operatorname{tr} A)$ which is globally defined and smooth on $M$ (see part (6) of Proposition 5.6). This immediately implies certain conditions on $f(\vec{\rho})$.

Proposition 5.11. The function $f(\vec{\rho})$ must be bounded on $U$. Moreover, we have $f(\vec{\rho}) \rightarrow 0$ as $\left(\rho_{1}, \ldots, \rho_{\ell}\right) \rightarrow(0, \ldots, 0)$ or $\left(\rho_{1}, \ldots, \rho_{\ell}\right) \rightarrow(1, \ldots, 1)$.

Proof. The first claim follows from the compactness of $M$. Since $\operatorname{tr} A$ takes its minimum resp. maximum value at the limit points $(0, \ldots, 0)$ resp. $(1, \ldots, 1)$, we obtain that $\operatorname{grad} \operatorname{tr} A$ tends to zero if $\left(\rho_{1}, \ldots, \rho_{\ell}\right)$ tends to $(0, \ldots, 0)$ or $(1, \ldots, 1)$. The proves the proposition. 
These conditions will give us some further restrictions on $g$. It is straightforward to compute the function $f(\vec{\rho})$ explicitly using $(5.11)$ :

$$
f(\vec{\rho})=\sum_{i=1}^{\ell} \frac{F_{i}}{\Delta_{i}}=\sum_{i=1}^{\ell} \frac{a_{i}\left(1-\rho_{i}\right)^{-\mathcal{C}} \rho_{i}^{1+\ell+\mathcal{C}}}{\prod_{j \neq i}\left(\rho_{i}-\rho_{j}\right)}
$$

To study the limiting behaviour of such functions we use the following

Lemma 5.12. (1) Let $k_{1}, \ldots, k_{\ell}$ be functions of one variable. Then the function

$$
f=\sum_{i=1}^{\ell} \frac{k_{i}\left(\rho_{i}\right)}{\prod_{j \neq i}\left(\rho_{i}-\rho_{j}\right)},
$$

defined on the domain $\rho_{1}<\cdots<\rho_{\ell}$, is equal to the quotient of determinants

$$
\operatorname{det}\left(\begin{array}{cccc}
1 & 1 & \ldots & 1 \\
\rho_{1} & \rho_{2} & \ldots & \rho_{\ell} \\
\vdots & \vdots & \ddots & \vdots \\
\rho_{1}^{\ell-2} & \rho_{2}^{\ell-2} & \ldots & \rho_{\ell}^{\ell-2} \\
k_{1}\left(\rho_{1}\right) & k_{2}\left(\rho_{2}\right) & \ldots & k_{\ell}\left(\rho_{\ell}\right)
\end{array}\right) \operatorname{det}\left(\begin{array}{cccc}
1 & 1 & \ldots & 1 \\
\rho_{1} & \rho_{2} & \ldots & \rho_{\ell} \\
\vdots & \vdots & \ddots & \vdots \\
\rho_{1}^{\ell-2} & \rho_{2}^{\ell-2} & \ldots & \rho_{\ell}^{\ell-2} \\
\rho_{1}^{\ell-1} & \rho_{2}^{\ell-1} & \ldots & \rho_{\ell}^{\ell-1}
\end{array}\right)^{-1}
$$

(2) If $f$ is bounded on the domain $\rho_{1}<\cdots<\rho_{\ell}$, then $k_{1}=\cdots=k_{\ell}$.

(3) We have $f\left(\rho_{1}, \ldots, \rho_{\ell}\right) \equiv 0$ if and only if $k_{i}\left(\rho_{i}\right)=p\left(\rho_{i}\right)$, where $p\left(\rho_{i}\right)$ is a polynomial in $\rho_{i}$ (independent of $i$ ) of degree $\leq \ell-2$.

Proof. The proof of (1) follows from standard manipulations for calculating determinants and by applying the formula for the determinant of the Vandermonde matrix.

To prove (2), consider for instance the limit $\rho_{1} \rightarrow \rho_{2}$ under which the Vandermonde determinant in the denominator of the expression for $f$ tends to zero. Since $f$ is bounded, the determinant in the numerator must also tend to zero which implies that $k_{1}$ and $k_{2}$ are equal.

Part (3) follows immediately from the formula in part (1) and the vanishing of the determinant in the nominator.

We apply part $(2)$ of Lemma 5.12 to the function $f(\vec{\rho})$ from $(5.15)$ written in the form $(5.16)$ to obtain

Corollary 5.13. The parameters $a_{i}$ 's in (5.11), (5.12) are all equal. In other words, the functions $F_{i}$ from (5.11) coincide and take the form

$$
F_{i}(t)=F(t)=a(1-t)^{-\mathcal{C}} t^{1+\ell+\mathcal{C}}
$$

To find restrictions on the constant $\mathcal{C}$ and the number $\ell$ of non-constant eigenvalues we use another interesting property of functions of the form (5.16).

Lemma 5.14. Let

$$
f(\vec{\rho})=\sum_{i=1}^{\ell} \frac{k\left(\rho_{i}\right)}{\prod_{j \neq i}\left(\rho_{i}-\rho_{j}\right)},
$$

where $\vec{\rho}=\left(\rho_{1}, \ldots, \rho_{\ell}\right)$. If $k$ is smooth in a neighbourhood of $x \in \mathbb{R}$, then

$$
\lim _{\vec{\rho} \rightarrow \vec{x}} f(\vec{\rho})=\frac{1}{(\ell-1) !} k^{(\ell-1)}(x),
$$

where we define $\vec{x}=(x, \ldots, x)$.

Proof. In a neighbourhood of $x$, we can write $k$ in the form

$$
k(t)=\sum_{j=0}^{\ell-1} \frac{1}{j !} k^{(j)}(x)(t-x)^{j}+\mathcal{O}\left((t-x)^{\ell}\right) .
$$


Inserting this into the formula for $f$, we obtain

$$
f(\vec{\rho})=\sum_{j=0}^{\ell-1} \frac{1}{j !} k^{(j)}(x) \sum_{i=1}^{\ell} \frac{\left(\rho_{i}-x\right)^{j}}{\prod_{r \neq i}\left(\rho_{i}-\rho_{r}\right)}+\sum_{i=1}^{\ell} \frac{\mathcal{O}\left(\left(\rho_{i}-x\right)^{\ell}\right)}{\prod_{j \neq i}\left(\rho_{i}-\rho_{j}\right)} .
$$

Applying Lemma $5.12(3)$ to the functions $k_{i}\left(\rho_{i}\right)$ of the form $\left(\rho_{i}-x\right)^{j}$, we obtain

$$
f(\vec{\rho})=\frac{1}{(\ell-1) !} k^{(\ell-1)}(x)+\sum_{i=1}^{\ell} \frac{\mathcal{O}\left(\left(\rho_{i}-x\right)^{\ell}\right)}{\prod_{j \neq i}\left(\rho_{i}-\rho_{j}\right)} .
$$

The claim now follows by taking the limit $\vec{\rho} \rightarrow \vec{x}$.

The limiting behaviour $f(\vec{\rho}) \rightarrow 0$ for $\vec{\rho} \rightarrow(1, \ldots, 1)$ and $\vec{\rho} \rightarrow(0, \ldots, 0)$ (see Proposition 5.11) combined with Lemma 5.14 with $k(t)=F(t)=a(1-t)^{-\mathcal{C}} t^{1+\ell+\mathcal{C}}$ implies that the function $F(t)$ must have a zero of order $\ell-1$ at $t=0$ and $t=1$. Hence,

$$
-\mathcal{C}>\ell-1 \text { and } 1+\ell+\mathcal{C}>\ell-1
$$

or equivalently

$$
-2<\mathcal{C}<1-\ell
$$

This inequality is not fulfilled for $\ell \geq 3$. Thus, we have

Corollary 5.15. The number $\ell$ of non-constant eigenvalues is either 1 or 2 . Moreover, we have

$$
-2<\mathcal{C}<-1 \text { for } \ell=2 \quad \text { and } \quad-2<\mathcal{C}<0 \text { for } \ell=1 \text {. }
$$

Now let us show that also $\ell=2$ contradicts our assumptions. If $\ell=2$, then the metric $h=\left.g\right|_{\mathcal{L}}$ (see Proposition 5.7) takes the form

$$
\left(\rho_{1}-\rho_{2}\right)\left(\frac{\mathrm{d} \rho_{1}^{2}}{F\left(\rho_{1}\right)}-\frac{\mathrm{d} \rho_{2}^{2}}{F\left(\rho_{2}\right)}\right)
$$

where $F(t)=a(1-t)^{-\mathcal{C}} t^{3+\mathcal{C}}$ and $-2<\mathcal{C}<-1$. Without loss of generality we may assume that $a=1$.

Let us compute one special eigenvalue of the curvature operator of the metric $g$ on $M$ by using Proposition A.2, see Appendix. We know that each eigenvector of $A$ (e.g., grad $\left.\rho_{i}\right)$ is at the same time an eigenvector of $\nabla \Lambda$ (Lemma A.1 and Remark A.1). In particular, we must have

$$
\nabla_{\operatorname{grad} \rho_{i}} \Lambda=m_{i} \operatorname{grad} \rho_{i} \quad \text { for } i=1,2,
$$

where the functions $m_{1}, m_{2}$ are the eigenvalues of the endomorphism $\nabla \Lambda$ (of course, this observation is not limited to the case $\ell=2$ ). Then according to Proposition A.2 and Remark A.2, the function

$$
\lambda=\frac{m_{1}-m_{2}}{\rho_{1}-\rho_{2}}
$$

is an eigenvalue of the curvature operator.

Proposition 5.16. The eigenvalue $\lambda$ of the curvature operator is given (up to multiplication with a non-zero constant) by the formula

$$
\lambda=\frac{\left(\rho_{1}-\rho_{2}\right)\left(F^{\prime}\left(\rho_{1}\right)+F^{\prime}\left(\rho_{2}\right)\right)+2\left(F\left(\rho_{2}\right)-F\left(\rho_{1}\right)\right)}{4\left(\rho_{1}-\rho_{2}\right)^{3}},
$$

where $F(t)=(1-t)^{-\mathcal{C}} t^{3+\mathcal{C}}$.

Proof. We have $\Lambda=\frac{1}{2}\left(\operatorname{grad} \rho_{1}+\operatorname{grad} \rho_{2}\right)$, hence,

$$
\nabla_{\Lambda} \Lambda=\frac{1}{2} \nabla_{\operatorname{grad} \rho_{1}} \Lambda+\frac{1}{2} \nabla_{\operatorname{grad} \rho_{2}} \Lambda=\frac{1}{2}\left(m_{1} \operatorname{grad} \rho_{1}+m_{2} \operatorname{grad} \rho_{2}\right) .
$$

Dualizing this (and using that $\nabla \Lambda$ is $g$-selfadjoint), we obtain

$$
\mathrm{d} g(\Lambda, \Lambda)=m_{1} \mathrm{~d} \rho_{1}+m_{2} \mathrm{~d} \rho_{2} .
$$

Thus, to find the formulas for $m_{1}$ and $m_{2}$ it remains to calculate the differential of $g(\Lambda, \Lambda)$. 
By (5.17) we have

$$
g(\Lambda, \Lambda)=\frac{1}{4}\left(\left|\operatorname{grad} \rho_{1}\right|^{2}+\left|\operatorname{grad} \rho_{2}\right|^{2}\right)=\frac{1}{4} \frac{F_{1}\left(\rho_{1}\right)-F_{2}\left(\rho_{2}\right)}{\rho_{1}-\rho_{2}} .
$$

The functions $F_{i}(t)$ are given by formula (5.12) (with $\ell=2$ ) and by Corollary 5.13, they are all equal. Then,

and we obtain

$$
\begin{aligned}
\mathrm{d} g(\Lambda, \Lambda)= & \frac{1}{4\left(\rho_{1}-\rho_{2}\right)^{2}}\left[\left(\left(\rho_{1}-\rho_{2}\right) F^{\prime}\left(\rho_{1}\right)+F\left(\rho_{2}\right)-F\left(\rho_{1}\right)\right) \mathrm{d} \rho_{1}\right. \\
& \left.-\left(\left(\rho_{1}-\rho_{2}\right) F^{\prime}\left(\rho_{2}\right)+F\left(\rho_{2}\right)-F\left(\rho_{1}\right)\right) \mathrm{d} \rho_{2}\right]
\end{aligned}
$$

$$
m_{1}=\frac{\left(\rho_{1}-\rho_{2}\right) F^{\prime}\left(\rho_{1}\right)+F\left(\rho_{2}\right)-F\left(\rho_{1}\right)}{4\left(\rho_{1}-\rho_{2}\right)^{2}}
$$

and

$$
m_{2}=-\frac{\left(\rho_{1}-\rho_{2}\right) F^{\prime}\left(\rho_{2}\right)+F\left(\rho_{2}\right)-F\left(\rho_{1}\right)}{4\left(\rho_{1}-\rho_{2}\right)^{2}} .
$$

Thus, $\lambda=\left(m_{1}-m_{2}\right) /\left(\rho_{1}-\rho_{2}\right)$ is given by formula (5.18) as we claimed.

From Proposition 5.6, we also know that this number $\lambda$ can be computed for the restriction $h$ of $g$ to the totally geodesic leaves of the distribution $\mathcal{U}$, i.e. for the metric (5.17). Notice that (5.18) coincides with the usual formula for the scalar curvature of $h$. Being an eigenvalue of the curvature operator of $g$, the function $\lambda$ must be bounded on $U=\left\{0<\rho_{1}<\rho_{2}<1\right\}$.

Lemma 5.17. If $F$ is smooth at $x \in \mathbb{R}$, then the function $\lambda\left(\rho_{1}, \rho_{2}\right)$ given by (5.18) is bounded in a neighbourhood of the point $(x, x)$. Moreover,

$$
\lim _{\left(\rho_{1}, \rho_{2}\right) \rightarrow(x, x)} \lambda\left(\rho_{1}, \rho_{2}\right)=\frac{1}{24} F^{\prime \prime \prime}(x) .
$$

Conversely, if $\lim _{t \rightarrow x} F^{\prime \prime \prime}(t)=\infty$, then $\lambda$ is not bounded as $\left(\rho_{1}, \rho_{2}\right) \rightarrow(x, x)$.

Proof. Setting $y=\rho_{1}$ and $x=\rho_{2}$ in formula (5.18) for $\lambda$ and inserting the Taylor expansion at the point $x$ of $F(y)$ considered as a function of $y$, we obtain

$$
\begin{gathered}
\lim _{y \rightarrow x} \lambda(y, x)=\lim _{y \rightarrow x} \frac{(y-x)\left(F^{\prime}(y)+F^{\prime}(x)\right)+2(F(x)-F(y))}{4(y-x)^{3}} \\
=\lim _{y \rightarrow x} \frac{1}{4(y-x)^{3}}\left((y-x)\left(2 F^{\prime}(x)+F^{\prime \prime}(x)(y-x)+\frac{1}{2} F^{\prime \prime \prime}(x)(y-x)^{2}\right)\right. \\
\left.-2\left(F^{\prime}(x)(y-x)+\frac{1}{2} F^{\prime \prime}(x)(y-x)^{2}+\frac{1}{6} F^{\prime \prime \prime}(x)(y-x)^{3}\right)+\mathcal{O}\left((y-x)^{4}\right)\right) \\
=\lim _{y \rightarrow x} \frac{\frac{1}{6} F^{\prime \prime \prime}(x)(y-x)^{3}+\mathcal{O}\left((y-x)^{4}\right)}{4(y-x)^{3}}=\frac{1}{24} F^{\prime \prime \prime}(x) .
\end{gathered}
$$

Now it is easy to see that the condition that $F^{\prime \prime \prime}(t)$ is bounded as $t \rightarrow 0$ and $t \rightarrow 1$ for $F(t)=(1-t)^{-\mathcal{C}} t^{3+\mathcal{C}}$ can only be fulfilled for $\mathcal{C}=0,-1,-2,-3$ (by the way, in this case $\lambda$ is constant). But in our case, $-2<\mathcal{C}<-1$ so that $\lambda$ goes to infinity either for $\left(\rho_{1}, \rho_{2}\right) \rightarrow(1,1)$ or $\left(\rho_{1}, \rho_{2}\right) \rightarrow(0,0)$.

Thus, we conclude that $\ell=2$ is forbidden and the only remaining case is $\ell=1$. This completes the proof of Proposition 5.9.

Remark 5 for Theorem 5.1. In the proof of Proposition 5.16, we used Proposition A.2 to derive a formula for one of the eigenvalues of the curvature operator. An analogue of Proposition A.2 holds in the non-Kähler case too (see [5]) and the proof remains essentially the same. Thus, Proposition 5.16 and Lemma 5.17 remain unchanged and we obtain that the number of nonconstant eigenvalues $\ell$ is 1 (in a neighbourhood of a regular point). 
5.7. Proof of Theorem $\mathbf{1 . 1}$ when there is only one non-constant eigenvalue. We deal with the PDE system (5.8) which, in the case one single non-constant eigenvalue $\rho$ of $A$, takes the form

$$
\begin{gathered}
\mathcal{L}_{v} A=A(\operatorname{Id}-A), \\
\mathcal{L}_{v} g=-g A-(\rho+\mathcal{C}) g .
\end{gathered}
$$

Recall from Proposition 5.6 that we have two mutually orthogonal integrable distributions $\mathcal{U}$ and $\mathcal{U}^{\perp}$ on $M$, the first one $\mathcal{U}$ being 1-dimensional and totally geodesic and the metric takes the following matrix form

$$
g=\left(\begin{array}{cc}
g_{1}(\rho) & 0 \\
0 & g_{2}(\rho, y)
\end{array}\right)
$$

w.r.t. the orthogonal decomposition $T M=\mathcal{U} \oplus \mathcal{U}^{\perp}$. We also have a $c$-projective vector field $v$ preserving both $\mathcal{U}$ and $\mathcal{U}^{\perp}$, so that $v=v_{1}(\rho)+v_{2}(y)$, where $v_{1}$ and $v_{2}$ are the components of $v$ w.r.t. $\mathcal{U}$ resp. $\mathcal{U}^{\perp}$ (see also Corollary 5.8). Hence

$$
\mathcal{L}_{v} g=\left(\begin{array}{cc}
\mathcal{L}_{v_{1}} g_{1} & 0 \\
0 & \mathcal{L}_{v_{2}} g_{2}+\mathcal{D}_{v_{1}} g_{2}
\end{array}\right)
$$

where $\mathcal{D}_{v_{1}}$ means that we differentiate each term of the matrix $g_{2}$ along $v_{1}$.

Our goal is to analyse how the volume form of the metric $g_{2}$, defined on the leaves of $\mathcal{U}^{\perp}$, is changing under the flow generated by $v_{2}$. In other words, we want to compute the coefficient $f(\rho, y)$ in the formula $\mathcal{L}_{v_{2}} \operatorname{vol}_{g_{2}}=f(\rho, y) \cdot \operatorname{vol}_{g_{2}}$. We will show that this coefficient is constant. Namely,

Proposition 5.18. We have $\mathcal{L}_{v_{2}} \operatorname{vol}_{g_{2}}=(-\mathcal{C}-1)\left(m_{1}+m_{0}+1\right) \operatorname{vol}_{g_{2}}$.

Proof. By (5.19), we have $\mathcal{L}_{v} g=-g \cdot(A+(\rho+\mathcal{C}) \mathrm{Id})$. Since $A$ admits a natural splitting $\left(\begin{array}{cc}\rho & 0 \\ 0 & A_{2}\end{array}\right)$ w.r.t. $\mathcal{U}$ and $\mathcal{U}^{\perp}$, we get

$$
\mathcal{L}_{v} g=\left(\begin{array}{cc}
-\frac{1}{F}(2 \rho+\mathcal{C}) & 0 \\
0 & -g_{2}\left(A_{2}+(\rho+\mathcal{C}) \mathrm{Id}\right)
\end{array}\right)
$$

Hence, comparing with $(5.20)$, we obtain $\mathcal{L}_{v_{2}} g_{2}=-g_{2}\left(A_{2}+(\rho+\mathcal{C}) \mathrm{Id}\right)-\mathcal{D}_{v_{1}} g_{2}$.

We now use the following general formula that explains the relation between $\mathcal{L}_{v_{2}} g_{2}$ and $\mathcal{L}_{v_{2}} \operatorname{vol}_{g_{2}}$ :

$$
\mathcal{L}_{v_{2}} \operatorname{vol}_{g_{2}}=f \cdot \operatorname{vol}_{g_{2}}, \quad \text { where } f=\frac{1}{2} \operatorname{tr}\left(g_{2}^{-1} \mathcal{L}_{v_{2}} g_{2}\right) .
$$

Hence in our case

$$
f=-\frac{1}{2} \operatorname{tr}\left(A_{2}+(\rho+\mathcal{C}) \operatorname{Id}+g_{2}^{-1} \mathcal{D}_{v_{1}} g_{2}\right) .
$$

Let us compute $\operatorname{tr}\left(g_{2}^{-1} \mathcal{D}_{v_{1}} g_{2}\right)$. In our case $v_{1}=\rho(1-\rho) \frac{\partial}{\partial \rho}$ and the metric $g_{2}$ has the form

$$
F(\rho) \theta^{2}+g_{\mathrm{c}}\left(\left(A_{\mathrm{c}}-\rho \cdot \mathrm{Id}\right) \cdot, \cdot\right),
$$

hence, $\mathcal{D}_{v_{1}} g_{2}=\rho(1-\rho) F^{\prime}(\rho) \theta^{2}-\rho(1-\rho) g_{\mathrm{c}}(\cdot, \cdot)$. We may think of $g_{2}$ as a block diagonal form, then $\mathcal{D}_{v_{1}} g_{2}$ is block-diagonal too so that we can write it as $D_{v_{1}} g_{2}=g_{2} C$, where $C$ is an endomorphism with the matrix

$$
C=\left(\begin{array}{cc}
\rho(1-\rho) \frac{F^{\prime}(\rho)}{F(\rho)} & 0 \\
0 & -\rho(1-\rho)\left(A_{\mathrm{c}}-\rho\right)^{-1}
\end{array}\right)
$$

Therefore we have the following formula:

$$
\operatorname{tr} g_{2}^{-1} \mathcal{D}_{v_{1}} g_{2}=\operatorname{tr} C=\rho(1-\rho) \frac{\partial \ln F}{\partial \rho}-\rho(1-\rho) \operatorname{tr}\left(A_{\mathrm{c}}-\rho\right)^{-1} .
$$

The first term can be easily computed from the explicit formula for $F$. The second term is easy to find as we know that the eigenvalues of $A_{\mathrm{c}}$ are 0 and 1 with multiplicities $2 m_{0}$ and $2 m_{1}$ respectively. We get

$\operatorname{tr} g_{2}^{-1} \mathcal{D}_{v_{1}} g_{2}=(2+\mathcal{C}-2 \rho)-\rho(1-\rho)\left(-2 m_{0} \rho^{-1}+2 m_{1}(1-\rho)^{-1}\right)=2+\mathcal{C}+2 m_{0}-\rho\left(2 m_{0}+2 m_{1}+2\right)$ 
The matrix of $A_{2}$ is known, namely $A_{2}=\left(\begin{array}{cc}\rho & 0 \\ 0 & A_{\mathrm{c}}\end{array}\right)$. So $\operatorname{tr} A_{2}=\rho+\operatorname{tr} A_{\mathrm{c}}=\rho+2 m_{1}$. Thus, finally

$$
\begin{aligned}
f= & -\frac{1}{2}\left(\rho+2 m_{1}+(\rho+\mathcal{C})\left(2 m_{1}+2 m_{0}+1\right)+2+\mathcal{C}+2 m_{0}-\rho\left(2 m_{0}+2 m_{1}+2\right)\right)= \\
& (-\mathcal{C}-1)\left(m_{0}+m_{1}+1\right),
\end{aligned}
$$

as claimed.

This proposition immediately implies that $\mathcal{C}=-1$. Indeed, consider $M_{\rho}=\{\rho=c\}$ where $c \in(0,1)$. Then $M_{\rho}$ is a compact smooth submanifold of $M$ which entirely belongs to the set of regular points $M^{0}$ and, therefore, the formula from Proposition 5.18 holds for the volume form on $M_{\rho}$ as a whole. However, due to compactness of $M_{\rho}$, this is impossible unless $-\mathcal{C}-1=0$. Thus, we have completely reconstructed the "non-constant" block of the metric $g$ and now we can rewrite $g$ as follows:

$$
g=F(\rho)^{-1} \mathrm{~d} \rho^{2}+F(\rho) \theta^{2}+g_{\mathrm{c}}\left(\left(A_{\mathrm{c}}-\rho \mathrm{Id}\right) \cdot, \cdot\right),
$$

where $F(\rho)=-4 B(1-\rho) \rho$ and $B \neq 0$ is some constant (the notation $-4 B$ for the constant factor is chosen to emphasize the relationship with some formulas from [19] that are used at the final stage of our proof).

Remark 6 for Theorem 5.1. The proof of and the formula in Proposition 5.18 slightly changes because now the terms with $\theta$ do not appear. Here is the modified version in the projective setting:

Proposition 5.19. We have $\mathcal{L}_{v_{2}} \operatorname{vol}_{g_{2}}=\frac{1}{2}(-\mathcal{C}-1)\left(m_{1}+m_{0}\right) \operatorname{vol}_{g_{2}}$.

Proof. By (5.19), we have $\mathcal{L}_{v} g=-g \cdot(A+(\rho+\mathcal{C}) \mathrm{Id})$. Since $A$ admits a natural splitting $\left(\begin{array}{cc}\rho & 0 \\ 0 & A_{2}\end{array}\right)$ w.r.t. $\mathcal{U}$ and $\mathcal{U}^{\perp}$, we get

$$
\mathcal{L}_{v} g=\left(\begin{array}{cc}
-\frac{1}{F}(2 \rho+\mathcal{C}) & 0 \\
0 & -g_{2}\left(A_{2}+(\rho+\mathcal{C}) \mathrm{Id}\right)
\end{array}\right)
$$

Hence, comparing with $(5.20)$, we obtain $\mathcal{L}_{v_{2}} g_{2}=-g_{2}\left(A_{2}+(\rho+\mathcal{C}) \mathrm{Id}\right)-\mathcal{D}_{v_{1}} g_{2}$. Using

$$
\mathcal{L}_{v_{2}} \operatorname{vol}_{g_{2}}=f \cdot \operatorname{vol}_{g_{2}}, \quad \text { where } f=\frac{1}{2} \operatorname{tr}\left(g_{2}^{-1} \mathcal{L}_{v_{2}} g_{2}\right),
$$

we obtain in our case

$$
f=-\frac{1}{2} \operatorname{tr}\left(A_{2}+(\rho+\mathcal{C}) \operatorname{Id}+g_{2}^{-1} \mathcal{D}_{v_{1}} g_{2}\right) .
$$

Let us compute $\operatorname{tr}\left(g_{2}^{-1} \mathcal{D}_{v_{1}} g_{2}\right)$. In our case $v_{1}=\rho(1-\rho) \frac{\partial}{\partial \rho}$ and the metric $g_{2}$ has the form $g_{2}(\cdot, \cdot)=g_{\mathrm{c}}\left(\left(A_{\mathrm{c}}-\rho \cdot \mathrm{Id}\right) \cdot, \cdot\right)$, hence,

$$
\mathcal{D}_{v_{1}} g_{2}=-\rho(1-\rho) g_{\mathrm{c}}(\cdot, \cdot)
$$

Thus we can write $\mathcal{D}_{v_{1}} g_{2}$ as $D_{v_{1}} g_{2}=g_{2} C$, where $C$ is an endomorphism with the matrix

$$
C=-\rho(1-\rho)\left(A_{\mathrm{c}}-\rho\right)^{-1}
$$

Therefore we have the following formula:

$$
\operatorname{tr} g_{2}^{-1} \mathcal{D}_{v_{1}} g_{2}=\operatorname{tr} C=-\rho(1-\rho) \operatorname{tr}\left(A_{\mathrm{c}}-\rho\right)^{-1} .
$$

This quantity is easy to find as we know the eigenvalues of $A_{\mathrm{c}}$ are 0 and 1 with multiplicities $m_{0}$ and $m_{1}$ respectively. We get

$$
\operatorname{tr} g_{2}^{-1} \mathcal{D}_{v_{1}} g_{2}=-\rho(1-\rho)\left(-m_{0} \rho^{-1}+m_{1}(1-\rho)^{-1}\right)=m_{0}(1-\rho)-m_{1} \rho
$$

The matrix of $A_{2}$ coincides with $A_{\mathrm{c}}$ so that $\operatorname{tr} A_{2}=\operatorname{tr} A_{\mathrm{c}}=m_{1}$. Thus, finally

$$
f=-\frac{1}{2}\left(m_{1}+(\rho+\mathcal{C})\left(m_{1}+m_{0}\right)+m_{0}(1-\rho)-m_{1} \rho\right)=\frac{1}{2}(-\mathcal{C}-1)\left(m_{0}+m_{1}\right),
$$

as claimed. 
The conclusion from this proposition remains unchanged: $\mathcal{C}=-1$ as required. We use the fact that the leaves of $\mathcal{U}^{\perp}$ are smooth closed submanifolds of the form $\{\rho=c\}$ which are entirely located in the set of regular points. Since the leaves of $\mathcal{U}^{\perp}$ are common levels of non-constant eigenvalues, the latter follows from the fact (used already several times) that $0<\rho<1$ implies $\mathrm{d} \rho(p) \neq 0$. Hence we have the following formula for the metric (this is (5.21) with the term with $\theta$ removed):

$$
g=F(\rho)^{-1} \mathrm{~d} \rho^{2}+g_{\mathrm{c}}\left(\left(A_{\mathrm{c}}-\rho \mathrm{Id}\right) \cdot, \cdot\right),
$$

where $F(\rho)=-4 B(1-\rho) \rho, B \neq 0$ is some constant and $A_{\mathrm{c}}$ is parallel w.r.t. $g_{\mathrm{c}}$.

Denote by $E_{0}$ resp. $E_{1}$ the generalised eigenspaces of $A$ corresponding to the eigenvalues 0 resp. 1. Let $L_{0}=\left.A\right|_{E_{0}}$ and $L_{1}=\left.A\right|_{E_{1}}$ denote the restrictions of $A$ to these subspaces. We start with describing $\nabla \Lambda$ restricted to $E_{0}$ resp. $E_{1}$ explicitly as a matrix function of $L_{0}$ resp. $L_{1}$. In fact, it is a general statement (see Remark A.1 in the appendix), that $\nabla \Lambda$ and $A$ commute and, moreover, at each point $\nabla \Lambda$ can be written as a function (or even polynomial) of $A$.

Lemma 5.20. At each regular point, we have

$$
\left.\nabla \Lambda\right|_{E_{0} \oplus E_{1}}=-\left.g(\Lambda, \Lambda)(A-\rho \mathrm{Id})^{-1}\right|_{E_{0} \oplus E_{1}}=\left.B(1-\rho) \rho(A-\rho \mathrm{Id})^{-1}\right|_{E_{0} \oplus E_{1}} .
$$

Proof. In the special case of only one non-constant eigenvalue $\rho, \Lambda=\frac{1}{2} \operatorname{grad} \rho$ itself is an eigenvector field of $A$ corresponding to the eigenvalue $\rho$. Hence, we can apply (2.4) with $X$ replaced by $\Lambda$. Then (2.4) becomes

$$
(A-\rho \mathrm{Id}) \nabla_{Y} \Lambda=-g(\Lambda, \Lambda) Y
$$

for any tangent vector $Y \in E_{0} \oplus E_{1}$, or equivalently, if we take into account that $E_{0} \oplus E_{1}$ is invariant under $A-\rho \cdot \operatorname{Id}$ and $\left.(A-\rho \cdot \operatorname{Id})\right|_{E_{0} \oplus E_{1}}$ is invertible:

$$
\left.\nabla \Lambda\right|_{E_{0} \oplus E_{1}}=-\left.g(\Lambda, \Lambda)(A-\rho \mathrm{Id})^{-1}\right|_{E_{0} \oplus E_{1}} .
$$

It remains to notice that $(5.21)$ implies $g(\Lambda, \Lambda)=\frac{1}{4} g(\operatorname{grad} \rho, \operatorname{grad} \rho)=B(1-\rho) \rho$, as stated.

Lemma 5.21. At each regular point, we have $\left.A\right|_{E_{0}}=L_{0}=0$ and $\left.A\right|_{E_{1}}=L_{1}=\mathrm{Id}$.

Proof. Our statement is equivalent to the absence of non-trivial Jordan blocks corresponding to the constant eigenvalues 0 and 1 . By contradiction, assume that non-trivial Jordan blocks exist and apply Proposition A.2 to compute the eigenvalues of the curvature operator of $g$ related to these blocks.

Formula (5.23) expresses $\left.\nabla \Lambda\right|_{E_{0} \oplus E_{1}}$ (pointwise) as a matrix function $f\left(\left.A\right|_{E_{0} \oplus E_{1}}\right)$, where $f(t)=$ $B(1-\rho) \rho(t-\rho)^{-1}$. Then according to Proposition A.2 and Remark A.2 from Appendix, we conclude that if $L_{0}$ (resp. $L_{1}$ ) has a non-trivial Jordan block, then

$$
4 f^{\prime}(0)=-4 B \frac{1-\rho}{\rho} \quad\left(\text { resp. } 4 f^{\prime}(1)=-4 B \frac{\rho}{1-\rho}\right)
$$

is an eigenvalue of the curvature operator $R$ of $(M, g, J)$. When restricted to a non-constant integral curve of $v$, this eigenvalue goes to infinity for $t \rightarrow-\infty$ resp. $t \rightarrow+\infty$ which contradicts to the boundedness of the eigenvalues of $R$. Thus, we conclude $L_{0}=0$ and $L_{1}=\mathrm{Id}$.

Remark 7 for Theorem 5.1. Lemma 5.20 uses formula (2.4) which, in the pseudo-Riemannian case takes the form:

$$
(A-\rho \mathrm{Id}) \nabla_{Y} X=\mathrm{d} \rho(Y) X-g(X, Y) \Lambda-g(X, \Lambda) Y
$$

where $\Lambda=\frac{1}{2} \operatorname{grad} \operatorname{tr} A=\frac{1}{2} \operatorname{grad} \rho, X$ is a vector field satisfying $(A-\rho \mathrm{Id}) X=0$, i.e., a $\rho$-eigenvector field and $Y$ is an arbitrary vector field.

Notice that in this case $\Lambda$ satisfies the condition for $X$, so in this formula we may set $X=\Lambda$. Then we get

$$
(A-\rho \mathrm{Id}) \nabla_{Y} \Lambda=2 g(\Lambda, Y) \Lambda-g(\Lambda, \Lambda) Y-g(Y, \Lambda) \Lambda=g(\Lambda, Y) \Lambda-g(\Lambda, \Lambda) Y,
$$

and if we assume that $Y \in E_{0} \oplus E_{1}$ and take into account that $\Lambda$ is orthogonal to $E_{0} \oplus E_{1}$, we obtain the same formula as in the Kähler case:

$$
(A-\rho \mathrm{Id}) \nabla_{Y} \Lambda=-g(\Lambda, \Lambda) Y .
$$


The rest of the proof does not change and therefore, in the projective setting, Lemmas 5.20 and 5.21 remain unchanged.

Lemma 5.22. At every point, we have

$$
\nabla \Lambda=\mu \mathrm{Id}+B A .
$$

for the constant $B$ and a function $\mu=B(\rho-1)$. Moreover,

$$
\nabla \mu=2 B \Lambda^{b} .
$$

Proof. We will first prove the lemma near a regular point. We know that the eigenspaces of $A$ are invariant under $\nabla \Lambda$ and from Lemma 5.20, we know the formula for the restriction of $\nabla \Lambda$ onto $E_{0} \oplus E_{1}$. Taking into account Lemma 5.21 we obtain

$$
\left.\nabla \Lambda\right|_{E_{0}}=B(1-\rho) \rho(-\rho \mathrm{Id})^{-1}=B(\rho-1) \operatorname{Id}=\left.(\mu \mathrm{Id}+B A)\right|_{E_{0}},
$$

and

$$
\left.\nabla \Lambda\right|_{E_{1}}=B(1-\rho) \rho(\mathrm{Id}-\rho \mathrm{Id})^{-1}=B \rho \mathrm{Id}=\left.(\mu \mathrm{Id}+B A)\right|_{E_{1}},
$$

Thus, it remains to verify the formula for the restriction of $\nabla \Lambda$ onto the two-dimensional $\rho$ eigenspace $E_{\rho}$.

Notice that $\Lambda \in E_{\rho}$ and moreover $\Lambda$ is the tangent vector to the one-dimensional totally geodesic distribution $\mathcal{U}$. In other words, we can consider $\Lambda$ as a tangent vector to a geodesic $\gamma(t)$. Hence, $(\nabla \Lambda) \Lambda=\nabla_{\Lambda} \Lambda=f \Lambda$ where $f=\frac{1}{2} \frac{\Lambda(g(\Lambda, \Lambda))}{g(\Lambda, \Lambda)}$.

Using $\Lambda=\frac{1}{2} \operatorname{grad} \rho$ and the explicit formula (5.21) for $g$, we obtain

$$
f=B(2 \rho-1) .
$$

The other $\rho$-eigenvector is $J \Lambda$. Since $\Lambda$ and $J \Lambda$ commute, we get

$$
(\nabla \Lambda) J \Lambda=\nabla_{J \Lambda} \Lambda=\nabla_{\Lambda} J \Lambda=J \nabla_{\Lambda} \Lambda=J(B(2 \rho-1) \Lambda)=B(2 \rho-1) J \Lambda .
$$

Thus,

$$
\left.\nabla \Lambda\right|_{E_{\rho}}=B(2 \rho-1) \operatorname{Id}=\left.(\mu \mathrm{Id}+B A)\right|_{E_{\rho}} .
$$

Since at regular points $T M$ decomposes as $T M=E_{\rho} \oplus E_{0} \oplus E_{1}$, we have verified equation (5.24) in a neighbourhood of every point of a dense and open subset of $M$ for a local constant $B$ and a locally defined function $\mu=B(\rho-1)$. Taking the derivative of this function (and using $\left.\mathrm{d} \rho=2 \Lambda^{\mathrm{b}}\right)$ shows that it satisfies $(5.25)$. It was proven in $[19, \S 2.5]$ that having the equations $(5.24)$ and (5.25) satisfied in a neighbourhood of almost every point for locally defined constant $B$ and function $\mu$, the constant $B$ is the same for each such neighbourhood, hence, is globally defined, and therefore also $\mu$ is globally defined. This completes the proof of the lemma.

Remark 8 for Theorem 5.1. The statement of Lemma 5.22 remains unchanged. The proof of Lemma 5.22 changes slightly, as the $\rho$-eigenspace is one-dimensional (not two-dimensional as it was in the c-projective setting). This makes the proof shorter as we do not need to consider the second eigenvector $J \Lambda$. The projective analogue of $[19, \S 2.5]$ is $[21, \S 2.3 .4]$.

Now we can proof Theorem 1.1. We have shown that the existence of a non-affine c-projective vector field on a closed connected Kähler manifold $(M, g, J)$ of arbitrary signature implies the existence of a solution $(A, \Lambda, \mu)$ of the system

$$
\begin{gathered}
\nabla_{X} A=X^{\mathrm{b}} \otimes \Lambda+\Lambda^{\mathrm{b}} \otimes X+(J X)^{\mathrm{b}} \otimes J \Lambda+(J \Lambda)^{\mathrm{b}} \otimes J X \\
\nabla \Lambda=\mu \mathrm{Id}+B A, \\
\nabla \mu=2 B \Lambda^{\mathrm{b}}
\end{gathered}
$$

for a certain constant $B$. This solution is non-trivial in the sense that $\Lambda$ is not identically zero. By [19, Theorem 7], $g$ is positive definite, up to multiplying the metric with a constant. On the other hand, Theorem 1.1 was proven for positive signature in [31]. This completes the proof of Theorem 1.1. 
Remark 9 for Theorem 5.1. The existence of $(L, \Lambda, \mu)$ satisfying (1.7) and (5.24) immediately implies that the (non-constant) function $\alpha=\operatorname{tr}(L)$ satisfies the equation

$$
\nabla^{3} \alpha(X, Y, Z)-B \cdot(2(\nabla \alpha \otimes g)(X, Y, Z)+(\nabla \alpha \otimes g)(Y, X, Z)+(\nabla \alpha \otimes g)(Z, X, Y))=0,
$$

see e.g. [21, Corollary 4]. By [27, Theorem 1], the metric $-B g$ is positive definite. This contradiction proves Theorem 5.1.

\section{Final step of the proof of Theorem 1.2: the Case of a non-constant Jordan BLOCK}

In $\S 5$, the Lichnerowicz conjecture was proved under the additional assumption that the nonconstant part of $A$ is diagonalisable.

In the case when the endomorphism $A$ has a non-trivial Jordan block with a non-constant eigenvalue, the scheme of the proof remains essentially the same but we need to modify some steps accordingly. In fact, the proof becomes much easier because the presence of a Jordan block, as we shall see below, immediately leads to unboundedness of one special eigenvalue of the curvature operator.

The first steps of the proof do not use the algebraic type of $A$ and, therefore, they remain the same as in Sections 5.3 and 5.4. Namely, we may assume that the degree of mobility of $g$ equals 2 and $g, A$ and $v$ satisfy the equations

$$
\begin{aligned}
\mathcal{L}_{v} A & =-A^{2}+A, \\
\mathcal{L}_{v} g & =-g A-\left(\operatorname{tr} A+\mathcal{C}^{\prime}\right) g .
\end{aligned}
$$

These equations coincide with (5.8) though we need to replace $\sum \rho_{i}$ by $\operatorname{tr} A$ as some non-constant eigenvalues may now have multiplicity $>1$, see also our comment on (5.8) in Remark 4 for Theorem 5.1.

All the eigenvalues of $A$ are real and satisfy the equation $\mathcal{L}_{v} \rho=-\rho^{2}+\rho$, in particular, there are at most two constant eigenvalues, 0 and 1 , and, due to boundedness of the non-constant eigenvalues, they satisfy $0 \leq \rho_{i} \leq 1$.

The definition of the set $M^{0}$ of regular points also remains the same but the algebraic type of $A$ changes. Recall that in general $M^{0}$ may consist of several connected components with different algebraic types, so we continue working with one of them (we still denote it by $M^{0}$ and refer to it as the set of regular points). In the Lorentzian case, two sizes of Jordan blocks for $g$-selfadjoint endomorphisms are allowed, $2 \times 2$ and $3 \times 3$. Thus, to complete the proof of Theorem 1.2 we need to consider two additional types of regular points $p \in M^{0}$.

Namely, below we assume that the endomorphism $A$ has $\ell$ non-constant distinct real eigenvalues $\rho_{1}, \ldots, \rho_{\ell}$ and, possibly, two constant eigenvalues 0 and 1 of multiplicity $m_{0}$ and $m_{1}$ respectively. The first eigenvalue $\rho_{1}$ has multiplicity 2 or 3 , and the endomorphism $A$ "contains" a single $2 \times 2$ or resp. $3 \times 3$ Jordan $\rho_{1}$-block. On $M^{0}$, the eigenvalues $\rho_{i}$ 's are ordered:

$$
0<\rho_{2}<\cdots<\rho_{\ell}<1 \text { and } \rho_{1} \text { belongs to one of the intervals }\left(0, \rho_{2}\right),\left(\rho_{2}, \rho_{3}\right), \ldots,\left(\rho_{\ell}, 1\right) .
$$

Due to the existence of the projective vector field $v$, the above conditions automatically imply that $\mathrm{d} \rho_{i} \neq 0$ on $M^{0}$.

In a neighbourhood of any point $p \in M^{0}$ we can now, following [9], find a canonical coordinate system and reduce $g$ and $A$ to a normal form. In general, this normal form contains arbitrary functions $F_{i}\left(\rho_{i}\right)$. The existence of a projective vector field $v$ on $M^{0}$, satisfying (6.1) allows us to reconstruct these functions (as well as the components of $v$ ) almost uniquely, i.e. up to a finite number of arbitrary constants of integration (cf. Proposition 5.7). This "reconstruction" can be done by a straightforward computation as in Proposition 5.7, but since now we deal with a more complicated situation involving Jordan blocks, we prefer to use the following general statement which explains how to split (6.1) in a block-wise manner. This statement is a direct corollary of the splitting construction from [8]. 
Theorem 6.1. Let $(h, L)$ be a compatible pair on $M$ which splits into two blocks in the sense of $[8,9]$, i.e., there exist a local coordinate system $x, y$ with $x=\left(x_{1}, \ldots, x_{n_{1}}\right)$ and $y=\left(y_{1}, \ldots, y_{n_{2}}\right)$ and compatible pairs $\left(h_{1}(x), L_{1}(x)\right)$ and $\left(h_{2}(y), L_{2}(y)\right)$ such that

$$
h(x, y)=\left(\begin{array}{cc}
h_{1}(x) \chi_{L_{2}}\left(L_{1}(x)\right) & 0 \\
0 & h_{2}(y) \chi_{L_{1}}\left(L_{2}(y)\right)
\end{array}\right), \quad L(x, y)=\left(\begin{array}{cc}
L_{1}(x) & 0 \\
0 & L_{2}(y)
\end{array}\right)
$$

where $\chi_{L_{1}}(\cdot)$ and $\chi_{L_{2}}(\cdot)$ denote the characteristic polynomials of the blocks $L_{1}$ and $L_{2}$ respectively. Let $v$ be a projective vector field for $g$ satisfying the equations

$$
\begin{aligned}
\mathcal{L}_{v} L & =-L^{2}+L, \\
\mathcal{L}_{v} h & =-h L-(\operatorname{tr} L+\mathcal{C}) h .
\end{aligned}
$$

Then the vector field $v$ and equations (6.3) also split as follows:

$$
v(x, y)=v_{1}(x)+v_{2}(y)
$$

where $v_{i}$ denote the natural projections of $v$ on the $x$-and $y$-subspaces and (6.3) is equivalent to

$$
\begin{array}{ll}
\mathcal{L}_{v_{1}} L_{1}=-L_{1}^{2}+L_{1}, & \mathcal{L}_{v_{1}} h_{1}=\left(n_{2}-1\right) h_{1} L_{1}-\left(\operatorname{tr} L_{1}+\mathcal{C}+n_{2}\right) h_{1}, \\
\mathcal{L}_{v_{2}} L_{2}=-L_{2}^{2}+L_{2}, & \mathcal{L}_{v_{2}} h_{2}=\left(n_{1}-1\right) h_{2} L_{2}-\left(\operatorname{tr} L_{2}+\mathcal{C}+n_{1}\right) h_{2} .
\end{array}
$$

Proof. The first part $v(x, y)=v_{1}(x)+v_{2}(y)$ follows from the fact that $v$ preserves the invariant $x$ - and $y$-subspaces of $L$ (see also [8, Lemma 3]). The latter can be seen from the equation $\mathcal{L}_{v} L=-L^{2}+L$. The formulas for $\mathcal{L}_{v_{1}} L_{1}$ and $\mathcal{L}_{v_{2}} L_{2}$ are straightforward. After this we can differentiate $h$ as follows:

$$
\mathcal{L}_{v_{1}+v_{2}}\left(\begin{array}{cc}
h_{1} \chi_{L_{2}}\left(L_{1}\right) & 0 \\
0 & h_{2} \chi_{L_{1}}\left(L_{2}\right)
\end{array}\right)=\left(\begin{array}{cc}
\mathcal{L}_{v_{1}}\left(h_{1} \chi_{L_{2}}\left(L_{1}\right)\right)+h_{1}\left(\mathcal{D}_{v_{2}} \chi_{L_{2}}\right)\left(L_{1}\right) & 0 \\
0 & \mathcal{L}_{v_{2}}\left(h_{2} \chi_{L_{1}}\left(L_{2}\right)\right)+h_{2}\left(\mathcal{D}_{v_{1}} \chi_{L_{1}}\right)\left(L_{2}\right)
\end{array}\right)
$$

where $\mathcal{D}_{v_{i}} \chi_{L_{i}}$ means that we differentiate each coefficient of the characteristic polynomial $\chi_{L_{i}}$ along $v_{i}$ in the usual sense. We can rewrite the right-hand side as ${ }^{5}$

$$
\begin{gathered}
\mathcal{L}_{v_{1}}\left(h_{1} \chi_{L_{2}}\left(L_{1}\right)\right)+h_{1}\left(\mathcal{D}_{v_{2}} \chi_{L_{2}}\right)\left(L_{1}\right)= \\
\left(\mathcal{L}_{v_{1}} h_{1}\right) \chi_{L_{2}}\left(L_{1}\right)+h_{1} \chi_{L_{2}}\left(L_{1}\right) \mathcal{L}_{v_{1}}\left(\ln \left(\chi_{L_{2}}\left(L_{1}\right)\right)\right)+h_{1} \chi_{L_{2}}\left(L_{1}\right) \mathcal{D}_{v_{2}}\left(\ln \left(\chi_{L_{2}}\left(L_{1}\right)\right)\right) .
\end{gathered}
$$

On the other hand, from (6.3) we know that this expression equals $h_{1} \chi_{L_{2}}\left(L_{1}\right)\left(-L_{1}-\operatorname{tr} L \cdot \operatorname{Id}-\mathcal{C} \cdot \mathrm{Id}\right)$. Multiplying by $\chi_{L_{2}}\left(L_{1}\right)^{-1}$ we get

$$
\mathcal{L}_{v_{1}} h_{1}=-h_{1}\left(\mathcal{L}_{v_{1}}\left(\ln \left(\chi_{L_{2}}\left(L_{1}\right)\right)\right)+\mathcal{D}_{v_{2}}\left(\ln \left(\chi_{L_{2}}\left(L_{1}\right)\right)\right)+L_{1}+\operatorname{tr} L \cdot \operatorname{Id}+\mathcal{C} \cdot \operatorname{Id}\right) .
$$

To evaluate this further, let $\lambda_{1}, \ldots, \lambda_{n_{2}}$ denote the eigenvalues of $L_{2}$, i.e. the roots of $\chi_{L_{2}}$ (some of them may coincide). Then,

$$
\begin{aligned}
& \mathcal{L}_{v_{1}}\left(\ln \left(\chi_{L_{2}}\left(L_{1}\right)\right)\right)=\mathcal{L}_{v_{1}}\left(\sum_{i=1}^{n_{2}} \ln \left(L_{1}-\lambda_{i} \cdot \mathrm{Id}\right)\right)=\sum_{i=1}^{n_{2}}\left(L_{1}-\lambda_{i} \cdot \mathrm{Id}\right)^{-1} \mathcal{L}_{v_{1}} L_{1}=\sum_{i=1}^{n_{2}}\left(L_{1}-\lambda_{i} \cdot \operatorname{Id}\right)^{-1}\left(-L_{1}^{2}+L_{1}\right) \\
& \mathcal{D}_{v_{2}}\left(\ln \left(\chi_{L_{2}}\left(L_{1}\right)\right)\right)=\mathcal{D}_{v_{2}}\left(\sum_{i=1}^{n_{2}} \ln \left(L_{1}-\lambda_{i} \cdot \mathrm{Id}\right)\right)=-\sum_{i=1}^{n_{2}}\left(L_{1}-\lambda_{i} \cdot \mathrm{Id}\right)^{-1} \mathcal{D}_{v_{2}} \lambda_{i}=-\sum_{i=1}^{n_{2}}\left(L_{1}-\lambda_{i} \cdot \operatorname{Id}\right)^{-1}\left(-\lambda_{i}^{2}+\lambda_{i}\right)
\end{aligned}
$$

Hence,

$$
\begin{aligned}
\mathcal{L}_{v_{1}}\left(\ln \left(\chi_{L_{2}}\left(L_{1}\right)\right)\right)+ & \left.\mathcal{D}_{v_{2}}\left(\ln \left(\chi_{L_{2}}\left(L_{1}\right)\right)\right)=\sum_{i=1}^{n_{2}}\left(L_{1}-\lambda_{i} \cdot \operatorname{Id}\right)^{-1}\left(-L_{1}^{2}+L_{1}+\lambda_{i}^{2} \cdot \operatorname{Id}-\lambda_{i} \cdot \operatorname{Id}\right)\right)= \\
& =\sum_{i=1}^{n_{2}}\left(\operatorname{Id}-\lambda_{i} \cdot \operatorname{Id}-L_{1}\right)=n_{2} \cdot \operatorname{Id}-\operatorname{tr} L_{2} \cdot \operatorname{Id}-n_{2} L_{1} .
\end{aligned}
$$

Finally, we obtain

$\mathcal{L}_{v_{1}} h_{1}=-h_{1}\left(n_{2} \cdot \mathrm{Id}-\operatorname{tr} L_{2} \cdot \mathrm{Id}-n_{2} L_{1}+L_{1}+\operatorname{tr} L \cdot \operatorname{Id}+\mathcal{C} \cdot \operatorname{Id}\right)=-h_{1}\left(\left(1-n_{2}\right) L_{1}+\left(\operatorname{tr} L_{1}+\mathcal{C}+n_{2}\right) \mathrm{Id}\right)$

\footnotetext{
${ }^{5}$ To make some expressions shorter, we are using the formula $\mathcal{L}_{v}(\ln B)=B^{-1} \mathcal{L}_{v} B$ which, in the matrix case, holds true if $B$ and $\mathcal{L}_{v} B$ commute. In our case this condition is fulfilled for $B=L_{1}$ and, consequently, for $B=\chi_{L_{2}}\left(L_{1}\right)$ since $\mathcal{L}_{v_{1}} L_{1}=-L_{1}^{2}+L_{1}$.
} 
as we claimed.

Let us apply the theorem by specifying the blocks into which we want to split:

Corollary 6.2. Let $(h, L)$ be a compatible pair that splits into two blocks of compatible pairs $\left(h_{1}, L_{1}\right),\left(h_{2}, L_{2}\right)$ of dimensions $n_{1}$ and $n_{2}$ respectively as in Theorem 6.1 . Suppose $v$ is a projective vector field such that $h, L$ and $v$ satisfy (6.3).

(1) Case of a trivial $1 \times 1$ Jordan $\rho$-block. Let $\left(h_{1}, L_{1}\right)$ be given by

$$
h_{1}=\frac{1}{F(\rho)} \mathrm{d} \rho^{2}, \quad L_{1}=\rho \frac{\partial}{\partial \rho} \otimes \mathrm{d} \rho,
$$

w.r.t. a coordinate $\rho$. Then $v_{1}=\rho(1-\rho) \frac{\partial}{\partial \rho}$ and

$$
F=a(1-\rho)^{-\mathcal{C}} \rho^{n+1+\mathcal{C}} .
$$

(2) Case of a $2 \times 2$ Jordan $\rho$-block. Let $\left(h_{1}, L_{1}\right)$ be given by

$$
h_{1}=\left(\begin{array}{cc}
0 & F(\rho)+x \\
F(\rho)+x & 0
\end{array}\right) \text { and } L_{1}=\left(\begin{array}{cc}
\rho & F(\rho)+x \\
0 & \rho
\end{array}\right)
$$

w.r.t. coordinates $x, \rho$. Then $v_{1}=G(x, \rho) \frac{\partial}{\partial x}+\rho(1-\rho) \frac{\partial}{\partial \rho}$, where

$$
G(x, \rho)=\frac{1}{2}\left(\left(n_{2}-1\right) \rho-1-\mathcal{C}-n_{2}\right) x+G_{1}(\rho)
$$

and $F(\rho), G_{1}(\rho)$ satisfy the ODE system

$$
\begin{aligned}
F^{\prime} & =\frac{1}{\rho(1-\rho)}\left(\frac{1}{2}\left(\left(n_{2}-1\right) \rho-1-\mathcal{C}-n_{2}\right) F-G_{1}\right), \\
G_{1}^{\prime} & =\frac{1}{2}\left(n_{2}-1\right) F .
\end{aligned}
$$

(3) Case of a $3 \times 3$ Jordan $\rho$-block. Let $\left(h_{1}, L_{1}\right)$ be given by

$$
h_{1}=\left(\begin{array}{ccc}
0 & 0 & F(\rho)+2 x_{2} \\
0 & 1 & x_{1} \\
F(\rho)+2 x_{2} & x_{1} & x_{1}^{2}
\end{array}\right) \text { and } L_{1}=\left(\begin{array}{ccc}
\rho & 1 & x_{1} \\
0 & \rho & F(\rho)+2 x_{2} \\
0 & 0 & \rho
\end{array}\right)
$$

in coordinates $x_{1}, x_{2}, \rho$. Then,

$$
v_{1}=G\left(x_{1}, x_{2}, \rho\right) \frac{\partial}{\partial x_{1}}+H\left(x_{2}, \rho\right) \frac{\partial}{\partial x_{2}}+\rho(1-\rho) \frac{\partial}{\partial \rho},
$$

where

$$
G\left(x_{1}, x_{2}, \rho\right)=-\frac{1}{2}\left(\mathcal{C}+n_{2}+2-n_{2} \rho\right) x_{1}+\frac{1}{2} n_{2} x_{2}+G_{1}(\rho)
$$

and

$$
H\left(x_{2}, \rho\right)=-\frac{1}{2}\left(\mathcal{C}+n_{2}+\left(4-n_{2}\right) \rho\right) x_{2}+H_{1}(\rho)
$$

and $F(\rho), H_{1}(\rho), G_{1}(\rho)$ satisfy the ODE system

$$
\begin{aligned}
F^{\prime} & =-\frac{1}{\rho(1-\rho)}\left(\frac{1}{2}\left(\mathcal{C}+n_{2}+\left(4-n_{2}\right) \rho\right) F+2 H_{1}\right), \\
H_{1}^{\prime} & =\frac{1}{2}\left(n_{2}-2\right) F-G_{1}, \\
G_{1}^{\prime} & =0 .
\end{aligned}
$$

Remark 6.1. Formulas (6.5) and (6.7) are exactly the normal forms obtained in [9] for compatible pairs $h$ and $L$ in the case when $L$ is conjugate to a $2 \times 2$ resp. $3 \times 3$ Jordan block with a non-constant eigenvalue. Notice that these formulas are only meaningful at those points where $F+x \neq 0$ (resp. $\left.F+2 x_{2} \neq 0\right)$.

Remark 6.2. In part (1) of Corollary 6.2, we actually re-derived the formulas (5.12) for the components of $v$ and the functions $F_{i}$ parametrizing the metric

$$
h=\left.g\right|_{\mathcal{L}}=\sum_{i=1}^{\ell} \frac{\Delta_{i}}{F_{i}} \mathrm{~d} \rho_{i}^{2}
$$

obtained from the metric $g$ by restricting it to leaves $\mathcal{L}$ of the distribution $\mathcal{U}$. 
Proof of Corollary 6.2. Theorem 6.1 shows that $g_{1}, L_{1}$ and $v_{1}$ have to satisfy the equations

$$
\mathcal{L}_{v_{1}} L_{1}=-L_{1}^{2}+L_{1} \text { and } \mathcal{L}_{v_{1}} g_{1}=\left(n_{2}-1\right) h_{1} L_{1}-\left(\operatorname{tr} L_{1}+\mathcal{C}+n_{2}\right) g_{1},
$$

where $n_{2}=n-n_{1}$ is the dimension of the block $\left(g_{2}, L_{2}\right)$ of $(g, L)$ complementary to $\left(g_{1}, L_{1}\right)$.

(1) From the first equation in (6.9), it follows immediately that $v=\rho(1-\rho) \partial_{\rho}$. This solves the first equation identically. It is straightforward to check that the second equation in (6.9) with $n_{2}$ replaced by $n-1$ is equivalent to the ODE

$$
\frac{\mathrm{d} F}{F}=\frac{n+1+\mathcal{C}-(n+1) \rho}{\rho(1-\rho)} \mathrm{d} \rho .
$$

The solution to this ODE is (6.4) as we claimed.

(2) Since the first equation in (6.9) implies that $v_{1}$ preserves invariant subspaces of $L_{1}$, we can suppose that

$$
v_{1}=G(x, \rho) \partial_{x}+\rho(1-\rho) \partial_{\rho}
$$

for a certain function $G(x, \rho)$. Using this, together with the explicit formulas for $h_{1}$ and $L_{1}$, we see that the first equation in (6.9) is equivalent to

$$
\rho(1-\rho) F^{\prime}+G-(F+x) \partial_{x} G=0
$$

whilst the second equation in (6.9) is equivalent to the equations

$$
\rho(1-\rho) F^{\prime}+G+(F+x)\left(\partial_{x} G+1+\mathcal{C}+n_{2}+\left(1-n_{2}\right) \rho\right)=0
$$

and

$$
2 \partial_{\rho} G=\left(n_{2}-1\right)(F+x) .
$$

Substracting (6.10) from (6.11) and dividing by $F+x$ yields

$$
2 \partial_{x} G+1+\mathcal{C}+n_{2}+\left(1-n_{2}\right) \rho=0 .
$$

This shows that $G$ must be of the form

$$
G(x, \rho)=\frac{1}{2}\left(\left(n_{2}-1\right) \rho-1-\mathcal{C}-n_{2}\right) x+G_{1}(\rho)
$$

as we claimed. Inserting this into (6.10) (now equivalent to (6.11)) and (6.12), we obtain (6.6) after rearranging terms.

(3) Again, since $v_{1}$ preserves invariant subspaces of $L_{1}$, we have

$$
v_{1}=G\left(x_{1}, x_{2}, \rho\right) \frac{\partial}{\partial x_{1}}+H\left(x_{2}, \rho\right) \frac{\partial}{\partial x_{2}}+\rho(1-\rho) \frac{\partial}{\partial \rho}
$$

for certain functions $G\left(x_{1}, x_{2}, \rho\right)$ and $H\left(x_{2}, \rho\right)$. A straightforward calculation gives that the first equation in (6.9) is equivalent to the equations

$$
\begin{gathered}
-1+2 \rho+\partial_{x_{2}} H-\partial_{x_{1}} G=0 \\
2 x_{2}+F+G+\partial_{\rho} H-\left(F+2 x_{2}\right) \partial_{x_{2}} G-x_{1} \partial_{x_{1}} G=0
\end{gathered}
$$

and

$$
2 H+\rho(1-\rho) F^{\prime}-\left(F+2 x_{2}\right) \partial_{x_{2}} H=0
$$

whilst the second equation in (6.9) is equivalent to the equations

$$
\begin{gathered}
2 H+\rho(1-\rho) F^{\prime}+\left(F+2 x_{2}\right)\left(1+\mathcal{C}+n_{2}+\left(2-n_{2}\right) \rho+\partial_{x_{1}} G\right)=0, \\
\mathcal{C}+n_{2}+\left(4-n_{2}\right) \rho+2 \partial_{x_{2}} H=0, \\
\left(1+\mathcal{C}+n_{2}+\left(2-n_{2}\right) \rho+\partial_{x_{2}} H\right) x_{1}+G+\partial_{\rho} H-\left(F+2 x_{2}\right)\left(n_{2}-1-\partial_{x_{2}} G\right)=0
\end{gathered}
$$

and

$$
\left(2+\mathcal{C}+n_{2}-n_{2} \rho\right) x_{1}^{2}+2\left(G+\partial_{\rho} H\right) x_{1}-2\left(F+2 x_{2}\right)\left(\left(n_{2}-1\right) x_{1}-\partial_{\rho} G\right)=0 .
$$


Subtracting (6.16) from (6.17) and dividing by $F+2 x_{2}$, we obtain

$$
1+\mathcal{C}+n_{2}+\left(2-n_{2}\right) \rho+\partial_{x_{1}} G+\partial_{x_{2}} H=0 .
$$

Hence, using (6.14),

$$
\partial_{x_{1}} G=\frac{1}{2}\left(n_{2} \rho-2-\mathcal{C}-n_{2}\right) \text { and } \partial_{x_{2}} H=\frac{1}{2}\left(\left(n_{2}-4\right) \rho-\mathcal{C}-n_{2}\right)
$$

or, in other words,

$G\left(x_{1}, x_{2}, \rho\right)=\frac{1}{2}\left(n_{2} \rho-2-\mathcal{C}-n_{2}\right) x_{1}+\tilde{G}\left(x_{2}, \rho\right)$ and $H\left(x_{2}, \rho\right)=\frac{1}{2}\left(\left(n_{2}-4\right) \rho-\mathcal{C}-n_{2}\right) x_{2}+H_{1}(\rho)$

for certain functions $\tilde{G}\left(x_{2}, \rho\right), H_{1}(\rho)$. Inserting this back into our PDE system (6.14)-(6.20) we obtain that $(6.9)$ is equivalent to the equations

and

$$
\begin{gathered}
\frac{1}{2} n_{2} x_{2}+F+\tilde{G}+H_{1}^{\prime}-\left(F+2 x_{2}\right) \partial_{x_{2}} \tilde{G}=0, \\
\left(\mathcal{C}+n_{2}+\left(4-n_{2}\right) \rho\right) F+4 H_{1}+2 \rho(1-\rho) F^{\prime}=0, \\
-\frac{3}{2} n_{2} x_{2}+\tilde{G}+H_{1}^{\prime}+\left(F+2 x_{2}\right) \partial_{x_{2}} \tilde{G}+\left(1-n_{2}\right) F=0
\end{gathered}
$$

Substracting (6.21) from (6.23) and dividing by $F+2 x_{2}$ gives $-n_{2}+2 \partial_{x_{2}} \tilde{G}=0$ and we see that

$$
\tilde{G}\left(x_{2}, \rho\right)=\frac{1}{2} n_{2} x_{2}+G_{1}(\rho) \text {. }
$$

Inserting this formula for $\tilde{G}$ into $(6.21)-(6.24)$, we obtain that (6.9) is equivalent to the equations

$$
\begin{gathered}
\frac{1}{2}\left(2-n_{2}\right) F+G_{1}+H_{1}^{\prime}=0, \\
\left(\mathcal{C}+n_{2}+\left(4-n_{2}\right) \rho\right) F+4 H_{1}+2 \rho(1-\rho) F^{\prime}=0 \\
4 x_{2} G_{1}^{\prime}+\left(\left(2-n_{2}\right) x_{1}+2 G_{1}^{\prime}\right) F+2 x_{1}\left(G_{1}+H_{1}^{\prime}\right)=0 .
\end{gathered}
$$

The first two of these equations give the first two equations in (6.8). Multiplying the first equation by $2 x_{1}$ and subtracting it from the third gives $2\left(F+2 x_{2}\right) G_{1}^{\prime}=0$, hence, $G_{1}^{\prime}=0$ as we claimed.

Now we are ready to describe the local structure of $g, A$ and $v$ in the case when $A$ "contains" a $2 \times 2$ or $3 \times 3$ Jordan block.

Proposition 6.3. (1) Let $p \in M^{0}$ be a regular point and A contain a $2 \times 2$ Jordan block with a non-constant eigenvalue $\rho=\rho_{1}$. Then in a neighborhood of $p$ there exists a local coordinate system $x, \rho_{1}, \ldots, \rho_{\ell}, y_{1}, \ldots, y_{N}, N=m_{0}+m_{1}$, in which $g$ and $A$ take the following form:

$$
A=\left(\begin{array}{cc}
L(x, \vec{\rho}) & 0 \\
0 & A_{\mathrm{c}}(y)
\end{array}\right) \quad \text { and } \quad g=\left(\begin{array}{cc}
h(x, \vec{\rho}) & 0 \\
0 & g_{\mathrm{c}}(y) \cdot \chi_{L}\left(A_{\mathrm{c}}(y)\right)
\end{array}\right)
$$

where

$$
L=\left(\begin{array}{cccc}
L_{1}\left(x, \rho_{1}\right) & 0 & \ldots & 0 \\
0 & \rho_{2} & \ldots & 0 \\
\vdots & \vdots & \ddots & \vdots \\
0 & 0 & \ldots & \rho_{\ell}
\end{array}\right), \quad h=\left(\begin{array}{cccc}
h_{1}\left(x, \rho_{1}\right) \cdot \Delta_{1}\left(L_{1}\right) & 0 & \ldots & 0 \\
0 & F_{2}^{-1}\left(\rho_{2}\right) \cdot \Delta_{2} & \ldots & 0 \\
\vdots & \vdots & \ddots & \vdots \\
0 & 0 & \ldots F_{\ell}^{-1}\left(\rho_{\ell}\right) \cdot \Delta_{\ell}
\end{array}\right),
$$

and the ingredients in these matrices are as follows:

- $L_{1}$ and $h_{1}$ are defined by (6.5) with $\rho=\rho_{1}$,

- $\Delta_{1}(\cdot)$ is the polynomial of the form $\Delta_{1}(t)=\Pi_{j=2}^{\ell}\left(t-\rho_{j}\right)$,

- $F_{i}\left(\rho_{i}\right)=a_{i}\left(1-\rho_{i}\right)^{-\mathcal{C}} \rho_{i}^{\ell+2+\mathcal{C}}$,

- $\Delta_{i}=\left(\rho_{i}-\rho_{1}\right)^{2} \Pi_{j=2, j \neq i}^{\ell}\left(\rho_{i}-\rho_{j}\right), i=2, \ldots, \ell$, 
- $A_{\mathrm{c}}(y)$ is selfadjoint and parallel w.r.t. $g_{\mathrm{c}}(y)$.

Furthermore,

$$
v=G\left(x, \rho_{1}\right) \frac{\partial}{\partial x}+\sum_{i=1}^{\ell} \rho_{i}\left(1-\rho_{i}\right) \frac{\partial}{\partial \rho_{i}}+\ldots
$$

with $G\left(x, \rho_{1}\right)$ as in Corollary 6.2 (2), with $n_{2}=\ell-1, \rho_{1}=\rho$.

(2) Similarly, let $p \in M^{0}$ be a regular point and $A$ contain $a 3 \times 3$ Jordan block with a nonconstant eigenvalue $\rho=\rho_{1}$. Then in a neighbourhood of $p \in M^{0}$ we can choose local coordinates $x_{1}, x_{2}, \rho_{1}, \ldots, \rho_{\ell}, y_{1} \ldots, y_{N}$ such that $A$ and $g$ are given by (6.25), (6.26) where $x=\left(x_{1}, x_{2}\right)$ and the other ingredients are as follows:

- the $3 \times 3$ blocks $L_{1}\left(x_{1}, x_{2}, \rho_{1}\right)$ and $h_{1}\left(x_{1}, x_{2}, \rho_{1}\right)$ are defined by $(6.7)$ with $\rho_{1}=\rho$,

- $\Delta_{1}(\cdot)$ is the polynomial of the form $\Delta_{1}(t)=\Pi_{j=2}^{\ell}\left(t-\rho_{j}\right)$,

- $F_{i}\left(\rho_{i}\right)=a_{i}\left(1-\rho_{i}\right)^{-\mathcal{C}} \rho_{i}^{\ell+3+\mathcal{C}}, i=2, \ldots, \ell$,

- $\Delta_{i}=\left(\rho_{i}-\rho_{1}\right)^{3} \Pi_{j=2, j \neq i}^{\ell}\left(\rho_{i}-\rho_{j}\right), i=2, \ldots, \ell$,

- $A_{\mathrm{c}}(y)$ is selfadjoint and parallel w.r.t. $g_{\mathrm{c}}(y)$.

Furthermore,

$$
v=G\left(x_{1}, x_{2}, \rho_{1}\right) \frac{\partial}{\partial x_{1}}+H\left(x_{2}, \rho_{1}\right) \frac{\partial}{\partial x_{2}}+\sum_{i=1}^{\ell} \rho_{i}\left(1-\rho_{i}\right) \frac{\partial}{\partial \rho_{i}}+\ldots
$$

with $G\left(x_{1}, x_{2}, \rho_{1}\right)$ and $H\left(x_{2}, \rho\right)$ as in Corollary 6.2 (3), with $n_{2}=\ell-1, \rho_{1}=\rho$.

Proof. The formulas (6.25) and (6.26) (with $F(\rho), F_{2}\left(\rho_{2}\right), \ldots, F_{\ell}\left(\rho_{\ell}\right)$ being arbitrary functional parameters) are just a reformulation of the main result of [9] in the case when $A$ has the algebraic type described above.

In our situation we have, in addition, a projective vector field $v$ satisfying (6.1). Consider the natural decomposition of $v$ that corresponds to the splitting (6.25) of $g, A$ into "constant" and "non-constant" blocks: $v=v_{\text {nc }}(x, \vec{\rho})+v_{\mathrm{c}}(y)$.

It is easy to see (cf. (5.13), (5.14)) that (6.1) can be rewritten for the non-constant block without any change, i.e.,

$$
\mathcal{L}_{v_{\mathrm{nc}}} L=-L^{2}+L, \quad \mathcal{L}_{v_{\mathrm{nc}}} h=-h L-(\operatorname{tr} L+\mathcal{C}) h .
$$

Here $\operatorname{tr} A+\mathcal{C}^{\prime}=\operatorname{tr} L+\mathcal{C}$ and $\mathcal{C}=\mathcal{C}^{\prime}+m_{1}$ where $m_{1}$ is the multiplicity of the constant eigenvalue 1 or, which is the same, $m_{1}=\operatorname{tr} A_{\mathrm{c}}$.

After this remark, Proposition 6.3 follows immediately by applying Theorem 6.1 and Corollary 6.2 (for $h, L$ and $v_{\text {nc }}$ but not $v$ !) to reconstruct the functions $F(\rho), F_{2}\left(\rho_{2}\right), \ldots, F_{\ell}\left(\rho_{\ell}\right)$ as well as the components of $v_{\text {nc }}$ (the components of $v_{\mathrm{c}}(y)$ are not important for our purposes and we ignore them, in Proposition 6.3 they are denoted by ...).

Partitioning local coordinates into two groups $x, \rho_{1}, \ldots, \rho_{\ell}$ and $y_{1}, \ldots, y_{N}$ determines two natural integrable distributions $\mathcal{U}$ and $\mathcal{U}^{\perp}$ on $M^{0}$ similar to those from $\S 5.5$. All geometric properties of the corresponding foliations listed in Proposition 5.6 still hold with one little amendment that $\operatorname{dim} \mathcal{U}=\ell+1$ or $\ell+2$ (but not $\ell$ as before) so that now we should think of $x$ as an additional coordinate to $\rho_{i}$ 's.

The next statement is an analogue of Proposition 5.10. Consider the domain $U \subset \mathbb{R}^{\ell+1}\left(x, \rho_{1}, \ldots, \rho_{\ell}\right)$ in the case of a $2 \times 2$ Jordan block (resp. $U \subset \mathbb{R}^{\ell+2}\left(x_{1}, x_{2}, \rho_{1}, \ldots, \rho_{\ell}\right)$ in the case of a $3 \times 3$ Jordan block) on which the above local formulas (6.26) for $h$ are naturally defined. More precisely, $U$ is defined by the inequalities (6.2) for the $\rho_{i}$ 's and the additional coordinates $x$ and $x_{2}$ satisfy $F\left(\rho_{1}\right)+x \neq 0$ for a $2 \times 2$-block and resp. $F_{1}\left(\rho_{1}\right)+2 x_{2} \neq 0$ for a $3 \times 3$ block, see Remark 6.1.

Proposition 6.4. There is a natural isometric immersion $\phi: U \rightarrow M$ (as a leaf of the totally geodesic foliation determined by $\mathcal{U})$. In other words, the above formulas (6.25) and (6.26) have global meaning on $M$ for all admissible values of coordinates. 
Proof. The proof is almost identical to that of Proposition 5.10. We start with a certain point $p \in M^{0}$ and locally identify the leaf of $\mathcal{U}$ through $p$ with $U$ by using a canonical coordinate system in its neighbourhood constructed in Proposition 6.3.

After this we use prolongation along a path as in Proposition 5.10. The only thing we need to explain is why such a prolongation is always possible. More specifically, we need to show that the limit point of the curve $\phi(a(t))$ as $t \rightarrow T_{0}$ (we use the same notation as in Proposition 5.10) cannot leave $M^{0}$, the set of regular points.

Since, $\phi$ preserves the eigenvalues of $L$, the multiplicities of the eigenvalues remain unchanged and the inequalities (6.2) hold at the limit point. The condition $\mathrm{d} \rho_{i}=0$ is fulfilled automatically and we only need to check that the Jordan block "survives" at the limit point. A priori under continuous deformations the Jordan block may split into smaller blocks and we need to show that this event may not happen under our assumptions.

To prove this fact we use the following algebraic lemma.

Lemma 6.5. Let $h$ be a non-degenerate bilinear form of Lorentzian signature and $L$ be an $h$ selfadjoint endomorphism. Assume that $L$ has a single real eigenvalue $\rho$ and $e_{1}$ is a $\rho$-eigenvector of $L$. Then in dimension 2 and 3 we have respectively:

(1) For any canonical basis $e_{1}, e_{2}$ (i.e., such that $h=\left(\begin{array}{ll}0 & 1 \\ 1 & 0\end{array}\right)$ or, equivalently, $h\left(e_{i}, e_{j}\right)=$ $\left.\delta_{i, 3-j}\right)$, the matrix of $L$ has the form

$$
L=\left(\begin{array}{cc}
\rho & \alpha \\
0 & \rho
\end{array}\right) .
$$

Moreover, $\alpha$ does not depend on the choice of $e_{2}$, and can be computed from the following formula $\alpha=\operatorname{vol}_{h}\left(e_{2},(L-\rho \mathrm{Id}) e_{2}\right)$.

(2) For any canonical basis $e_{1}, e_{2}, e_{3}$ (i.e., such that $h=\left(\begin{array}{lll}0 & 0 & 1 \\ 0 & 1 & 0 \\ 1 & 0 & 0\end{array}\right)$ or, equivalently, $h\left(e_{i}, e_{j}\right)=$ $\left.\delta_{i, 4-j}\right)$, the matrix of $L$ has the form

$$
L=\left(\begin{array}{ccc}
\rho & \alpha & \beta \\
0 & \rho & \alpha \\
0 & 0 & \rho
\end{array}\right)
$$

Moreover, $\alpha$ does not depend on the choice of $e_{2}$ and $e_{3}$, and can be computed from the following formula $\alpha=\operatorname{vol}_{h}\left(e_{3},(L-\rho \mathrm{Id}) e_{3},(L-\rho \mathrm{Id})^{2} e_{3}\right)^{\frac{1}{3}}$.

Proof. The proof is straightforward and we only give some comments for dim $=3$. The first statement follows immediately from two facts:

(1) $L$ is $h$-selfadjoint and therefore the matrices of $L$ and $h$ satisfy $L^{\top} h=h L$;

(2) the first column of $L$ is $(\rho, 0,0)^{\top}$.

The formula for $\alpha$ is obvious in the basis $e_{1}, e_{2}, e_{3}$. We now check that this formula is independent on the choice of $e_{2}$ and $e_{3}$. Let $e_{1}, e_{2}^{\prime}, e_{3}^{\prime}$ be another canonical basis. Then $e_{3}^{\prime}=$ $a_{1} e_{1}+a_{2} e_{2}+a_{3} e_{3}$, but $h\left(e_{1}, e_{3}\right)=h\left(e_{1}, e_{3}^{\prime}\right)=1$ immediately implies that $a_{3}=1$. Hence, using the explicit formula for $L$, we can easily conclude that the additional terms $a_{1} e_{1}+a_{2} e_{2}$ do not contribute. Indeed,

$$
\begin{aligned}
& \operatorname{vol}_{h}\left(e_{3}^{\prime},(L-\rho \mathrm{Id}) e_{3}^{\prime},(L-\rho \mathrm{Id})^{2} e_{3}^{\prime}\right)= \\
& \operatorname{vol}_{h}\left(e_{3}+a_{1} e_{1}+a_{2} e_{2},(L-\rho \mathrm{Id}) e_{3}+a_{2}(L-\rho \mathrm{Id}) e_{2},(L-\rho \mathrm{Id})^{2} e_{3}\right)= \\
& \operatorname{vol}_{h}\left(e_{3}+a_{1} e_{1}+a_{2} e_{2}, \beta e_{1}+\alpha e_{2}, \alpha^{2} e_{1}\right)=\alpha^{3} \operatorname{vol}_{g}\left(e_{3}, e_{2}, e_{1}\right)=\alpha^{3} .
\end{aligned}
$$

This lemma gives us a simple method to recognise if $L$ has a Jordan block of maximal size or not.

To verify that the limit point $p$ of the curve $\phi(a(t))$, as $t \rightarrow T_{0}$, is regular, i.e., the Jordan block "survives", we will use the fact that the eigenvalue $\rho_{1}$ of the Jordan block is a smooth function 
on $M^{0}$. Moreover, the vector field $e_{1}=\operatorname{grad} \rho_{1}$ does not vanish and is an eigenvector of the $\rho_{1}$-block. Notice that these conditions hold not only on $M^{0}$, but also on a slightly bigger set $\widetilde{M}^{0}$ $\left(M^{0} \subset \widetilde{M^{0}}\right)$ which can be characterised by the property that the multiplicities of eigenvalues are fixed but the algebraic type of $L$ is allowed to change, namely, the Jordan $\rho_{1}$-block may split into smaller $\rho_{1}$-blocks. Notice that the natural splitting into blocks corresponding to the eigenvalues of $g$ makes sense on $\widetilde{M}^{0}$, so we can work with each block separately. An important additional fact, we are going to use, is that $\phi$, by construction, preserves grad $\rho_{1}$.

We use Lemma 6.5 to verify that the parameter $\alpha$ in the matrix of $L(p)$ does not vanish. Since both $L$ and $e_{1}$ are smooth, we have by continuity $\alpha=\lim _{t \rightarrow T_{0}} \alpha(t)$, where $\alpha(t)$ is computed at the point $\phi(a(t))$ (w.r.t. to $e_{1}=\operatorname{grad} \rho$ ). But since $\phi$ is an isometry whenever it is well defined, then the limit can be computed on $U$. Since all the points of $U$ are regular by construction, we have $\lim _{t \rightarrow T_{0}} \alpha(t) \neq 0$ as required.

Thus the limit point of $\phi(a(t)), t \rightarrow T_{0}$, is regular and the further continuation of $\phi$ is possible which completes the proof.

Now to prove that Jordan blocks with non-constant eigenvalues cannot appear on compact manifolds, we compute one special eigenvalue of the curvature operator of the metric $g$ given by the formulas from Proposition 6.3. For this computation we use the following real analogue of Proposition A.2, which can be proved in a similar way.

Proposition 6.6 ([5]). Let $g$ and $L$ be compatible in the projective sense and $\Lambda$ be as in (1.7). Let $\nabla \Lambda=f(L)$ at some point $p \in M$, where $f(\cdot)$ is a polynomial (or, more generally, an analytic function) and suppose $L(p)$ has a non-trivial Jordan $\rho$-block. Then one of the eigenvalues of the curvature operator of $g$ at the point $p$ takes the form

$$
f^{\prime}(\rho) \text {. }
$$

This number can be computed for our metric $g$ (equivalently, for $h$ given by (6.26)) A straightforward calculation shows the following:

Proposition 6.7. (1) For a $2 \times 2$ Jordan $\rho_{1}$-block, we have

$$
f^{\prime}\left(\rho_{1}\right)=-\frac{F_{1}^{\prime}\left(\rho_{1}\right)}{\left(F_{1}\left(\rho_{1}\right)+x\right)^{3} \prod_{i \geq 2}\left(\rho_{1}-\rho_{i}\right)}+\sum_{i \geq 2} \frac{F_{i}\left(\rho_{i}\right)}{4\left(\rho_{i}-\rho_{1}\right)^{4} \prod_{j \notin\{1, i\}}\left(\rho_{i}-\rho_{j}\right)} .
$$

(2) For a $3 \times 3$ Jordan $\rho_{1}$-block, we have

$$
f^{\prime}\left(\rho_{1}\right)=-\frac{3}{4\left(F_{1}\left(\rho_{1}\right)+2 x_{2}\right)^{2} \prod_{i \geq 2}\left(\rho_{1}-\rho_{i}\right)}+\sum_{i \geq 2} \frac{F_{i}\left(\rho_{i}\right)}{4\left(\rho_{i}-\rho_{1}\right)^{5} \prod_{j \notin\{1, i\}}\left(\rho_{i}-\rho_{j}\right)} .
$$

These formulas immediately imply that the quantity $f^{\prime}\left(\rho_{1}\right)$ (which is some special eigenvalue of the curvature operator of $g$ ) is unbounded on $M^{0}$. Indeed, $x$ and $x_{2}$ may vary independently of the other coordinates and, in particular, we may fix the values of all $\rho_{i}$ 's and then vary $x\left(\right.$ resp. $\left.x_{2}\right)$ so that $F_{1}\left(\rho_{1}\right)+x$ (resp. $\left.F_{1}\left(\rho_{1}\right)+2 x_{2}\right)$ tends to 0 and therefore $f^{\prime}\left(\rho_{1}\right) \rightarrow \infty$, which is impossible due to compactness of $M$. Thus, Jordan blocks with non-constant eigenvalues may not occur in our situation and this conclusion completes the proof of Theorem 1.2.

\section{Appendix A. Eigenvalues of the Curvature operator}

In what follows, we consider a real vector space $V$ with a complex structure $J$ and an inner product $g$ (not necessarily positive definite) such that $g(J u, J v)=g(u, v)$. Such a triple $(V, g, J)$ will be referred to as a pseudo-hermitian vector space. We use the symbol

$$
\mathfrak{u}(g, J)=\{X \in \mathfrak{g l}(V):[X, J]=0 \text { and } g(X u, v)=-g(u, X v)\}
$$

to denote the space (Lie algebra) of skew-hermitian endomorphisms on $V$.

Let us first reformulate the integrability condition for equation (1.4) in a way adapted to the Lie theory. Recall that the Riemann curvature operator (at a point $x \in M$ ) can be understood as a map $R: T_{x} M \otimes T_{x} M \rightarrow \mathfrak{s o}(g), R(u, v)=\nabla_{u} \nabla_{v}-\nabla_{v} \nabla_{u}-\nabla_{[u, v]}$. Taking into account the fact that we are dealing with a Kähler manifold and using the symmetries of the curvature tensor of a 
Kähler metric, we can also think of $R$ as an operator defined on the unitary Lie algebra (we still use the same notation)

$$
R: \mathfrak{u}(g, J) \rightarrow \mathfrak{u}(g, J)
$$

by setting $R(u, v)=\frac{1}{4} R\left(u \wedge_{J} v\right)$, where

$$
u \wedge_{J} v=u^{b} \otimes v-v^{b} \otimes u+(J u)^{b} \otimes J v-(J v)^{b} \otimes J u \in \mathfrak{u}(g, J)
$$

and $u^{b}=g(u, \cdot)$ denotes the metric dual of $u$.

Lemma A.1. Let $(M, g, J)$ be a Kähler manifold of arbitrary signature, $A \in \mathcal{A}(g, J)$ be a hermitian solution of (1.4) and $\Lambda=\frac{1}{4} \operatorname{grad}(\operatorname{tr} A)$. Then the curvature operator $R: \mathfrak{u}(g, J) \rightarrow \mathfrak{u}(g, J)$ satisfies the relation

$$
[R(X), A]=4[X, \nabla \Lambda] \text { for all } X \in \mathfrak{u}(g, J) .
$$

Proof. The Ricci identity applied to an arbitrary field of endomorphisms $A$ reads

$$
\nabla_{u} \nabla_{v} A-\nabla_{v} \nabla_{u} A-\nabla_{[u, v]} A=[R(u, v), A]
$$

for any vector fields $u, v$. Let now $A \in \mathcal{A}(g, J)$ be a hermitian solution of (1.4). Since $\nabla \Lambda$ is $g$-selfadjoint and $J$-linear, i.e., hermitian too, we have

$$
\begin{gathered}
\nabla_{u} \nabla_{v} A-\nabla_{v} \nabla_{u} A-\nabla_{[u, v]} A \\
=v^{b} \otimes \nabla_{u} \Lambda+\left(\nabla_{u} \Lambda\right)^{b} \otimes v-u^{b} \otimes \nabla_{v} \Lambda-\left(\nabla_{v} \Lambda\right)^{b} \otimes u \\
+(J v)^{b} \otimes \nabla_{J u} \Lambda+\left(\nabla_{J u} \Lambda\right)^{b} \otimes J v-(J u)^{b} \otimes \nabla_{J v} \Lambda-\left(\nabla_{J v} \Lambda\right)^{b} \otimes J u \\
=[X, \nabla \Lambda],
\end{gathered}
$$

where $X=u \wedge_{J} v$. This proves formula (A.2) for elements $X \in \mathfrak{u}(g, J)$ of the form $u \wedge_{J} v$ and the claim follows from the fact that all skew-hermitian endomorphisms are sums of such elements.

Remark A.1. If formula (A.2) holds for an operator $R: \mathfrak{u}(g, J) \rightarrow \mathfrak{u}(g, J)$ and hermitian endomorphisms $A$ and $\nabla \Lambda$ then, in fact, $\nabla \Lambda$ can be presented in the form $\nabla \Lambda=p(A)$ for some polynomial $p(\cdot)$ with real coefficients. To show this, take an arbitrary $J$-complex matrix $Y$ and consider the following algebraic relations:

$$
\operatorname{tr}(X \cdot[\nabla \Lambda, Y])=\operatorname{tr}(Y \cdot[X, \nabla \Lambda])=\frac{1}{4} \operatorname{tr}(Y \cdot[R(X), A])=\frac{1}{4} \operatorname{tr}(R(X) \cdot[A, Y]),
$$

where $X \in \mathfrak{u}(g, J)$ and $\operatorname{tr}$ denotes the complex trace. Since $\mathfrak{u}(g, J)$ spans $\mathfrak{g l}\left(T_{x} M, J\right)$ in the complex sense, we conclude that $[\nabla \Lambda, Y]=0$ for any $Y$ commuting with $A$. It is a well-known algebraic fact that in this case $\nabla \Lambda$ can be written as a polynomial of $A$. Moreover, as both $A$ and $\nabla \Lambda$ are hermitian, this polynomial must be real, i.e. with real coefficients.

Proposition A.2 below together with formula (A.2) allows us to calculate eigenvalues of the curvature operator in terms of the eigenvalues of $A$ and $\nabla \Lambda$. For the main concepts of the proof of this proposition and for the relation to sectional operators in the theory of integrable systems compare also with $[6, \S 3]$ and $[5,12]$.

Proposition A.2. Let $(V, g, J)$ be a pseudo-hermitian vector space and let $A: V \rightarrow V$ be a hermitian endomorphism. Suppose an operator $R: \mathfrak{u}(g, J) \rightarrow \mathfrak{u}(g, J)$ satisfies

$$
[R(X), A]=[X, B] \text { for all } X \in \mathfrak{u}(g, J),
$$

where $B=p(A)$ and $p(\cdot)$ is a polynomial with real coefficients. Then we have the following:

(1) For all real eigenvalues $\lambda_{i} \neq \lambda_{j}$ of $A$,

$$
\frac{p\left(\lambda_{i}\right)-p\left(\lambda_{j}\right)}{\lambda_{i}-\lambda_{j}}
$$

is an eigenvalue of $R$.

(2) If $A$ has a non-trivial $\lambda_{i}$-Jordan block, $\lambda_{i} \in \mathbb{R}$, then $p^{\prime}\left(\lambda_{i}\right)$ is an eigenvalue of $R$. 
Remark A.2. The first item of Proposition A.2 can be understood in a slightly different way. Notice that $B=p(A)$ implies that each eigenvector of $A$ with an eigenvalue $\lambda_{i}$ is, at the same time, an eigenvector of $B$ with the eigenvalue $m_{i}=p\left(\lambda_{i}\right)$. Hence the formula for the eigenvalue of $R$ from item (1) can be rewritten as $\frac{m_{i}-m_{j}}{\lambda_{i}-\lambda_{j}}$ so that we do not actually need to find $p(\cdot)$ explicitly; it is sufficient to know the eigenvalues $m_{i}$ of $B$ corresponding to $\lambda_{i}$.

The second item of Proposition A.2 can also be modified by using the following simple fact from Linear Algebra. Let $\lambda$ be an eigenvalue of an endomorphism $A$ having a non-trivial $\lambda$-Jordan block. Let $p(\cdot)$ and $q(\cdot)$ be two polynomials (or even more generally, analytic functions) such that $p(A)=q(A)$, then $p(\lambda)=q(\lambda)$ and $p^{\prime}(\lambda)=q^{\prime}(\lambda)$. It follows from this statement that the polynomial $p$ in the second item of Proposition A.2 can be replaced by any other function $q$ satisfying $p\left(\left.A\right|_{V_{\lambda_{i}}}\right)=q\left(\left.A\right|_{V_{\lambda_{i}}}\right)$, where $V_{\lambda_{i}}$ denotes the generalised $\lambda_{i}$-eigenspace of $A$.

Proof of Proposition A.2. We start with some general considerations regarding formula (A.3). We view this formula as an equation on $R$ for fixed $A$ and $B=p(A)$. Suppose $R_{1}, R_{2}: \mathfrak{u}(g, J) \rightarrow$ $\mathfrak{u}(g, J)$ are two solutions of (A.3). Then,

$$
\left[R_{1}(X)-R_{2}(X), A\right]=0 \text { for all } X \in \mathfrak{u}(g, J),
$$

that is, $R_{1}-R_{2}$ takes values in the Lie algebra

$$
\mathfrak{g}_{A}=\{X \in \mathfrak{u}(g, J):[X, A]=0\},
$$

the centraliser of $A$ in $\mathfrak{u}(g, J)$. Thus, any solution $R$ of (A.3) is unique up to adding an operator $\mathfrak{u}(g, J) \rightarrow \mathfrak{g}_{A}$. Moreover, an operator $R$ satisfying (A.3) preserves the centraliser $\mathfrak{g}_{A}$. Indeed, since $B=p(A)$ is a polynomial in $A$, we have $[X, B]=0$ for all $X \in \mathfrak{g}_{A}$. Then (A.3) implies $[R(X), A]=0$ for $X \in \mathfrak{g}_{A}$, showing

$$
R\left(\mathfrak{g}_{A}\right) \subseteq \mathfrak{g}_{A}
$$

as we claimed. For any solution $R$ of (A.3), we may therefore consider the induced operator

$$
\tilde{R}: \mathfrak{u}(g, J) / \mathfrak{g}_{A} \rightarrow \mathfrak{u}(g, J) / \mathfrak{g}_{A}
$$

on the quotient space. It is a general fact that eigenvalues of the quotient operator $\tilde{R}$ are eigenvalues of the original operator $R$. On the other hand, we have just seen that the quotient map $\tilde{R}$ is the same for all solutions $R$ of (A.3). We will use these facts by working with the quotient map $\tilde{R}_{0}$ coming from a special solution $R_{0}$ of (A.3) defined by

$$
R_{0}=\left.\frac{\mathrm{d}}{\mathrm{d} t} p(A+t X)\right|_{t=0} .
$$

This is indeed a solution of (A.3) as follows immediately from differentiating the identity $[p(A+$ $t X), A+t X]=0$ at $t=0$. By definition, if $p(t)=\sum_{k=0}^{m} a_{k} t^{k}$, then

$$
R_{0}(X)=\sum_{k=1}^{m} a_{k} \sum_{p+q=k-1} A^{p} X A^{q} .
$$

Hence for a generating element $u \wedge_{J} v$, we obtain

$$
R_{0}\left(u \wedge_{J} v\right)=\sum_{k=1}^{m} a_{k} \sum_{p+q=k-1} A^{p} u \wedge_{J} A^{q} v .
$$

We are now in the position to prove Proposition A.2. First, we show that $R_{0}$ has eigenvalues as given in part (1) and (2) of the proposition:

(1) Suppose $u$ and $v$ are eigenvectors of $A$ for real eigenvalues $\lambda_{i}$ and $\lambda_{j}$ respectively, $\lambda_{i} \neq \lambda_{j}$. Then (A.4) becomes equal to

$$
R_{0}\left(u \wedge_{J} v\right)=\left(\sum_{k=1}^{m} a_{k} \sum_{r=0}^{k-1} \lambda_{i}^{r} \lambda_{j}^{k-1-r}\right) u \wedge_{J} v=\left(\sum_{k=1}^{m} a_{k} \frac{\lambda_{i}^{k}-\lambda_{j}^{k}}{\lambda_{i}-\lambda_{j}}\right) u \wedge_{J} v=\frac{p\left(\lambda_{i}\right)-p\left(\lambda_{j}\right)}{\lambda_{i}-\lambda_{j}} u \wedge_{J} v .
$$

Hence,

$$
\frac{p\left(\lambda_{i}\right)-p\left(\lambda_{j}\right)}{\lambda_{i}-\lambda_{j}}
$$


is an eigenvalue of $R_{0}$ with eigenvector $u \wedge_{J} v$.

(2) Let us first argue, that without loss of generality we can suppose that a fixed real eigenvalue $\lambda_{i}$ of $A$ is equal to zero. Indeed, using $\tilde{A}=A-\lambda_{i} \cdot$ Id instead of $A$ in (A.3), the equation (A.3) holds for $R_{0}, \tilde{A}$ and the same $B=\tilde{p}(\tilde{A})$ for another polynomial $\tilde{p}(t)=\sum_{k=0}^{m} \tilde{a}_{k} t^{k}$ that is equal to $p\left(t+\lambda_{i}\right)$. Since $\tilde{p}^{\prime}(0)=p^{\prime}\left(\lambda_{i}\right)$, we may assume that the fixed eigenvalue $\lambda_{i}$ under consideration is equal to zero. Suppose $A$ has a non-trivial $\lambda_{i}$-Jordan block and denote by $V_{\lambda_{i}}$ the generalised $\lambda_{i}$-eigenspace. Let $u \in V_{\lambda_{i}}$ be an eigenvector of $A$, i.e. $A u=0$, and $v \in V_{\lambda_{i}}$ satisfy $A v=u$. Then (A.4) becomes

$$
R_{0}\left(u \wedge_{J} v\right)=\sum_{k=1}^{m} a_{k}\left(u \wedge_{J} A^{k-1} v\right)=a_{1} u \wedge_{J} v
$$

Thus, $a_{1}$ is an eigenvalue of $R_{0}$ with eigenvector $u \wedge_{J} v$. Since $a_{1}=p^{\prime}(0)$, the eigenvalue is as in part (2) of Proposition A.2.

To summarize, we have shown that $R_{0}$ has eigenvalues as given in part (1) and (2) of the proposition. It remains to show that these eigenvalues are also eigenvalues for the quotient map $\tilde{R}_{0}$. Since for an arbitrary operator $R$ solving (A.3) we have $\tilde{R}=\tilde{R}_{0}$, we then obtain that $\tilde{R}$ and hence $R$, has eigenvalues as in part (1) and (2) of the proposition.

It is straightforward to show that for any operator $\varphi: V \rightarrow V$ with a $\varphi$-invariant subspace $U \subseteq V$, an eigenvalue $\lambda$ of $\varphi$ is also an eigenvalue of the quotient map

$$
\tilde{\varphi}: V / U \rightarrow V / U
$$

if and only if the generalised $\lambda$-eigenspace of $\varphi$ is not contained in $U$ (although, it may have a non-trivial intersection with $U$ ).

To complete the proof of Proposition A.2, it therefore suffices to show the following statements, each of which proves one of the parts of the proposition:

(1) $u \wedge_{J} v \notin \mathfrak{g}_{A}$ for eigenvectors $u$ and $v$ of $A$ corresponding to real eigenvalues $\lambda_{i}$ and $\lambda_{j}$ respectively, $\lambda_{i} \neq \lambda_{j}$.

(2) $u \wedge_{J} v \notin \mathfrak{g}_{A}$ for an eigenvector $u$ of $A$ corresponding to a real eigenvalue $\lambda_{i}$ and a vector $v \in V_{\lambda_{i}}$ such that $A v=u+\lambda_{i} v$.

Introducing the notation

$$
u \odot_{J} v=u^{b} \otimes v+v^{b} \otimes u+(J u)^{b} \otimes J v+(J v)^{b} \otimes J u,
$$

we have $\left[u \wedge_{J} v, A\right]=A u \odot_{J} v-u \odot_{J} A v$. Thus, for case (1) we obtain

$$
\left[u \wedge_{J} v, A\right]=\left(\lambda_{i}-\lambda_{j}\right) u \odot_{J} v
$$

which is non-zero, hence, $u \wedge_{J} v \notin \mathfrak{g}_{A}$. For case (2) we obtain

$$
\left[u \wedge_{J} v, A\right]=\lambda_{i} u \odot_{J} v-u \odot_{J}\left(u+\lambda_{i} v\right)=-u \odot_{J} u
$$

which is non-zero, hence, $u \wedge_{J} v \notin \mathfrak{g}_{A}$. This finishes the proof of the proposition.

\section{REFERENCES}

[1] V. Apostolov, D. M. J. Calderbank, P. Gauduchon, Hamiltonian 2-forms in Kähler geometry. I. General theory, J. Differential Geom. 73(2006), no. 3, 359-412, MR2228318

[2] V. Apostolov, D. M. J. Calderbank, P. Gauduchon, C. W. Tønnesen-Friedman, Hamiltonian 2-forms in Kähler geometry. II. Global classification, J. Diff. Geom. 68(2004), no. 2, 277-345, MR2144249

[3] V. Apostolov, D. M. J. Calderbank, P. Gauduchon, C. W. Tønnesen-Friedman, Hamiltonian 2-forms in Kähler geometry. III. Extremal metrics and stability, Invent. Math. 173(2008), no. 3, 547-601, MR2425136

[4] V. Apostolov, D. M. J. Calderbank, P. Gauduchon, C. W. Tønnesen-Friedman, Hamiltonian 2-forms in Kähler geometry. IV. Weakly Bochner-flat Kähler manifolds, Comm. Anal. Geom. 16(2008), no. 1, 91-126, MR2411469

[5] A. V. Bolsinov, Argument shift method and sectional operators: applications to differential geometry, accepted by Fund. and Appl. Math.

[6] A. V. Bolsinov, V. Kiosak, V. S. Matveev, A Fubini theorem for pseudo-Riemannian geodesically equivalent metrics, J. Lond. Math. Soc. 80(2009), no. 2, 341-356, MR2545256

[7] A. V. Bolsinov, V. S. Matveev, Geometrical interpretation of Benenti systems, J. Geom. Phys. 44(2003), no. 4, 489-506, MR1943174

[8] A. V. Bolsinov, V. S. Matveev, Splitting and gluing lemmas for geodesically equivalent pseudo-Riemannian metrics, Trans. Amer. Math. Soc. 363(2011), no. 8, 4081-4107, MR2792981 
[9] A. V. Bolsinov, V. S. Matveev, Local normal forms for geodesically equivalent pseudo-Riemannian metrics, Trans. Amer. Math. Soc. 367(2015), no. 9, 6719-6749, MR3356952

[10] A. V. Bolsinov, V. S. Matveev, T. Mettler, S. Rosemann, Four-dimensional Kähler metrics admitting cprojective vector fields, J. Math. Pures Appl. (9) 103(2015), no. 3, 619-657.

[11] A. V. Bolsinov, V. S. Matveev, G. Pucacco, Normal forms for pseudo-Riemannian 2-dimensional metrics whose geodesic flows admit integrals quadratic in momenta, J. Geom. Phys. 59(2009), no. 7, 1048-1062, MR2536861

[12] A. V. Bolsinov, D. Tsonev, On a new class of holonomy groups in pseudo-Riemannian geometry, J. Diff. Geom. 97(2014), no. 3, 377-394, MR3233515

[13] C. Boubel, On the algebra of parallel endomorphisms of a pseudo-Riemannian metric, J. Differential Geom. 99(2015), no. 1, 77-123, MR3299823

[14] D. Calderbank, M. Eastwood, V. S. Matveev, K. Neusser, C-projective geometry, in preparation

[15] G. de Rham, Sur la réductibilité d'un espace de Riemann, Comment. Math. Helv. 26(1952), 328-344, MR0052177

[16] U. Dini, Sopra un problema che si presenta nella teoria generale delle rappresentazioni geografiche di una superficie su di un'altra, Ann. Mat., ser. 2, 3 (1869), 269-293

[17] V. V. Domashev, J. Mikes, On the theory of holomorphically projective mappings of Kählerian spaces, Mat. Zametki 23(1978), no. 2, 297-303, MR0492674

[18] M. Eastwood, V. S. Matveev, Metric connections in projective differential geometry, Symmetries and overdetermined systems of partial differential equations, 339-350, IMA Vol. Math. Appl., 144, Springer, New York, 2008, MR2384718

[19] A. Fedorova, V. Kiosak, V. S. Matveev, S. Rosemann, The only Kähler manifold with degree of mobility at least 3 is $\left(\mathbb{C} P(n), g_{\text {Fubini-Study }}\right)$, Proc. Lond. Math. Soc. (3) 105(2012), no. 1, 153-188, MR2948791

[20] N. J. Hitchin, A. Karlhede, U. Lindström, M. Roček, Hyper-Kähler metrics and supersymmetry, Comm. Math. Phys. 108(1987), no. 4, 535-589, MR0877637

[21] V. Kiosak, V. S. Matveev, Proof of the projective Lichnerowicz conjecture for pseudo-Riemannian metrics with degree of mobility greater than two, Comm. Mat. Phys. 297(2010), no. 2, 401-426.

[22] K. Kiyohara, Two classes of Riemannian manifolds whose geodesic flows are integrable, Mem. Amer. Math. Soc. 130, no. 619, viii+143 pp., 1997

[23] K. Kiyohara, P. J. Topalov, On Liouville integrability of h-projectively equivalent Kähler metrics, Proc. Amer. Math. Soc. 139(2011), 231-242.

[24] Levi-Civita, T. On the transformations of the dynamical equations, Translated from the Italian [Ann. Mat. 24 (1896), 255-300] and commented by S. Benenti. Regul. Chaotic Dyn. 14(2009), no. 4-5, 580-614.

[25] V. S. Matveev, Lichnerowicz-Obata conjecture in dimension two, Comm. Math. Helv. 80(2005), no. 3, 541-570.

[26] V. S. Matveev, Proof of the projective Lichnerowicz-Obata conjecture, J. Diff. Geom. 75(2007), no. 3, 459-502.

[27] V. S. Matveev, P. Mounoud, Gallot-Tanno theorem for closed incomplete pseudo-Riemannian manifolds and applications, Ann. Glob. Anal. Geom. 38(2010), 259-271

[28] K. Melnick, K. Neusser, Strongly essential flows on irreducible parabolic geometries, accepted by Trans. Amer. Math. Soc., arXiv: 1410.4647

[29] V.S. Matveev, Pseudo-Riemannian metrics on closed surfaces whose geodesic flows admit non-trivial integrals quadratic in momenta, and proof of the projective Obata conjecture for two-dimensional pseudo-Riemannian metrics, J. Math. Soc. Jpn. 64(2012) no. 1. pp. 107-152

[30] V. S. Matveev, S. Rosemann, Conification construction for Kaehler manifolds and its application in cprojective geometry, Adv. Math. 274(2015), 1-38, MR3318143

[31] V. S. Matveev, S. Rosemann, Proof of the Yano-Obata conjecture for h-projective transformations, J. Differential Geom. 92(2012), no. 2, 221-261, MR2998672

[32] J.Mikes, Holomorphically projective mappings and their generalizations, Geometry, 3. J. Math. Sci. (New York) 89(1998), no. 3, 1334-1353, MR1619720

[33] A. Moroianu, U. Semmelmann, Twistor forms on Kähler manifolds, Ann. Sc. Norm. Super. Pisa Cl. Sci. (5) 2(2003) 823-845,

[34] B. O'Neill, The fundamental equations of a submersion, Michigan Math. J. 13(1966) 459-469, MR0200865

[35] T. Otsuki, Y. Tashiro, On curves in Kaehlerian spaces, Math. J. Okayama Univ. 4(1954), 57-78, MR0066024

[36] H. Pedersen, Y. S. Poon, Hamiltonian constructions of Kähler-Einstein metrics and Kähler metrics of constant scalar curvature, Comm. Math. Phys. 136(1991), no. 2, 309-326, MR1096118

[37] U. Semmelmann, Conformal Killing forms on Riemannian manifolds, Habilitationschrift, Universität München (2002), arXiv:math.DG/0206117

[38] U. Semmelmann, Conformal Killing forms on Riemannian manifolds, Math. Z. 245(2003), no. 3, 503-527.

[39] N. S. Sinjukov, Geodesic mappings of Riemannian spaces, (in Russian) "Nauka", Moscow, 1979.

[40] Y. Tashiro, On a holomorphically projective correspondence in an almost complex space, Math. J. Okayama Univ. 6(1957), 147-152, MR0087181

[41] H. Wu, On the de Rham decomposition theorem, Illinois J. Math. 8(1964), 291-311, MR0161280

[42] K. Yamauchi, On infinitesimal projective transformations, Hokkaido Math. J. 3(1974), 262-270.

[43] K. Yano, Differential geometry on complex and almost complex spaces, International Series of Monographs in Pure and Applied Mathematics, 49 A Pergamon Press Book. The Macmillan Co., New York 1965 xii+326 pp. 
[44] K. Yano, H. Hiramatu, Isometry of Kaehlerian manifolds to complex projective spaces, J. Math. Soc. Japan 33(1981), no. 1, 67-78.

[45] A. Zeghib, On discrete projective transformation groups of Riemannian manifolds, arXiv:1304.6812v1

Department of Mathematical Sciences, Loughborough University, LE11 3TU, UK

E-mail address: A.Bolsinov@lboro.ac.uk

Institute of Mathematics, Friedrich-Schiller-Universität Jena, Jena, Germany.

E-mail address: vladimir.matveev@uni-jena.de

Institute of Mathematics, Friedrich-Schiller-Universität Jena, Jena, Germany.

E-mail address: stefan.rosemann@uni-jena.de 